\title{
Integrative Taxonomy and Molecular Phylogeny of the Plant-Parasitic Nematode Genus Paratylenchus (Nematoda: Paratylenchinae): Linking Species with Molecular Barcodes
}

\author{
Phougeishangbam Rolish Singh ${ }^{1, *}$, Gerrit Karssen ${ }^{1,2}$, Marjolein Couvreur ${ }^{1}$, Sergei A. Subbotin ${ }^{3,4}$ \\ and Wim Bert ${ }^{1}$ (D) \\ 1 Nematology Research Unit, Department of Biology, Ghent University, K.L. Ledeganckstraat 35, \\ 9000 Ghent, Belgium; g.karssen@nvwa.nl (G.K.); marjolein.couvreur@ugent.be (M.C.); \\ wim.bert@ugent.be (W.B.) \\ 2 National Plant Protection Organization, Wageningen Nematode Collection, P.O. Box 9102, 6700 HC \\ Wageningen, The Netherlands \\ 3 Plant Pest Diagnostic Center, California Department of Food and Agriculture, 3294 Meadowview Road, \\ Sacramento, CA 95832, USA; sergei.a.subbotin@gmail.com \\ 4 Center of Parasitology of A.N. Severtsov Institute of Ecology and Evolution of the Russian, \\ Academy of Sciences, Leninskii Prospect 33, 117071 Moscow, Russia \\ * Correspondence: PhougeishangbamRolish.Singh@Ugent.be
}

Citation: Singh, P.R.; Karssen, G.; Couvreur, M.; Subbotin, S.A.; Bert, W. Integrative Taxonomy and Molecular Phylogeny of the Plant-Parasitic Nematode Genus Paratylenchus (Nematoda: Paratylenchinae): Linking Species with Molecular Barcodes. Plants 2021, 10, 408. https://doi.org/10.3390/ plants10020408

Academic Editor: Francesc Xavier Sorribas Royo

Received: 15 January 2021

Accepted: 15 February 2021

Published: 22 February 2021

Publisher's Note: MDPI stays neutral with regard to jurisdictional claims in published maps and institutional affiliations.

Copyright: (c) 2021 by the authors. Licensee MDPI, Basel, Switzerland. This article is an open access article distributed under the terms and conditions of the Creative Commons Attribution (CC BY) license (https:/ / creativecommons.org/licenses/by/ $4.0 /)$.
Abstract: Pin nematodes of the genus Paratylenchus are obligate ectoparasites of a wide variety of plants that are distributed worldwide. In this study, individual morphologically vouchered nematode specimens of fourteen Paratylenchus species, including P. aculentus, P. elachistus, P. goodeyi, P. holdemani, P. idalimus, P. microdorus, P. nanus, P. neoamblycephalus, P. straeleni and P. veruculatus, are unequivocally linked to the D2-D3 of 28S, ITS, 18S rRNA and COI gene sequences. Combined with scanning electron microscopy and a molecular analysis of an additional nine known and thirteen unknown species originating from diverse geographic regions, a total of 92 D2-D3 of 28S, 41 ITS, 57 $18 \mathrm{~S}$ rRNA and 111 COI new gene sequences are presented. Paratylenchus elachistus, P. holdemani and P. neoamblycephalus are recorded for the first time in Belgium and P. idalimus for the first time in Europe. Paratylenchus is an excellent example of an incredibly diverse yet morphologically minimalistic plantparasitic genus, and this study provides an integrated analysis of all available data, including coalescence-based molecular species delimitation, resulting in an updated Paratylenchus phylogeny and the corrective reassignment of 18 D2-D3 of 28S, 3 ITS, 3 18S rRNA and 25 COI gene sequences that were previously unidentified or incorrectly classified.

Keywords: D2-D3 of 28S; ITS; 18S; COI; morphology; morphometrics; Paratylenchus; plant-parasitic nematodes; phylogeny; taxonomy

\section{Introduction}

The plant-parasitic nematode (PPN) genus Paratylenchus Micoletzky, 1922, commonly known as pin nematodes, are obligate ectoparasites of a wide variety of plants, including herbs, shrubs and trees, that are distributed worldwide and cause various symptoms in their host plants [1-5]. This genus was reviewed by Tarjan [6], who provided the first key to the species. In subsequent years, several attempts were made to split the genus and group its representatives into new genera. The genus Gracilacus Raski, 1962, was proposed for members of the Paratylenchus species with stylet lengths longer than $48 \mu \mathrm{m}$ [7]. The validity of Gracilacus was supported by Thorne and Malek [8], Raski and Luc [9], Maggenti et al. [10], Raski [11], Esser [12], Andrássy [13] and Yu et al. [14], while Siddiqi [15] treated it as a subgenus of Paratylenchus. Gracilacus was synonymised with Paratylenchus by Brzeski [16], and it was recognized in further works of Siddiqi and Goodey [17], Geraert [18], Brzeski [19], Nguyen et al. [20], Decraemer and Hunt [21], Van den Berg et al. [22], Ghaderi et al. [23], 
Hesar et al. [24] and Maria et al. [25]. The genus Paratylenchoides Raski, 1973 was assigned to Paratylenchus species with stronger cephalic sclerotisations, dorso-ventrally narrower heads and small narrow rounded protrusions on the anterior surface of conoid lip region [26]. Siddiqi [15] subsequently lowered Paratylenchoides to a sub-generic level for Paratylenchus, while Raski and Luc [9] synonymised the two genera owing to the apparent lack of morphological differences between them and Siddiqi [2] accepted this. It was proposed that another genus, Gracilpaurus Ganguly and Khan, 1990, included four species displaying long stylets and tubercles on the surface of the cuticle [27]. However, Brzeski [19] did not consider cuticular ornamentation as a generic characteristic, a decision that led to the synonymising of Gracilpaurus. The monotypic genus Cacopaurus Thorne, 1943 was also proposed and distinguished from Paratylenchus by the obese female body, tubercles on annuli of the female cuticle and sessile parasitism [28]. Although Goodey [29] synonymised Cacopaurus with Paratylenchus due to the lack of consistent differential traits-apart from the female of the former sometimes being sessile and slightly swollen-Cacopaurus has been, nevertheless, accepted by Raski [7], Raski and Luc [9], Ebsary [30], Raski [11], Brzeski [19], Siddiqi [2], Andrassy [13] and Ghaderi et al. [23,31].

Nematodes of the genus Paratylenchus in a broad sense or sensu lato are characterised by: small size $(<0.7 \mathrm{~mm})$; females being vermiform to obese; $\mathrm{C}, \mathrm{J}$ or 6 shapes when heat relaxed; two to four lateral lines; cuticle with or without ornamentations; often continuous cephalic regions of rounded to conoid, truncate or trapezoid shapes; protruding or non-protruding submedian lobes; stylet lengths ranging between 10 and $120 \mu \mathrm{m}$; welldeveloped valves of median bulb, slender isthmuses and rounded to pyriform end bulbs in female pharynges; secretory-excretory pores are often at the level between median bulb and end bulb; spermathecae with or without sperm cells; commonly swollen prevulval region; vulvae with or without lateral flaps; presence or absence of a short post-vulval uterine sac; tails ranging from conoid to hemispherical with variable tail termini. The diagnostic traits of juveniles and males are less frequently used for identification, except for looking for the presence of a stylet and looking at the length of the spicules of males.

Recently, Ghaderi et al. [23] recognized 117 species of Paratylenchus sensu lato (excluding Cacopaurus), six species of inquirendae and four of nomina nuda. The nominal species were pragmatically divided into eleven groups based on stylet length, number of lateral lines and absence vs. presence of vulval flaps in females. Since then, seven more species of Paratylenchus have been described and linked to DNA sequences [14,25,32-36]. Molecular work on this nematode group is gaining momentum and provides an attractive solution to difficulties encountered in species identification, as well as phylogenetic relationships among species. Subbotin et al. [37], Chen et al. [38,39] and van Megen et al. [40] started to molecularly characterise some Paratylenchus spp. using the D2-D3 of $28 \mathrm{~S}$ rRNA, ITS rRNA and $18 \mathrm{~S}$ rRNA gene sequences, respectively. Lopez et al. [41] used ITS rRNA gene to examine phylogenetic relationships among four nematode genera; two of the included genera were Paratylenchus and Gracilacus. Van den Berg et al. [22] conducted the first comprehensive phylogenetic study including several Paratylenchus spp. by using $5828 \mathrm{~S}$ rRNA and 40 ITS rRNA gene sequences. Several other studies provided additional molecular characterisations, phylogenetic analyses and descriptions of new Paratylenchus species [14,25,32-36,42-50]. In a study by Hesar et al. [24], 28S rRNA and ITS rRNA gene sequences of several Paratylenchus spp. as well as Cacopaurus pestis Thorne, 1943, were updated. In addition to providing the first molecular characterisation of C. pestis, their phylogenetic analyses based on the two partial gene sequences did not support the monophyly of the genera Cacopaurus, Gracilacus and Gracilpaurus that were all found embedded within the clade of Paratylenchus.

Despite these recent efforts to integrate and include molecular information in species descriptions and species delineations of Paratylenchus, several taxonomic challenges still remain. This is often the case in the field of nematology in general, but the genus Paratylenchus is a perfect case in point. Most of the traditionally described species are not yet linked to molecular data, numerous sequences that are currently available are not linked to 
established species and/or morphological information, sequences are often misplaced and the existence of cryptic species within the genus is common.

Species boundaries in Paratylenchus are sometimes difficult to delimit based solely on morphology because of the limited diagnostic features and morphological plasticity. As of December 2020, only 40 Paratylenchus sensu lato species have been linked to molecular data in the GenBank and this database also includes several putative, new, unidentified and incorrectly classified sequences. These misidentified sequences may result in a cascade of erroneous interpretations, including incorrect morphological identification [51] and flawed interpretations of species identity based on relationships in phylogenetic trees. Cryptic species are also likely to represent a component of Paratylenchus diversity [22]. It is important to note that correct differentiation of species belonging to agricultural nematode pests from its sibling species has gained importance for a number of reasons, including food security, quarantine regulations and nonchemical pest management strategies [52].

The aims of this study are to: (1) provide and update molecular barcodes of several known and unknown Paratylenchus species using four partial sequences-D2-D3 of 28S, ITS and 18S rRNA gene and COI gene of mtDNA; (2) link these molecular data to comprehensive morphological information, including light microscopy (LM) and scanning electron microscopy (SEM) images and morphometrics; (3) reconstruct an updated Paratylenchus phylogeny; (4) provide a molecular species delimitation for all four markers; (5) reassign unidentified and/or incorrectly classified GenBank sequences to the appropriate species.

\section{Results}

\subsection{Species Identification, Characterisation and Delimitation}

Ten identified and four unidentified Paratylenchus species, recovered from soil samples collected in Belgium, were morphologically and molecularly characterised. The identified species were Paratylenchus aculentus Brown, 1959, Paratylenchus elachistus Steiner, 1949, Paratylenchus goodeyi Oostenbrink, 1953, Paratylenchus holdemani Raski, 1975, Paratylenchus idalimus (Raski, 1962) Siddiqi and Goodey, 1964, Paratylenchus microdorus Andrássy, 1959, Paratylenchus nanus Cobb, 1923, Paratylenchus neoamblycephalus Geraert, 1965, Paratylenchus straeleni (De Coninck, 1931) Oostenbrink, 1960 and Paratylenchus veruculatus Wu, 1962. The unidentified Paratylenchus spp. were Paratylenchus sp.2, Paratylenchus sp.BE11, Paratylenchus sp.D, and Paratylenchus sp.F. Paratylenchus elachistus, P. holdemani, P. idalimus and P. neoamblycephalus were reported for the first time in Belgium and P. idalimus was recorded for the first time in Europe. Additional sequences of Paratylenchus aquaticus Merny, 1966, Paratylenchus dianthus Jenkins and Tylor, 1956, Paratylenchus hamatus Thorne and Allen, 1950, Paratylenchus leptos Raski 1975, P. nanus, Paratylenchus projectus Jenkins 1956, Paratylenchus shenzhenensis Wang, Xie, Li, Xu, Yu and Wang, 2013, P. straeleni and Paratylenchus tenuicaudatus $\mathrm{Wu}, 1961$ and thirteen unidentified Paratylenchus species that originated from diverse geographic regions are also provided (Table 1). In total, 68 D2-D3 of 28S, 38 ITS, 57 18S rRNA and 84 COI gene sequences were linked to morphological data of the abovementioned ten known and four unknown species collected from Belgium, and 24 D2-D3 of 28S, 3 ITS rRNA and 27 COI gene sequences were added to the other nine known and thirteen unidentified species. 
Table 1. List of Paratylenchus populations included in this study. Accession numbers of three ribosomal RNA genes (D2-D3 of 28S, ITS and 18S) and a mitochondrial gene (COI) fragments are provided for 18 identified and 14 unidentified Paratylenchus species. Accession numbers in italics are ones generated in this study.

\begin{tabular}{|c|c|c|c|c|c|c|c|c|}
\hline \multirow[b]{2}{*}{ Species } & \multirow[b]{2}{*}{ Locality } & \multirow{2}{*}{$\begin{array}{l}\text { Associated } \\
\text { Plant Host }\end{array}$} & \multirow[b]{2}{*}{$\begin{array}{l}\text { Sample } \\
\text { Code }\end{array}$} & \multicolumn{4}{|c|}{ GenBank Accession Numbers } & \multirow[b]{2}{*}{ Source } \\
\hline & & & & $\begin{array}{c}28 S \\
\text { rRNA }\end{array}$ & $\begin{array}{l}\text { ITS } \\
\text { rRNA }\end{array}$ & $\begin{array}{c}18 S \\
\text { rRNA }\end{array}$ & $\begin{array}{l}\text { COI of } \\
\text { mtDNA }\end{array}$ & \\
\hline P. aculentus & $\begin{array}{l}\text { Belgium, } \\
\text { Ghent, } \\
\text { Citadel Park; } \\
51^{\circ} 02^{\prime} 05^{\prime \prime} \mathrm{N} ; \\
3^{\circ} 43^{\prime} 10^{\prime \prime} \mathrm{E}\end{array}$ & $\begin{array}{c}\text { Grasses } \\
\text { under } \\
\text { a tree }\end{array}$ & BE9 & $\begin{array}{c}M W 413626- \\
M W 41328\end{array}$ & $\begin{array}{l}M W 413588- \\
M W 413589\end{array}$ & $\begin{array}{c}M W 413692- \\
M W 413693\end{array}$ & $\begin{array}{c}M W 421639- \\
M W 421642\end{array}$ & $\begin{array}{c}\text { C.M. } \\
\text { Etongwe }\end{array}$ \\
\hline $\begin{array}{c}\text { P. aquaticus } \\
\text { type A }\end{array}$ & $\begin{array}{l}\text { USA, Florida, } \\
\text { Princeton }\end{array}$ & Aechmea sp. & CD3375 & MW413557 & - & - & - & $\begin{array}{c}\text { S.A. } \\
\text { Subbotin }\end{array}$ \\
\hline $\begin{array}{c}\text { P. aquaticus } \\
\text { type A }\end{array}$ & $\begin{array}{c}\text { USA, } \\
\text { Hawaii, } \\
\text { Waimanalo }\end{array}$ & $\begin{array}{c}\text { Bromeliad } \\
\text { (Neoregelia } \\
\text { sp.) }\end{array}$ & CD619 & $\begin{array}{l}\text { KF242239, } \\
\text { KF242240 }\end{array}$ & $\begin{array}{l}\text { KF242277, } \\
\text { KF242278 }\end{array}$ & - & MW411845 & $\begin{array}{c}\text { S.A. } \\
\text { Subbotin, } \\
\text { Van den Berg } \\
\text { et al. [22] }\end{array}$ \\
\hline $\begin{array}{c}\text { P. aquaticus } \\
\text { type B }\end{array}$ & $\begin{array}{l}\text { USA, Kansas, } \\
\text { Manhattan, } \\
\text { Washinton- } \\
\text { Marlatt } \\
\text { park }\end{array}$ & Grasses & CD868 & KF242241 & - & - & MW411838 & $\begin{array}{c}\text { S.A. } \\
\text { Subbotin, } \\
\text { Van den Berg } \\
\text { et al. [22] }\end{array}$ \\
\hline P. dianthus & $\begin{array}{c}\text { South Africa, } \\
\text { Gauteng, } \\
\text { Tarlton }\end{array}$ & Chrysanthemum & CD552 & $\begin{array}{l}\text { KF242226- } \\
\text { KF242229 }\end{array}$ & $\begin{array}{l}\text { KF242271, } \\
\text { KF242272 }\end{array}$ & - & MW411837 & $\begin{array}{l}\text { Van den } \\
\text { Berg, Van } \\
\text { den Berg } \\
\text { et al. [22] }\end{array}$ \\
\hline P. enigmaticus & $\begin{array}{c}\text { Belgium, } \\
\text { Ghent, } \\
\text { Ghent } \\
\text { University } \\
\text { Botanical } \\
\text { Garden; } \\
51^{\circ} 2^{\prime} 7.53^{\prime \prime} \mathrm{N} ; \\
3^{\circ} 43^{\prime} 20.07^{\prime \prime} \mathrm{E}\end{array}$ & Leek & BE2 & - & $\begin{array}{l}M W 413621- \\
M W 413622\end{array}$ & $\begin{array}{c}\text { MW413735, } \\
\text { MW413737- } \\
\text { MW413739 }\end{array}$ & MW421686 & $\begin{array}{c}\text { C.M. } \\
\text { Etongwe }\end{array}$ \\
\hline P. enigmaticus & $\begin{array}{c}\text { Belgium, } \\
\text { Ghent, } \\
\text { Ghent } \\
\text { University } \\
\text { Botanical } \\
\text { Garden; } \\
51^{\circ} 2^{\prime} 7.10^{\prime \prime} \mathrm{N} ; \\
3^{\circ} 43^{\prime} 19.28^{\prime \prime} \mathrm{E}\end{array}$ & $\begin{array}{c}\text { Wild } \\
\text { oregano }\end{array}$ & BE4 & - & - & $\begin{array}{c}\text { MW413732- } \\
\text { MW413734 }\end{array}$ & - & $\begin{array}{c}\text { C.M. } \\
\text { Etongwe }\end{array}$ \\
\hline P. enigmaticus & USA, Idaho & $\begin{array}{c}\text { Unknown } \\
\text { plant }\end{array}$ & CD2485 & MW413568 & MW413583 & - & MW411828 & $\begin{array}{c}\text { S.A. } \\
\text { Subbotin }\end{array}$ \\
\hline P. elachistus & $\begin{array}{l}\text { Belgium, } \\
\text { Kortrijk; } \\
50^{\circ} 47^{\prime} 58^{\prime \prime} \mathrm{N} ; \\
3^{\circ} 11^{\prime} 37^{\prime \prime} \mathrm{E}\end{array}$ & $\begin{array}{c}\text { Grasses } \\
\text { under } \\
\text { a thorny tree }\end{array}$ & BE15 & $\begin{array}{c}M W 413629- \\
M W 413630\end{array}$ & $\begin{array}{c}M W 413590- \\
M W 413593\end{array}$ & $\begin{array}{l}\text { MW413694- } \\
\text { MW413697 }\end{array}$ & $\begin{array}{c}M W 421643- \\
M W 421646\end{array}$ & $\begin{array}{c}\text { C.M. } \\
\text { Etongwe }\end{array}$ \\
\hline P. goodeyi & $\begin{array}{l}\text { Belgium, } \\
\text { Merendree; } \\
51^{\circ} 04^{\prime} 12^{\prime \prime} \mathrm{N} \text {; } \\
3^{\circ} 34^{\prime} 37^{\prime \prime} \mathrm{E}\end{array}$ & $\begin{array}{c}\text { Grasses } \\
\text { around a } \\
\text { beech tree }\end{array}$ & BE22 & $\begin{array}{c}M W 413631- \\
M W 413633\end{array}$ & MW413594 & $\begin{array}{l}\text { MW413698- } \\
\text { MW413699 }\end{array}$ & $\begin{array}{l}\text { MW421647- } \\
\text { MW421649 }\end{array}$ & $\begin{array}{c}\text { C.M. } \\
\text { Etongwe }\end{array}$ \\
\hline P. hamatus & $\begin{array}{c}\text { USA, } \\
\text { California, } \\
\text { Merced } \\
\text { County, } \\
\text { Planada }\end{array}$ & $\begin{array}{c}\text { Fig tree } \\
\text { (Ficus carica) }\end{array}$ & CD1155 & KF242212 & - & - & MW411821 & $\begin{array}{c}\text { S.A. } \\
\text { Subbotin, } \\
\text { Van den Berg } \\
\text { et al. [22] }\end{array}$ \\
\hline P. hamatus & $\begin{array}{c}\text { USA, } \\
\text { California }\end{array}$ & Trees & CD1914 & MW413564 & MW413585 & - & MW411823 & $\begin{array}{c}\text { S.A. } \\
\text { Subbotin }\end{array}$ \\
\hline
\end{tabular}


Table 1. Cont.

\begin{tabular}{|c|c|c|c|c|c|c|c|c|}
\hline \multirow[b]{2}{*}{ Species } & \multirow[b]{2}{*}{ Locality } & \multirow[b]{2}{*}{$\begin{array}{l}\text { Associated } \\
\text { Plant Host }\end{array}$} & \multirow[b]{2}{*}{$\begin{array}{l}\text { Sample } \\
\text { Code }\end{array}$} & \multicolumn{4}{|c|}{ GenBank Accession Numbers } & \multirow[b]{2}{*}{ Source } \\
\hline & & & & $\begin{array}{l}\text { 28S } \\
\text { rRNA }\end{array}$ & $\begin{array}{l}\text { ITS } \\
\text { rRNA }\end{array}$ & $\begin{array}{c}\text { 18S } \\
\text { rRNA }\end{array}$ & $\begin{array}{l}\text { COI of } \\
\text { mtDNA }\end{array}$ & \\
\hline P. hamatus & $\begin{array}{c}\text { USA, } \\
\text { California, } \\
\text { Kern county }\end{array}$ & $\begin{array}{c}\text { Grape } \\
\text { (Vitis sp.) }\end{array}$ & CD2534a, b & $\begin{array}{l}\text { MW413565, } \\
\text { MW413566 }\end{array}$ & - & - & - & A. Westphal \\
\hline P. hamatus & $\begin{array}{c}\text { USA, } \\
\text { California, } \\
\text { Kern county, } \\
\text { Delano }\end{array}$ & $\begin{array}{l}\text { Grape, } \\
\text { Cherry }\end{array}$ & CD3372 & MW413558 & - & - & - & $\begin{array}{c}\text { S.A. } \\
\text { Subbotin }\end{array}$ \\
\hline P. hamatus & $\begin{array}{c}\text { USA, } \\
\text { California, } \\
\text { Kern county, } \\
\text { Maricopa }\end{array}$ & $\begin{array}{c}\text { Apricot } \\
\text { (Prunus sp.) }\end{array}$ & CD454 & $\begin{array}{l}\text { KF242206, } \\
\text { KF242216, } \\
\text { KF242217 }\end{array}$ & $\begin{array}{l}\text { KF242247, } \\
\text { KF242256 }\end{array}$ & - & MW411822 & $\begin{array}{c}\text { S.A. } \\
\text { Subbotin, } \\
\text { Van den Berg } \\
\text { et al. [22] }\end{array}$ \\
\hline P. holdemani & $\begin{array}{c}\text { Belgium, } \\
\text { Gouvy, } \\
\text { Rogery; } \\
50^{\circ} 14^{\prime} 39.8^{\prime \prime} \mathrm{N} ; \\
5^{\circ} 57^{\prime} 21.9^{\prime \prime} \mathrm{E}\end{array}$ & $\begin{array}{l}\text { Grasses } \\
\text { under tree } \\
\text { Fraxinus sp. }\end{array}$ & AR3 & $\begin{array}{l}\text { MW413636- } \\
M W 413638 \\
M W 413640 \\
M W 413642\end{array}$ & - & MW413701 & $\begin{array}{l}\text { MW421650- } \\
\text { MW421652 }\end{array}$ & P.R. Singh \\
\hline P. holdemani & $\begin{array}{c}\text { Belgium, } \\
\text { Ghent, } \\
\text { Blaarmeersen; } \\
51^{\circ} 02^{\prime} 18.9^{\prime \prime} \mathrm{N} ; \\
3^{\circ} 41^{\prime} 17.2^{\prime \prime} \mathrm{E}\end{array}$ & $\begin{array}{l}\text { Grasses } \\
\text { under a } \\
\text { thorny tree } \\
\text { next to a } \\
\text { stream }\end{array}$ & BE19 & $\begin{array}{l}\text { MW413634- } \\
\text { MW413635 }\end{array}$ & MW413595 & MW413700 & MW421658 & $\begin{array}{l}\text { C.M. } \\
\text { Etongwe }\end{array}$ \\
\hline P. holdemani & $\begin{array}{l}\text { Belgium, } \\
\text { Ghent, } \\
\text { Blaarmeersen; } \\
51^{\circ} 02^{\prime} 14^{\prime \prime} \mathrm{N} ; \\
3^{\circ} 41^{\prime} 23^{\prime \prime} \mathrm{E}\end{array}$ & $\begin{array}{l}\text { Grasses } \\
\text { under } \\
\text { a tree }\end{array}$ & BE20 & $\begin{array}{l}\text { MW413639, } \\
\text { MW413641 }\end{array}$ & MW413596 & MW413702 & $\begin{array}{l}\text { MW421653- } \\
\text { MW421657 }\end{array}$ & $\begin{array}{l}\text { C.M. } \\
\text { Etongwe }\end{array}$ \\
\hline P. idalimus & $\begin{array}{c}\text { Belgium, } \\
\text { Ghent, } \\
\text { Blaarmeersen; } \\
51^{\circ} 02^{\prime} 18.9^{\prime \prime} \mathrm{N} ; \\
3^{\circ} 41^{\prime} 17.2^{\prime \prime} \mathrm{E}\end{array}$ & $\begin{array}{l}\text { Grasses } \\
\text { under a } \\
\text { thorny tree } \\
\text { next to a } \\
\text { stream }\end{array}$ & BE19 & MW413644 & - & - & - & $\begin{array}{l}\text { C.M. } \\
\text { Etongwe }\end{array}$ \\
\hline P. idalimus & $\begin{array}{l}\text { Belgium, } \\
\text { Ghent, } \\
\text { Blaarmeersen; } \\
51^{\circ} 02^{\prime} 14^{\prime \prime} \mathrm{N} ; \\
3^{\circ} 41^{\prime} 23^{\prime \prime} \mathrm{E}\end{array}$ & $\begin{array}{l}\text { Grassesundera } \\
\text { tree }\end{array}$ & BE20 & MW413643 & - & $\begin{array}{l}\text { MW413703- } \\
\text { MW413704 }\end{array}$ & - & $\begin{array}{l}\text { C.M. } \\
\text { Etongwe }\end{array}$ \\
\hline P. idalimus & $\begin{array}{c}\text { USA, } \\
\text { California, } \\
\text { Napa county, } \\
\text { Napa }\end{array}$ & $\begin{array}{c}\text { Grape } \\
\text { (Vitis sp.) }\end{array}$ & CD106 & $\begin{array}{l}\text { KF242237, } \\
\text { KF242238 }\end{array}$ & $\begin{array}{l}\text { KF242275, } \\
\text { KF242276 }\end{array}$ & - & MW411839 & $\begin{array}{c}\text { S.A. } \\
\text { Subbotin, } \\
\text { Van den Berg } \\
\text { et al. [22] }\end{array}$ \\
\hline P. leptos & $\begin{array}{c}\text { Ethiopia, } \\
\text { Jimma Zone, } \\
\text { Gera district }\end{array}$ & Coffee & Ge16c & $\begin{array}{c}\text { MW413645- } \\
\text { MW413653 }\end{array}$ & - & - & $\begin{array}{l}\text { MW421659- } \\
\text { MW421665 }\end{array}$ & A.W. Aseffa \\
\hline P. microdorus & $\begin{array}{c}\text { Belgium, } \\
\text { Zwijnaarde; } \\
51^{\circ} 00^{\prime} 19^{\prime \prime} \mathrm{N} ; \\
3^{\circ} 42^{\prime} 11^{\prime \prime} \mathrm{E}\end{array}$ & Grasses & BE11 & - & MW413597 & - & - & $\begin{array}{c}\text { C.M. } \\
\text { Etongwe }\end{array}$ \\
\hline P. microdorus & $\begin{array}{l}\text { Belgium, } \\
\text { Ghent, } \\
\text { Citadel Park; } \\
51^{\circ} 02^{\prime} 05^{\prime \prime} \mathrm{N} ; \\
3^{\circ} 43^{\prime} 10^{\prime \prime} \mathrm{E}\end{array}$ & $\begin{array}{l}\text { Grasses } \\
\text { under } \\
\text { a tree }\end{array}$ & BE9 & $\begin{array}{c}\text { MW413654- } \\
\text { MW413655 }\end{array}$ & $\begin{array}{l}\text { MW413598- } \\
\text { MW413600 }\end{array}$ & $\begin{array}{l}\text { MW413705- } \\
\text { MW413706 }\end{array}$ & $\begin{array}{l}\text { MW421666- } \\
\text { MW421667 }\end{array}$ & $\begin{array}{l}\text { C.M. } \\
\text { Etongwe }\end{array}$ \\
\hline
\end{tabular}


Table 1. Cont.

\begin{tabular}{|c|c|c|c|c|c|c|c|c|}
\hline \multirow[b]{2}{*}{ Species } & \multirow[b]{2}{*}{ Locality } & \multirow{2}{*}{$\begin{array}{l}\text { Associated } \\
\text { Plant Host }\end{array}$} & \multirow{2}{*}{$\begin{array}{c}\text { Sample } \\
\text { Code }\end{array}$} & \multicolumn{4}{|c|}{ GenBank Accession Numbers } & \multirow[b]{2}{*}{ Source } \\
\hline & & & & $\begin{array}{c}28 S \\
\text { rRNA }\end{array}$ & $\begin{array}{l}\text { ITS } \\
\text { rRNA }\end{array}$ & $\begin{array}{c}\text { 18S } \\
\text { rRNA }\end{array}$ & $\begin{array}{l}\text { COI of } \\
\text { mtDNA }\end{array}$ & \\
\hline P. nanus & $\begin{array}{c}\text { USA, } \\
\text { California, } \\
\text { Humboldt } \\
\text { county, } \\
\text { Trinidad, } \\
\text { sample 5B; } \\
41^{\circ} 02^{\prime} 40.6^{\prime \prime} \mathrm{N} ; \\
124^{\circ} 07^{\prime} 18.1^{\prime \prime} \mathrm{W}\end{array}$ & $\begin{array}{l}\text { Unknown } \\
\text { plant }\end{array}$ & CD3141 & MW413576 & - & - & - & $\begin{array}{c}\text { S.A. } \\
\text { Subbotin }\end{array}$ \\
\hline P. nanus & $\begin{array}{c}\text { USA, } \\
\text { Washington, } \\
\text { Mason } \\
\text { County, } \\
\text { Skokomish, } \\
\text { sample 32B; } \\
47^{\circ} 18^{\prime} 07.0^{\prime \prime} \mathrm{N} ; \\
123^{\circ} 10^{\prime} 95.6^{\prime \prime} \mathrm{W}\end{array}$ & $\begin{array}{l}\text { Unknown } \\
\text { plant }\end{array}$ & CD3217 & MW413575 & - & - & - & $\begin{array}{c}\text { S.A. } \\
\text { Subbotin }\end{array}$ \\
\hline P. nanus & $\begin{array}{l}\text { USA, } \\
\text { California, } \\
\text { Riverside }\end{array}$ & Grasses & CD728 & $\begin{array}{l}\text { KF242194, } \\
\text { KF242197 }\end{array}$ & $\begin{array}{l}\text { KF242267, } \\
\text { KF242268 }\end{array}$ & - & MW411835 & $\begin{array}{c}\text { S.A. } \\
\text { Subbotin, } \\
\text { Van den Berg } \\
\text { et al. [22] }\end{array}$ \\
\hline P. nanus & $\begin{array}{c}\text { USA, } \\
\text { California, } \\
\text { Marin } \\
\text { county }\end{array}$ & Festuca sp. & CD850 & $\begin{array}{l}\text { KF242192, } \\
\text { KF242193 }\end{array}$ & - & - & MW411834 & $\begin{array}{c}\text { S.A. } \\
\text { Subbotin, } \\
\text { Van den Berg } \\
\text { et al. [22] }\end{array}$ \\
\hline P. nanus & $\begin{array}{l}\text { USA, } \\
\text { California, } \\
\text { Marin } \\
\text { county }\end{array}$ & Grasses & CD860 & $\begin{array}{l}\text { KF242191, } \\
\text { KF242195 }\end{array}$ & - & - & MW411836 & $\begin{array}{c}\text { S.A. } \\
\text { Subbotin, } \\
\text { Van den Berg } \\
\text { et al. [22] }\end{array}$ \\
\hline P. nanus & $\begin{array}{c}\text { Belgium, } \\
\text { Gouvy, } \\
\text { Rogery; } 50^{\circ} 14^{\prime} 39.8 \\
5^{\circ} 57^{\prime} 21.9^{\prime \prime} \mathrm{E}\end{array}$ & $\begin{array}{c}\text { Grasses } \\
\text { 8under tree } \\
\text { 8raxinus sp. }\end{array}$ & AR3 & - & - & - & MW421673 & P.R. Singh \\
\hline P. nanus & $\begin{array}{c}\text { Belgium, } \\
\text { Zwijnaarde; } \\
51^{\circ} 00^{\prime} 19^{\prime \prime} \mathrm{N} ; \\
3^{\circ} 42^{\prime} 11^{\prime \prime} \mathrm{E}\end{array}$ & $\begin{array}{l}\text { Grasses } \\
\text { under } \\
\text { a tree }\end{array}$ & BE11 & $\begin{array}{l}M W 413658- \\
M W 413659\end{array}$ & $\begin{array}{l}\text { MW413601- } \\
\text { MW413603 }\end{array}$ & $\begin{array}{c}\text { MW413707, } \\
\text { MW413711- } \\
\text { MW413712 }\end{array}$ & $\begin{array}{l}\text { MW421668- } \\
\text { MW421671, } \\
\text { MW421674 }\end{array}$ & $\begin{array}{c}\text { C.M. } \\
\text { Etongwe }\end{array}$ \\
\hline P. nanus & $\begin{array}{l}\text { Belgium, } \\
\text { Ghent, } \\
\text { Blaarmeersen; } \\
51^{\circ} 07^{\prime} 14^{\prime \prime} \mathrm{N} ; \\
2^{\circ} 39^{\prime} 29^{\prime \prime} \mathrm{E}\end{array}$ & $\begin{array}{l}\text { Grasses } \\
\text { under } \\
\text { a tree }\end{array}$ & BE18 & MW413657 & MW413604 & MW413708 & MW421672 & $\begin{array}{c}\text { C.M. } \\
\text { Etongwe }\end{array}$ \\
\hline $\begin{array}{l}\text { P. neoambly- } \\
\text { cephalus }\end{array}$ & $\begin{array}{l}\text { Belgium, } \\
\text { Ghent, } \\
\text { Citadel Park; } \\
51^{\circ} 02^{\prime} 09^{\prime \prime} \mathrm{N} ; \\
3^{\circ} 43^{\prime} 06^{\prime \prime} \mathrm{E}\end{array}$ & $\begin{array}{l}\text { Cypress } \\
\text { tree }\end{array}$ & BE10 & $\begin{array}{l}\text { MW413660- } \\
\text { MW413663 }\end{array}$ & $\begin{array}{l}\text { MW413606- } \\
\text { MW413610 }\end{array}$ & $\begin{array}{l}\text { MW413713- } \\
\text { MW413718 }\end{array}$ & $\begin{array}{l}\text { MW421675- } \\
\text { MW421682 }\end{array}$ & $\begin{array}{c}\text { C.M. } \\
\text { Etongwe }\end{array}$ \\
\hline $\begin{array}{l}\text { P. neoambly- } \\
\text { cephalus }\end{array}$ & $\begin{array}{c}\text { USA, } \\
\text { California, } \\
\text { Madera } \\
\text { county, } \\
\text { Madera }\end{array}$ & Grasses & CD1223 & KF242190 & - & - & MW411843 & $\begin{array}{c}\text { S.A. } \\
\text { Subbotin, } \\
\text { Van den Berg } \\
\text { et al. [22] }\end{array}$ \\
\hline
\end{tabular}


Table 1. Cont.

\begin{tabular}{|c|c|c|c|c|c|c|c|c|}
\hline \multirow[b]{2}{*}{ Species } & \multirow[b]{2}{*}{ Locality } & \multirow[b]{2}{*}{$\begin{array}{l}\text { Associated } \\
\text { Plant Host }\end{array}$} & \multirow[b]{2}{*}{$\begin{array}{l}\text { Sample } \\
\text { Code }\end{array}$} & \multicolumn{4}{|c|}{ GenBank Accession Numbers } & \multirow[b]{2}{*}{ Source } \\
\hline & & & & $\begin{array}{l}\text { 28S } \\
\text { rRNA }\end{array}$ & $\begin{array}{l}\text { ITS } \\
\text { rRNA }\end{array}$ & $\begin{array}{c}\text { 18S } \\
\text { rRNA }\end{array}$ & $\begin{array}{l}\text { COI of } \\
\text { mtDNA }\end{array}$ & \\
\hline P. projectus & $\begin{array}{c}\text { Belgium, } \\
\text { Ghent, } \\
\text { Ghent } \\
\text { University } \\
\text { Botanical } \\
\text { Garden; } \\
51^{\circ} 2^{\prime} 7.53^{\prime \prime} \mathrm{N} ; \\
3^{\circ} 43^{\prime} 20.07^{\prime \prime} \mathrm{E}\end{array}$ & Leek & BE2 & MW413656 & MW413605 & $\begin{array}{l}\text { MW413709- } \\
\text { MW413710 }\end{array}$ & - & $\begin{array}{c}\text { C.M. } \\
\text { Etongwe }\end{array}$ \\
\hline P. projectus & $\begin{array}{c}\text { USA, } \\
\text { California, } \\
\text { Butte county, } \\
\text { Gridley }\end{array}$ & $\begin{array}{c}\text { Walnut } \\
\text { (Juglans sp.) }\end{array}$ & CD137 & KF242199 & $\begin{array}{l}\text { KF242265, } \\
\text { KF242266 }\end{array}$ & - & MW411840 & $\begin{array}{c}\text { S.A. } \\
\text { Subbotin, } \\
\text { Van den Berg } \\
\text { et al. [22] }\end{array}$ \\
\hline P. projectus & $\begin{array}{c}\text { South Africa, } \\
\text { Western } \\
\text { Cape, } \\
\text { George }\end{array}$ & Bent grass & CD587 & $\begin{array}{l}\text { KF242198, } \\
\text { KF242200 }\end{array}$ & $\begin{array}{l}\text { KF242263, } \\
\text { KF242264 }\end{array}$ & - & MW411842 & $\begin{array}{c}\text { Van den } \\
\text { Berg, Van } \\
\text { den Berg et } \\
\text { al. [22] }\end{array}$ \\
\hline $\begin{array}{c}P . \\
\text { shenzhenensis }\end{array}$ & $\begin{array}{l}\text { USA, Florida, } \\
\text { Apopka }\end{array}$ & $\begin{array}{l}\text { Unknown } \\
\text { plant }\end{array}$ & CD2728 & MW413579 & - & - & - & $\begin{array}{c}\text { S.A. } \\
\text { Subbotin }\end{array}$ \\
\hline P. straeleni & $\begin{array}{c}\text { Belgium, } \\
\text { Zwijnaarde; } \\
51^{\circ} 00^{\prime} 19^{\prime \prime} \mathrm{N} ; \\
3^{\circ} 42^{\prime} 11^{\prime \prime} \mathrm{E}\end{array}$ & $\begin{array}{c}\text { Grasses } \\
\text { under } \\
\text { a tree }\end{array}$ & BE11 & MW413686 & MW413623 & - & $\begin{array}{l}\text { MW421713- } \\
\text { MW421715 }\end{array}$ & $\begin{array}{c}\text { C.M. } \\
\text { Etongwe }\end{array}$ \\
\hline P. straeleni & $\begin{array}{c}\text { Belgium, } \\
\text { Kortrijk; } \\
50^{\circ} 47^{\prime} 58^{\prime \prime} \mathrm{N} ; \\
3^{\circ} 11^{\prime} 37^{\prime \prime} \mathrm{E}\end{array}$ & $\begin{array}{c}\text { Grasses } \\
\text { under } \\
\text { a thorny tree }\end{array}$ & BE15 & MW413685 & $\begin{array}{l}\text { MW413624- } \\
\text { MW413625 }\end{array}$ & $\begin{array}{l}\text { MW413743- } \\
\text { MW413746 }\end{array}$ & $\begin{array}{l}\text { MW421708- } \\
\text { MW421712 }\end{array}$ & $\begin{array}{c}\text { C.M. } \\
\text { Etongwe }\end{array}$ \\
\hline P. straeleni & $\begin{array}{c}\text { Belgium, } \\
\text { Ghent, } \\
\text { Blaarmeersen; } \\
51^{\circ} 02^{\prime} 18.9^{\prime \prime} \mathrm{N} ; \\
3^{\circ} 41^{\prime} 17.2^{\prime \prime} \mathrm{E}\end{array}$ & $\begin{array}{l}\text { Grasses } \\
\text { under } \\
\text { a thorny tree } \\
\text { next to a } \\
\text { stream }\end{array}$ & BE19 & - & - & - & MW421716 & $\begin{array}{c}\text { C.M. } \\
\text { Etongwe }\end{array}$ \\
\hline P. straeleni & $\begin{array}{c}\text { USA, } \\
\text { North } \\
\text { Carolina }\end{array}$ & $\begin{array}{l}\text { Unknown } \\
\text { plant }\end{array}$ & CD1433 & MW413577 & - & - & - & W. Ye \\
\hline P. straeleni & $\begin{array}{c}\text { USA, } \\
\text { California, } \\
\text { Monterey } \\
\text { county }\end{array}$ & Oak & CD1775 & MW413578 & - & - & MW411831 & $\begin{array}{c}\text { S.A. } \\
\text { Subbotin }\end{array}$ \\
\hline P. straeleni & $\begin{array}{c}\text { USA, } \\
\text { California, } \\
\text { Napa county }\end{array}$ & Tree & CD899 & KF242236 & - & - & MW411832 & $\begin{array}{c}\text { S.A. } \\
\text { Subbotin, } \\
\text { Van den Berg } \\
\text { et al. [22] }\end{array}$ \\
\hline $\begin{array}{c}P . \\
\text { tenuicaudatus }\end{array}$ & $\begin{array}{l}\text { USA, } \\
\text { California, } \\
\text { Glenn } \\
\text { county, } \\
\text { Orland }\end{array}$ & $\begin{array}{c}\text { Prune } \\
\text { (Prunus sp.) }\end{array}$ & CD57 & $\begin{array}{l}\text { KF242223, } \\
\text { KF242225 }\end{array}$ & $\begin{array}{l}\text { KF242261, } \\
\text { KF242262 }\end{array}$ & - & MW411826 & $\begin{array}{c}\text { S.A. } \\
\text { Subbotin, } \\
\text { Van den Berg } \\
\text { et al. [22] }\end{array}$ \\
\hline $\begin{array}{c}P . \\
\text { tenuicaudatus }\end{array}$ & $\begin{array}{c}\text { USA, } \\
\text { California, } \\
\text { Glenn } \\
\text { county, } \\
\text { Butte City }\end{array}$ & $\begin{array}{c}\text { Prune } \\
\text { (Prunus sp.) }\end{array}$ & CD61 & KF242224 & $\begin{array}{l}\text { KF242259, } \\
\text { KF242260 }\end{array}$ & - & MW411827 & $\begin{array}{c}\text { S.A. } \\
\text { Subbotin, } \\
\text { Van den Berg } \\
\text { et al. [22] }\end{array}$ \\
\hline
\end{tabular}


Table 1. Cont.

\begin{tabular}{|c|c|c|c|c|c|c|c|c|}
\hline \multirow[b]{2}{*}{ Species } & \multirow[b]{2}{*}{ Locality } & \multirow[b]{2}{*}{$\begin{array}{l}\text { Associated } \\
\text { Plant Host }\end{array}$} & \multirow[b]{2}{*}{$\begin{array}{l}\text { Sample } \\
\text { Code }\end{array}$} & \multicolumn{4}{|c|}{ GenBank Accession Numbers } & \multirow[b]{2}{*}{ Source } \\
\hline & & & & $\begin{array}{l}28 \mathrm{~S} \\
\text { rRNA }\end{array}$ & $\begin{array}{l}\text { ITS } \\
\text { rRNA }\end{array}$ & $\begin{array}{c}\text { 18S } \\
\text { rRNA }\end{array}$ & $\begin{array}{l}\text { COI of } \\
\text { mtDNA }\end{array}$ & \\
\hline P. veruculatus & $\begin{array}{l}\text { Belgium, } \\
\text { Ghent, } \\
\text { Blaarmeersen; } \\
51^{\circ} 02^{\prime} 14^{\prime \prime} \mathrm{N} ; \\
3^{\circ} 41^{\prime} 23^{\prime \prime} \mathrm{E}\end{array}$ & $\begin{array}{c}\text { Grasses } \\
\text { under } \\
\text { a tree }\end{array}$ & BE20 & $\begin{array}{l}\text { MW413687- } \\
\text { MW413691 }\end{array}$ & - & $\begin{array}{l}\text { MW413747- } \\
\text { MW413748 }\end{array}$ & $\begin{array}{l}\text { MW421717- } \\
\text { MW421722 }\end{array}$ & $\begin{array}{l}\text { C.M. } \\
\text { Etongwe }\end{array}$ \\
\hline $\begin{array}{l}\text { Paratylenchus } \\
\text { sp.2 }\end{array}$ & $\begin{array}{c}\text { Belgium, } \\
\text { Kortrijk; } \\
50^{\circ} 47^{\prime} 58^{\prime \prime} \mathrm{N} ; \\
3^{\circ} 11^{\prime} 37^{\prime \prime} \mathrm{E}\end{array}$ & $\begin{array}{l}\text { Grasses } \\
\text { under a } \\
\text { thorny tree }\end{array}$ & BE15 & $\begin{array}{l}\text { MW413670- } \\
\text { MW413671 }\end{array}$ & $\begin{array}{l}\text { MW413615- } \\
\text { MW413616 }\end{array}$ & $\begin{array}{l}\text { MW413724- } \\
\text { MW413725 }\end{array}$ & $\begin{array}{l}\text { MW421683- } \\
\text { MW421684 }\end{array}$ & $\begin{array}{c}\text { C.M. } \\
\text { Etongwe }\end{array}$ \\
\hline $\begin{array}{l}\text { Paratylenchus } \\
\text { sp. } 2\end{array}$ & $\begin{array}{l}\text { Belgium, } \\
\text { Ghent, } \\
\text { Blaarmeersen; } \\
51^{\circ} 02^{\prime} 14^{\prime \prime} \mathrm{N} ; \\
3^{\circ} 41^{\prime} 23^{\prime \prime} \mathrm{E}\end{array}$ & $\begin{array}{l}\text { Grasses } \\
\text { under } \\
\text { a tree }\end{array}$ & BE20 & - & - & MW413726 & MW421685 & $\begin{array}{c}\text { C.M. } \\
\text { Etongwe }\end{array}$ \\
\hline $\begin{array}{l}\text { Paratylenchus } \\
\text { sp.2 }\end{array}$ & Kyrgyzstan & $\begin{array}{l}\text { Trees and } \\
\text { grasses }\end{array}$ & CD2139 & MW413567 & - & - & - & $\begin{array}{c}\text { S.A. } \\
\text { Subbotin }\end{array}$ \\
\hline $\begin{array}{l}\text { Paratylenchus } \\
\text { sp.2 }\end{array}$ & $\begin{array}{c}\text { USA, } \\
\text { California, } \\
\text { Yolo county, } \\
\text { Davis }\end{array}$ & $\begin{array}{c}\text { Grasses } \\
\text { under } \\
\text { a willow tree }\end{array}$ & CD604 & $\begin{array}{l}\text { KF242220- } \\
\text { KF242222 }\end{array}$ & KF242243 & - & MW411825 & $\begin{array}{c}\text { S.A. } \\
\text { Subbotin, } \\
\text { Van den Berg } \\
\text { et al. [22] }\end{array}$ \\
\hline $\begin{array}{l}\text { Paratylenchus } \\
\text { sp.3 }\end{array}$ & $\begin{array}{l}\text { USA, } \\
\text { California, } \\
\text { Santa } \\
\text { Barbara } \\
\text { county, } \\
\text { Goleta }\end{array}$ & $\begin{array}{l}\text { Lemon } \\
\text { (Citrus sp.) }\end{array}$ & CD232 & $\begin{array}{l}\text { KF242231, } \\
\text { KF242232 }\end{array}$ & $\begin{array}{l}\text { KF242273, } \\
\text { KF242274 }\end{array}$ & - & MW411819 & $\begin{array}{c}\text { S.A. } \\
\text { Subbotin, } \\
\text { Van den Berg } \\
\text { et al. [22] }\end{array}$ \\
\hline $\begin{array}{l}\text { Paratylenchus } \\
\text { sp.3 }\end{array}$ & $\begin{array}{l}\text { USA, } \\
\text { Florida }\end{array}$ & $\begin{array}{l}\text { Unknown } \\
\text { plant }\end{array}$ & CD2726 & MW413573 & - & - & MW411820 & $\begin{array}{c}\text { S.A. } \\
\text { Subbotin }\end{array}$ \\
\hline $\begin{array}{l}\text { Paratylenchus } \\
\text { sp. } 4\end{array}$ & $\begin{array}{l}\text { USA, } \\
\text { Oregon }\end{array}$ & Trees & CD986 & KF242203 & - & - & MW411829 & $\begin{array}{c}\text { S.A. } \\
\text { Subbotin, } \\
\text { Van den Berg } \\
\text { et al. [22] }\end{array}$ \\
\hline $\begin{array}{l}\text { Paratylenchus } \\
\text { sp.7 }\end{array}$ & $\begin{array}{c}\text { USA, } \\
\text { California, } \\
\text { Riverside, } \\
\text { UCR campus }\end{array}$ & $\begin{array}{l}\text { Unknown } \\
\text { plant }\end{array}$ & CD1004 & KF242242 & - & - & MW411830 & $\begin{array}{c}\text { S.A. } \\
\text { Subbotin }\end{array}$ \\
\hline $\begin{array}{l}\text { Paratylenchus } \\
\text { sp.AH }\end{array}$ & $\begin{array}{c}\text { USA, } \\
\text { California, } \\
\text { El Dorado } \\
\text { county, } \\
\text { Placerville, } \\
\text { Apple hills, } \\
\text { sample N12 }\end{array}$ & $\begin{array}{l}\text { Unknown } \\
\text { plant }\end{array}$ & CD1692 & MW420921 & - & - & MW411844 & $\begin{array}{c}\text { S.A. } \\
\text { Subbotin }\end{array}$ \\
\hline $\begin{array}{l}\text { Paratylenchus } \\
\text { sp.BE11 }\end{array}$ & $\begin{array}{c}\text { Belgium, } \\
\text { Zwijnaarde; } \\
51^{\circ} 00^{\prime} 19^{\prime \prime} \mathrm{N} ; \\
3^{\circ} 42^{\prime} 11^{\prime \prime} \mathrm{E}\end{array}$ & $\begin{array}{l}\text { Grasses } \\
\text { under } \\
\text { a tree }\end{array}$ & BE11 & $\begin{array}{l}\text { MW413672- } \\
\text { MW413674 }\end{array}$ & MW413617 & - & $\begin{array}{l}\text { MW421687- } \\
\text { MW421688 }\end{array}$ & $\begin{array}{c}\text { C.M. } \\
\text { Etongwe }\end{array}$ \\
\hline $\begin{array}{l}\text { Paratylenchus } \\
\text { sp.CaD }\end{array}$ & $\begin{array}{l}\text { USA, } \\
\text { California, } \\
\text { El Dorado } \\
\text { county, } \\
\text { Placerville, } \\
\text { Apple hills, } \\
\text { sample, N7 }\end{array}$ & $\begin{array}{l}\text { Unknown } \\
\text { plant }\end{array}$ & CD1686 & MW413561 & - & - & MW411841 & $\begin{array}{c}\text { S.A. } \\
\text { Subbotin }\end{array}$ \\
\hline
\end{tabular}


Table 1. Cont.

\begin{tabular}{|c|c|c|c|c|c|c|c|c|}
\hline \multirow[b]{2}{*}{ Species } & \multirow[b]{2}{*}{ Locality } & \multirow{2}{*}{$\begin{array}{l}\text { Associated } \\
\text { Plant Host }\end{array}$} & \multirow{2}{*}{$\begin{array}{l}\text { Sample } \\
\text { Code }\end{array}$} & \multicolumn{4}{|c|}{ GenBank Accession Numbers } & \multirow[b]{2}{*}{ Source } \\
\hline & & & & $\begin{array}{l}\text { 28S } \\
\text { rRNA }\end{array}$ & $\begin{array}{l}\text { ITS } \\
\text { rRNA }\end{array}$ & $\begin{array}{c}\text { 18S } \\
\text { rRNA }\end{array}$ & $\begin{array}{l}\text { COI of } \\
\text { mtDNA }\end{array}$ & \\
\hline $\begin{array}{l}\text { Paratylenchus } \\
\text { sp.CaD }\end{array}$ & $\begin{array}{c}\text { USA, } \\
\text { California, } \\
\text { El Dorado } \\
\text { county, } \\
\text { Placerville, } \\
\text { Apple hills, } \\
\text { sample N16 }\end{array}$ & $\begin{array}{l}\text { Unknown } \\
\text { plant }\end{array}$ & CD1695a, b & $\begin{array}{l}M W 413560 \\
M W 413562\end{array}$ & MW413584 & - & MW411824 & $\begin{array}{c}\text { S.A. } \\
\text { Subbotin }\end{array}$ \\
\hline $\begin{array}{l}\text { Paratylenchus } \\
\text { sp.CaD }\end{array}$ & $\begin{array}{c}\text { USA, } \\
\text { California, } \\
\text { El Dorado } \\
\text { county, } \\
\text { Placerville, } \\
\text { Apple hills, } \\
\text { sample N20 }\end{array}$ & $\begin{array}{l}\text { Unknown } \\
\text { plant }\end{array}$ & CD1696 & MW413563 & - & - & - & $\begin{array}{c}\text { S.A. } \\
\text { Subbotin }\end{array}$ \\
\hline $\begin{array}{l}\text { Paratylenchus } \\
\text { sp.CaD }\end{array}$ & $\begin{array}{c}\text { USA, } \\
\text { California, } \\
\text { Yolo county, } \\
\text { Putah Creek }\end{array}$ & Rubus sp. & CD1791 & MW413559 & - & - & - & $\begin{array}{c}\text { S.A. } \\
\text { Subbotin }\end{array}$ \\
\hline $\begin{array}{l}\text { Paratylenchus } \\
\text { sp.D }\end{array}$ & $\begin{array}{l}\text { Belgium, } \\
\text { Ghent, } \\
\text { Blaarmeersen; } \\
51^{\circ} 02^{\prime} 14^{\prime \prime} \mathrm{N} ; \\
3^{\circ} 41^{\prime} 23^{\prime \prime} \mathrm{E}\end{array}$ & $\begin{array}{l}\text { Grasses } \\
\text { under } \\
\text { a tree }\end{array}$ & BE20 & $\begin{array}{l}\text { MW413664- } \\
\text { MW413669 }\end{array}$ & $\begin{array}{l}\text { MW413611- } \\
\text { MW413614 }\end{array}$ & $\begin{array}{l}\text { MW413719- } \\
M W 413723\end{array}$ & $\begin{array}{l}\text { MW421689- } \\
\text { MW421699 }\end{array}$ & $\begin{array}{c}\text { C.M. } \\
\text { Etongwe }\end{array}$ \\
\hline $\begin{array}{l}\text { Paratylenchus } \\
\text { sp.Dia }\end{array}$ & $\begin{array}{c}\text { USA, } \\
\text { California, } \\
\text { Contra Costa } \\
\text { County, } \\
\text { Mount } \\
\text { Diablo } \\
\text { State Park }\end{array}$ & $\begin{array}{l}\text { Unknown } \\
\text { plant }\end{array}$ & CD1776 & MW413574 & - & - & MW411833 & $\begin{array}{c}\text { S.A. } \\
\text { Subbotin }\end{array}$ \\
\hline $\begin{array}{l}\text { Paratylenchus } \\
\text { sp.F }\end{array}$ & $\begin{array}{l}\text { Belgium, } \\
\text { Ghent, } \\
\text { Blaarmeersen; } \\
51^{\circ} 07^{\prime} 14^{\prime \prime} \mathrm{N} ; \\
2^{\circ} 39^{\prime} 29^{\prime \prime} \mathrm{E}\end{array}$ & $\begin{array}{l}\text { Grasses } \\
\text { under } \\
\text { a tree }\end{array}$ & BE18 & - & - & MW413728 & - & $\begin{array}{c}\text { C.M. } \\
\text { Etongwe }\end{array}$ \\
\hline $\begin{array}{l}\text { Paratylenchus } \\
\text { sp.F }\end{array}$ & $\begin{array}{l}\text { Belgium, } \\
\text { Merendree; } \\
51^{\circ} 04^{\prime} 12^{\prime \prime} \mathrm{N} ; \\
3^{\circ} 34^{\prime} 37^{\prime \prime} \mathrm{E}\end{array}$ & $\begin{array}{l}\text { Grasses } \\
\text { around a } \\
\text { beech tree }\end{array}$ & BE22 & $\begin{array}{l}\text { MW413675- } \\
M W 413679\end{array}$ & $\begin{array}{l}\text { MW413618- } \\
\text { MW413620 }\end{array}$ & $\begin{array}{c}\text { MW413727, } \\
\text { MW413729- } \\
\text { MW413731 }\end{array}$ & $\begin{array}{l}\text { MW421700- } \\
\text { MW421702 }\end{array}$ & $\begin{array}{c}\text { C.M. } \\
\text { Etongwe }\end{array}$ \\
\hline $\begin{array}{l}\text { Paratylenchus } \\
\text { sp.F }\end{array}$ & $\begin{array}{c}\text { Russia, } \\
\text { Primorsky } \\
\text { Krai, } \\
\text { Olginsky } \\
\text { district }\end{array}$ & $\begin{array}{l}\text { Unknown } \\
\text { plant }\end{array}$ & $\begin{array}{l}\text { CD1842, } \\
\text { CD1844 }\end{array}$ & $\begin{array}{l}\text { MW413571, } \\
\text { MW413572 }\end{array}$ & - & & - & J. Zograf \\
\hline $\begin{array}{l}\text { Paratylenchus } \\
\text { sp.Ge16 }\end{array}$ & $\begin{array}{c}\text { Ethiopia, } \\
\text { Jimma Zone, } \\
\text { Gera district }\end{array}$ & Coffee & Ge16c & $\begin{array}{c}\text { MW413680- } \\
\text { MW413682 }\end{array}$ & - & $\begin{array}{l}\text { MW413732- } \\
\text { MW413734 }\end{array}$ & $\begin{array}{l}\text { MW421703- } \\
\text { MW421705 }\end{array}$ & $\begin{array}{c}\text { C.M. } \\
\text { Etongwe }\end{array}$ \\
\hline $\begin{array}{c}\text { Paratylenchus } \\
\text { sp.J }\end{array}$ & $\begin{array}{c}\text { USA, } \\
\text { Washington, } \\
\text { Mason } \\
\text { County, } \\
\text { Skokomish, } \\
\text { sample 32E; } \\
47^{\circ} 18^{\prime} 07.0^{\prime \prime} \mathrm{N} \\
123^{\circ} 10^{\prime} 95.6^{\prime \prime} \mathrm{W}\end{array}$ & $\begin{array}{l}\text { Unknown } \\
\text { plant }\end{array}$ & CD3216 & MW413570 & - & - & - & $\begin{array}{c}\text { S.A. } \\
\text { Subbotin }\end{array}$ \\
\hline
\end{tabular}


Table 1. Cont.

\begin{tabular}{|c|c|c|c|c|c|c|c|c|}
\hline \multirow[b]{2}{*}{ Species } & \multirow[b]{2}{*}{ Locality } & \multirow{2}{*}{$\begin{array}{l}\text { Associated } \\
\text { Plant Host }\end{array}$} & \multirow{2}{*}{$\begin{array}{l}\text { Sample } \\
\text { Code }\end{array}$} & \multicolumn{4}{|c|}{ GenBank Accession Numbers } & \multirow[b]{2}{*}{ Source } \\
\hline & & & & $\begin{array}{c}28 S \\
\text { rRNA }\end{array}$ & $\begin{array}{c}\text { ITS } \\
\text { rRNA }\end{array}$ & $\begin{array}{c}\text { 18S } \\
\text { rRNA }\end{array}$ & $\begin{array}{l}\text { COI of } \\
\text { mtDNA }\end{array}$ & \\
\hline $\begin{array}{l}\text { Paratylenchus } \\
\text { sp.J }\end{array}$ & $\begin{array}{c}\text { USA, } \\
\text { Oregon, } \\
\text { Douglas } \\
\text { County, } \\
\text { Oakland, } \\
\text { sample 35; } \\
43^{\circ} 28^{\prime} 59.9^{\prime \prime} \mathrm{N} \\
123^{\circ} 19^{\prime} 24.5^{\prime \prime} \mathrm{W} \\
\end{array}$ & $\begin{array}{l}\text { Unknown } \\
\text { plant }\end{array}$ & CD3220 & MW413569 & - & - & - & $\begin{array}{c}\text { S.A. } \\
\text { Subbotin }\end{array}$ \\
\hline $\begin{array}{l}\text { Paratylenchus } \\
\text { sp.NL }\end{array}$ & $\begin{array}{l}\text { The } \\
\text { Netherlands, } \\
\text { Hilversum }\end{array}$ & Holly & NL & $\begin{array}{l}\text { MW413683- } \\
\text { MW413684 }\end{array}$ & - & $\begin{array}{l}\text { MW413740- } \\
\text { MW413742 }\end{array}$ & $\begin{array}{l}\text { MW421706- } \\
\text { MW421707 }\end{array}$ & G. Karssen \\
\hline $\begin{array}{l}\text { Paratylenchus } \\
\text { sp.SK }\end{array}$ & South Korea & Pinus sp. & CD1384 & MW413580 & - & & - & $\begin{array}{c}\text { S.A. } \\
\text { Subbotin }\end{array}$ \\
\hline
\end{tabular}

\subsubsection{Paratylenchus aculentus}

Females (Sample BE9; Figure 1, Table 2): Heat relaxed specimens open C- to J-shape. Lateral field with three lateral lines. Deirids not observed (not necessarily an indication that they are absent). Cephalic region rounded, low, sometimes appearing slightly truncated, submedian lobes not protruded. Stylet 52-61 $\mu \mathrm{m}$ long, cone $80-91 \%$ of stylet length, knobs 2-4 $\mu \mathrm{m}$ across. Pharynx well developed, about one-third of body length. Secretoryexcretory pore between median bulb and isthmus level. Spermatheca rounded to slightly oval and filled with sperm cells. Prevulval swelling not prominent. Vulval flaps very small and can be visible under LM. Vulval located at $71-76 \%$ of body length from anterior end. Vagina straight to slightly oblique, reaching to almost half of body width. Anus obscure. Tail 18-25 $\mu \mathrm{m}$ long, tapers gradually to a finely or bluntly rounded terminus.

Molecular characterisation: Three D2-D3 of 28S, two ITS, two 18S rRNA and four COI gene sequences were generated without intraspecific sequence variations. The D2-D3 of $28 \mathrm{~S}$ and the $18 \mathrm{~S}$ sequences, respectively, were found to be similar to KP966492 (99\% similarity; 4 out of 544 bp difference) and KP966494 (100\% similarity; 800 bp) of P. colinus from Iran after Hesar et al. [24].

Remarks: Males were not found. Female morphology and morphometrics matched very well with P. aculentus. This species has been reported earlier in Belgium [53]. Although the D2-D3 and 18S sequences pointed towards P. colinus, the current population had no cuticular ornamentations present in the anterior part of the body and female bodies were not swollen and submedian lobe protrusions were not seen, which are important characteristics for P. colinus. According to Ghaderi et al. [23], P. aculentus is part of Group 9 of the Paratylenchus species with stylet lengths longer than $40 \mu \mathrm{m}$, three lateral lines and absence of vulval flaps. Here, we confirm the presence of small vulval flaps in $P$. aculentus, clearly supported by SEM. This was also an observation originally made by Brzeski [19]. Paratylenchus aculentus should, therefore, be placed in Group 8 with P. colinus and P. idalimus; furthermore, the close affinity of our P. aculentus population with P. colinus is also molecularly supported by the very conserved $18 \mathrm{~S}$ rRNA gene fragment. 

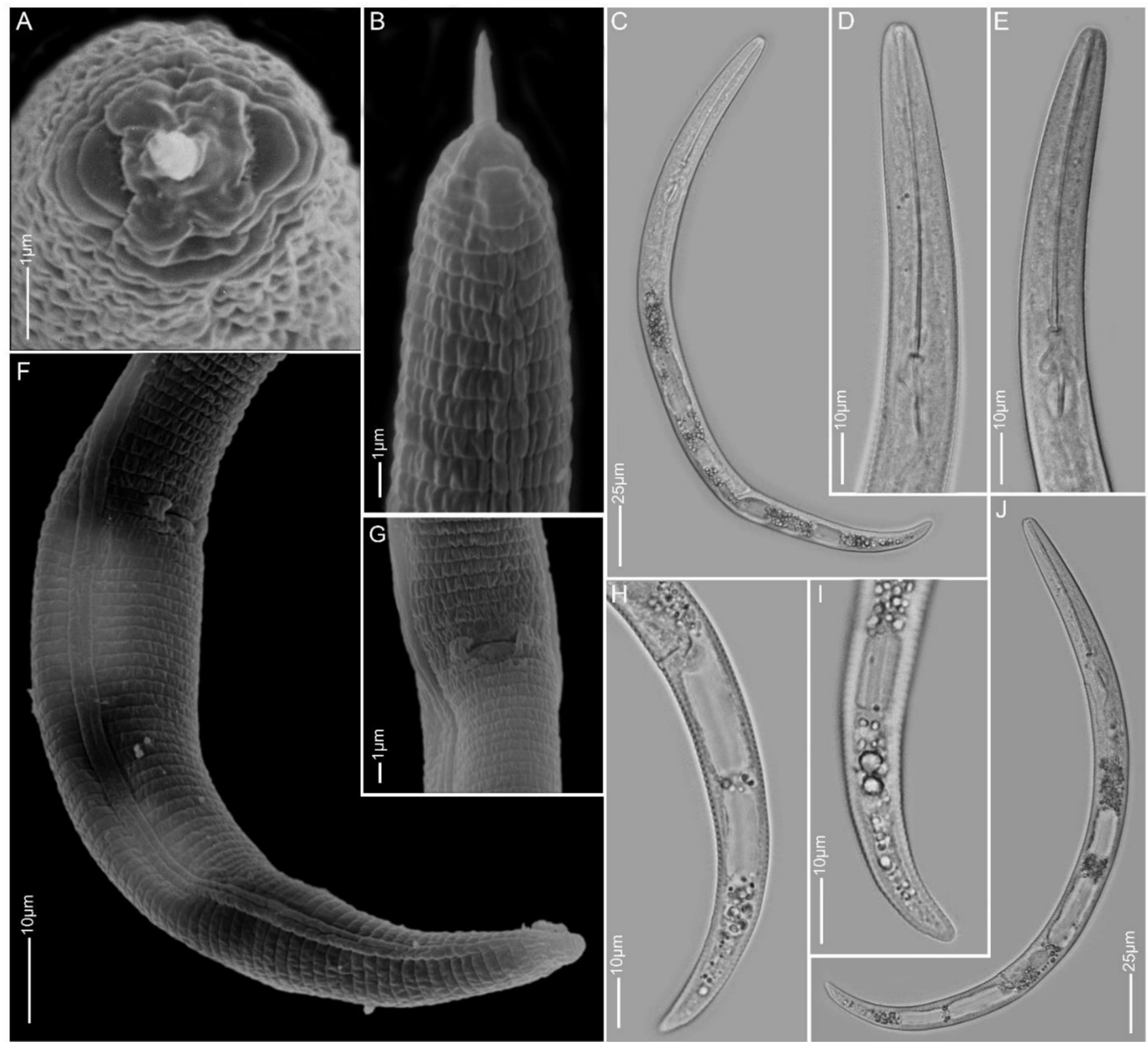

Figure 1. Light and scanning electron microscopy images of Paratylenchus aculentus females: (A) face view; (B,D,E) anterior region; $(\mathbf{C}, \mathbf{J})$ total body; $(\mathbf{G})$ vulva region; $(\mathbf{F}, \mathbf{H}, \mathbf{I})$ tail region. 
Table 2. Female morphometrics of Paratylenchus aculentus, Paratylenchus goodeyi, Paratylenchus idalimus and Paratylenchus straeleni from fixed specimens mounted in glycerine. All measurements except for ratios and percentages are given in $\mu \mathrm{m}$ and in the form mean \pm stdev (range).

\begin{tabular}{ccccc}
\hline Population & $\begin{array}{c}\text { P. aculentus } \\
\text { (BE9) }\end{array}$ & $\begin{array}{c}\text { P. goodeyi } \\
\text { (BE22) }\end{array}$ & $\begin{array}{c}\text { P. idalimus } \\
\text { (BE19 and BE20) }\end{array}$ & $\begin{array}{c}\text { P. straeleni } \\
\text { (BE15) }\end{array}$ \\
\hline $\mathrm{n}$ & 12 & 17 & 7 & 11 \\
$\mathrm{~L}$ & $266 \pm 20.1(233-03)$ & $348 \pm 42.5(266-452)$ & $299 \pm 20.7(278-332)$ & $358 \pm 13.1(330-379)$ \\
$\mathrm{a}$ & $19.6 \pm 2.0(16.3-23.2)$ & $20.7 \pm 1.7(16.7-23.2)$ & $21.0 \pm 1.3(20-23)$ & $22.6 \pm 0.9(20.7-24.3)$ \\
$\mathrm{b}$ & $2.6 \pm 0.1(2.4-2.8)$ & $3.2 \pm 0.3(2.9-3.7)$ & $2 \pm 0.2(2.0-2.4)$ & $3.6 \pm 0.1(3.4-3.7)$ \\
$\mathrm{c}$ & $12.4 \pm 1.5(10.8-15.2)$ & $11.9 \pm 1.4(10.1-13.5)$ & $12.1 \pm 0.6(12.1-13.1)$ & $10.6 \pm 1.0(8.9-11.8)$ \\
$\mathrm{c}$ & $2.8 \pm 0.3(2.4-3.1)$ & $3.0 \pm 0.2(2.8-3.3)$ & $4.0 \pm 0.6(3.1-4.1)$ & $3.4 \pm 0.3(3.0-3.9)$ \\
Maximum body width & $13.6 \pm 1.3(11.6-15.5)$ & $17.0 \pm 3.3(13.0-27.0)$ & $14.0 \pm 1.4(13.0-16.1)$ & $15.9 \pm 0.7(14.6-16.7)$ \\
Stylet length & $56.0 \pm 3.3(52.4-61.2)$ & $52.1 \pm 2.8(47.0-58.6)$ & $89.0 \pm 3.5(84.1-93.0)$ & $55.7 \pm 1.7(53.5-58.6)$ \\
Cone length & $49.1 \pm 3.6(43.0-54.9)$ & $43.0 \pm 2.7(48.2-48.5)$ & $78.0 \pm 2.9(74.0-83.1)$ & $44.7 \pm 1.7(42.2-47.4)$ \\
Cone\%stylet & $87.5 \pm 3.8(80.1-91.0)$ & $82.4 \pm 2.5(78.0-89.6)$ & $88.0 \pm 2.2(83-89)$ & $80 \pm 1.8(76-83)$ \\
Knob width & $3.2 \pm 0.5(2.3-4.0)$ & $4.1 \pm 0.6(3.3-5.2)$ & $4.0 \pm 0.2(4.0-4.3)$ & $3.9 \pm 0.4(3.1-4.6)$ \\
Pharynx length & $101 \pm 8.3(87.0-113)$ & $109 \pm 11.7(92.7-133)$ & $130 \pm 12.8(114-147)$ & $100 \pm 3.8(92.1-105)$ \\
Anterior end to SE pore & $66.7 \pm 5.2(54.3-74.4)$ & $80.7 \pm 8.6(68.5-99.0)$ & $93.0 \pm 11.7(82.0-115)$ & $82.5 \pm 2.9(79.4-87.6)$ \\
SE pore\%L & $25.2 \pm 0.9(23.3-26.4)$ & $22.9 \pm 1.4(21.0-25.8)$ & $31 \pm 4.2(28-40)$ & $23 \pm 0.7(22-24)$ \\
Anterior end to vulva & $193 \pm 16.1(165-218)$ & $279 \pm 31.9(216-356)$ & $233 \pm 17.9(214-260)$ & $270 \pm 18.8(249-330)$ \\
V\% & $72.5 \pm 1.5(70.8-75.7)$ & $80.1 \pm 1.4(77.8-82.3)$ & $78.0 \pm 0.9(77-79)$ & $81 \pm 1.9(80-84)$ \\
Body width at anus & $7.6 \pm 0.5(7.0-8.3)$ & $9.7 \pm 0.5(9.7-10.0)$ & $7.0 \pm 0.1(7.0-7.1)$ & $10.1 \pm 0.4(9.1-10.5)$ \\
Tail length & $20.9 \pm 2.3(18.1-25.1)$ & $29.0 \pm 2.4(25.8-32.1)$ & $25.0 \pm 2.2(22.0-28.1)$ & $34.4 \pm 3.7(31-40.8)$ \\
\hline
\end{tabular}

\subsubsection{Paratylenchus elachistus}

Females (Sample BE15; Figure 2, Table 3): Heat relaxed specimens open C- to J-shape. Lateral field with four lateral lines. Deirids visible under SEM. Cephalic region conicalrounded to sometimes truncated. En face square-shaped, showing poorly developed submedian lobes, two pronounced lateral ridges and small indistinct dorso-ventral ridges around oral opening, two slit-like amphidial openings laterally. Stylet 20-22 $\mu \mathrm{m}$ long, cone $61-68 \%$ of stylet length and knobs $3-4 \mu \mathrm{m}$ across. Pharynx well developed, about one-fourth of body length. Hemizonid commonly above secretory-excretory pore about two body annuli long. Secretory-excretory pore between mid-isthmus and end bulb level. Spermatheca rounded to oval and filled with sperm cells. Vulval flaps rounded, prominent. Vulva located at $80-83 \%$ of body length from anterior end. Vagina oblique, reaching to half of body width. Tail 21-29 $\mu \mathrm{m}$ long, conical, thin and terminus from spicate to pointed or minutely rounded.

Molecular characterisation: Two D2-D3 of 28S, four ITS, four 18S rRNA and four COI gene sequences were generated for the first time from this species without intraspecific sequence variations.

Remarks: Males were not found. This species is reported for the first time in Belgium and has only been recorded in Poland and Slovakia before in Europe $[19,54,55]$. Female morphology and morphometrics agree well with the original description [56] and also with descriptions of other populations [19,31]. Paratylenchus elachistus can be separated from its closest species, Paratylenchus minutus, Lindford in Lindford, Oliveira \& Ishii, 1949, by a longer body length $(0.23-0.34 \mathrm{~mm}$ vs. $0.19-0.31 \mathrm{~mm})$, a more robust and longer stylet (19-25 $\mu \mathrm{m}$ vs. $15-21 \mu \mathrm{m})$ and a more slender tail, commonly with spicate to finely rounded tail termini. 

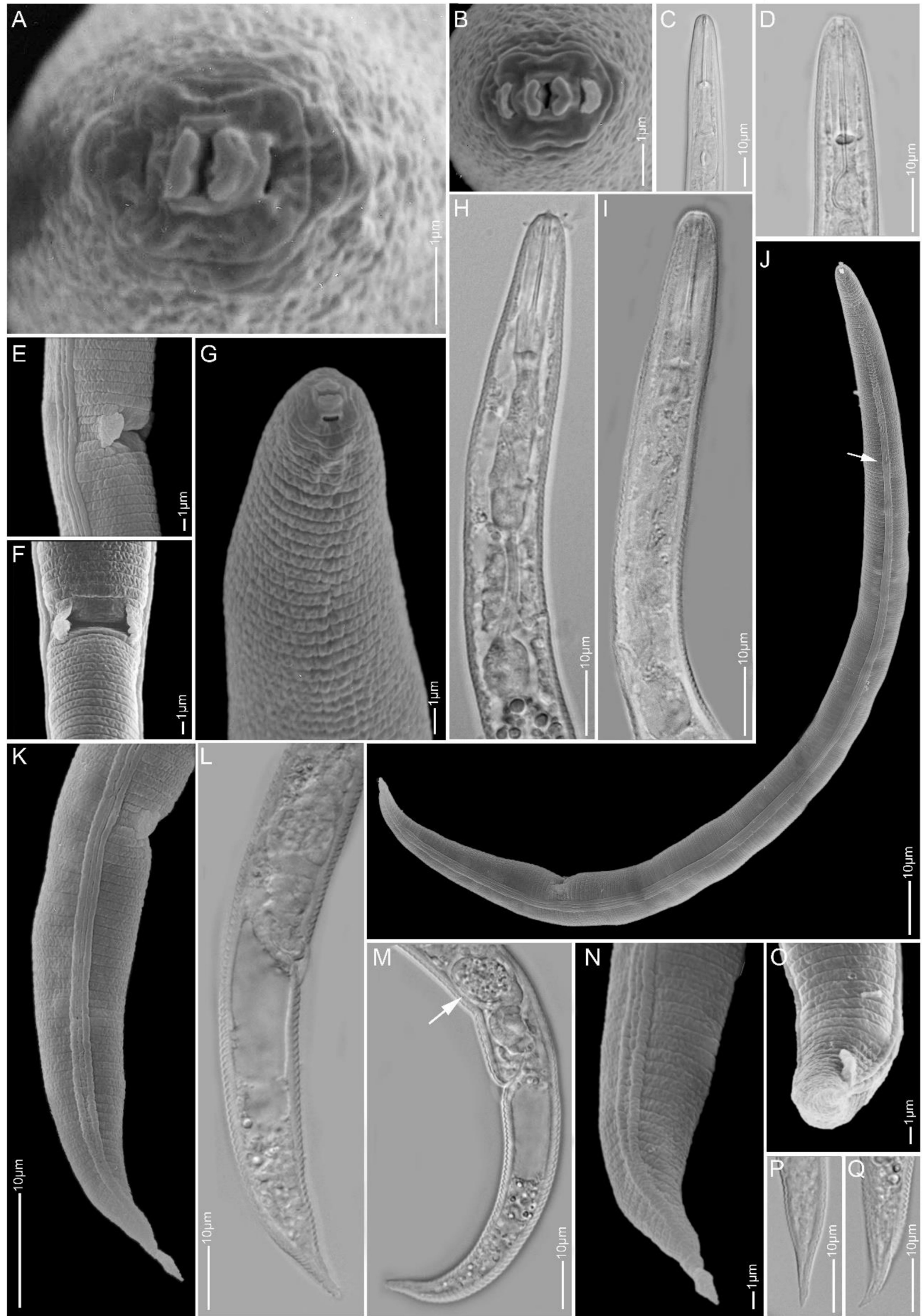

Figure 2. Light and scanning electron microscopy images of Paratylenchus elachistus females: (A,B) face view; (C,D,G-I) anterior region; $(\mathbf{E}, \mathbf{F})$ vulva region; $(\mathbf{J})$ total body; $(\mathbf{K}-\mathbf{Q})$ tail region; arrows pointed to deirid in $(\mathbf{J})$ and spermatheca in $(\mathbf{M})$. 
Table 3. Female morphometrics of Paratylenchus elachistus, Paratylenchus holdemani, Paratylenchus microdorus and Paratylenchus veruculatus from fixed specimens mounted in glycerine. All measurements except for ratios and percentages are given in $\mu \mathrm{m}$ and in the form mean \pm stdev (range).

\begin{tabular}{ccccc}
\hline Population & $\begin{array}{c}\text { P. elachistus } \\
\text { (BE15) }\end{array}$ & $\begin{array}{c}\text { P. holdemani } \\
\text { (AR3) }\end{array}$ & $\begin{array}{c}\text { P. microdorus } \\
\text { (BE9) }\end{array}$ & $\begin{array}{c}\text { P. veruculatus } \\
\text { (BE20) }\end{array}$ \\
\hline $\mathrm{n}$ & 24 & 31 & 10 & 15 \\
$\mathrm{~L}$ & $301 \pm 12.5(283-329)$ & $359 \pm 47(285-475)$ & $330.7 \pm 20(297-355)$ & $286 \pm 24.7(251-331)$ \\
$\mathrm{a}$ & $20.4 \pm 1.1(17.7-22.6)$ & $20.9 \pm 1.9(16.4-25.2)$ & $21.4 \pm 1.4(18.7-23.1)$ & $19.8 \pm 1.9(17.2-23.3)$ \\
$\mathrm{b}$ & $4.1 \pm 0.2(3.8-4.3)$ & $4.1 \pm 0.7(2.2-5.1)$ & $5.0 \pm 0.5(4.5-6.2)$ & $3.8 \pm 0.3(3.3-4.2)$ \\
$\mathrm{c}$ & $12.1 \pm 0.9(10.9-14.3)$ & $14.8 \pm 1.4(12.4-17.7)$ & $10.6 \pm 0.9(9.2-12)$ & $17.8 \pm 1.8(14.6-20.6)$ \\
$\mathrm{c}^{\prime}$ & $2.8 \pm 0.2(2.4-3.3)$ & $2.5 \pm 0.3(2.1-3.2)$ & $3.8 \pm 0.5(2.8-4.5)$ & $2.2 \pm 0.2(1.8-2.6)$ \\
Stylet length & $14.8 \pm 1.0(12.7-16.6)$ & $17.3 \pm 3.0(11.3-23.8)$ & $15.5 \pm 1.4(13.3-17.1)$ & $14.5 \pm 1.4(12.5-16.5)$ \\
Cone length & $13.3 \pm 0.4(19.7-22.2)$ & $22.5 \pm 2.0(19.0-26.1)$ & $12.4 \pm 1.3(10.6-14.7)$ & $14.2 \pm 0.5(13.1-14.8)$ \\
Cone\%stylet & $63.7 \pm 1.5(60.5-67.5)$ & $67.3 \pm 3.5(60.9-77.4)$ & $53.4 \pm 5.7(45.3-60.4)$ & $62.8 \pm 1.1(60.3-64.8)$ \\
Knob width & $3.5 \pm 0.2(3.1-4.1)$ & $3.3 \pm 0.4(2.9-4.2)$ & & $-6.9 \pm 0.3(8.3-9.3)$ \\
Pharynx length & $74.5 \pm 2.6(70.4-80.6)$ & $89.7 \pm 21.5(66.1-161)$ & $66.6 \pm 6.4(56.2-76.1)$ & $75.8 \pm 7.0(60.8-88.4)$ \\
Anterior end to SE pore & $60.7 \pm 3.9(54.0-68.5)$ & $74.8 \pm 9.1(60.1-99.0)$ & $63.6 \pm 4.7(57.5-71.3)$ & $62.3 \pm 6.5(51.2-74.4)$ \\
SE pore\%L & $20.1 \pm 0.9(18.6-21.9)$ & $21.2 \pm 1.8(16.4-23.7)$ & $19.2 \pm 1.1(17.3-20.6)$ & $21.8 \pm 2.0(17.5-25.6)$ \\
Anterior end to vulva & $245 \pm 10.2(226-269)$ & $303 \pm 40.9(238-391)$ & & - \\
V\% & $81.3 \pm 0.9(79.7-83.2)$ & $84.3 \pm 1.8(81.3-90.5)$ & $81 \pm 1.6(79.1-82.8)$ & $85.7 \pm 1.4(83.8-89.7)$ \\
Body width at anus & $8.9 \pm 0.7(7.5-9.9)$ & $10.0 \pm 1.3(7.2-12.3)$ & $8.5 \pm 1.3(6.8-10.8)$ & $7.3 \pm 0.6(6.3-8.8)$ \\
Tail length & $24.8 \pm 2.2(20.9-29.1)$ & $25.2 \pm 2.8(20.0-29.5)$ & $31.8 \pm 3.1(28.1-35.9)$ & $16.1 \pm 1.7(13.6-19.1)$ \\
\hline
\end{tabular}

\subsubsection{Paratylenchus goodeyi}

Females (Sample BE22; Figure 3, Table 2): Heat relaxed specimens C- to J-shape. Lateral field with four lateral lines. Deirids observed under LM. Cephalic region conical-rounded, submedian lobes not protruding except in two freshly killed specimens where small protrusions were seen under LM. Stylet $47-59 \mu \mathrm{m}$ long, cone $78-90 \%$ of stylet length, stylet guide faintly seen, knobs 3-5 $\mu$ m across. Pharynx well developed, about one-third of body length. Secretory-excretory pore around median bulb level. Spermatheca oval to elongate, filled with sperm cells. Vulval flaps present. Vulva located at $78-82 \%$ of body length from anterior end, in one female a short post-vulva sac observed. Vagina oblique and reaching to two-third of body width. Tail $26-32 \mu \mathrm{m}$ long, conoid with variable terminus from finely rounded to bluntly rounded and rarely pointed.

Molecular characterisation: Three D2-D3 of 28S, one ITS, two 18S rRNA and three COI gene sequences were generated for the first time for this species without intraspecific sequence variations.

Remarks: Males were not found. Females morphology and morphometrics agree well with former $P$. goodeyi descriptions $[18,19,57]$. This species was originally described from the Netherlands and has been reported from many European countries, including Belgium. Paratylenchus goodeyi is one of the 22 species of the Group 10 of Paratylenchus after Ghaderi et al. [23] with stylet length more than $40 \mu \mathrm{m}$, four lateral lines and presence of vulval flaps. This species is comparable to other members of the group namely Paratylenchus ivorensis Luc \& de Guiran, 1962, Paratylenchus pandatus (Raski, 1976) Siddiqi, 1989 and P. straeleni with females having more or less conical-rounded heads, stylet lengths in the range $40-61 \mu \mathrm{m}$ (except for $P$. pandatus for which, a stylet length up to $68 \mu \mathrm{m}$ was rarely reported). However, the vulvae of $P$. goodeyi and P. straeleni are located more posteriorly (77-88\%) than that of the other two species (70-78\%); P. ivorensis and P. goodeyi have been reported with variable tail termini, while $P$. pandatus and $P$. straeleni have been found usually with finely rounded to sub-acute female tail termini. 


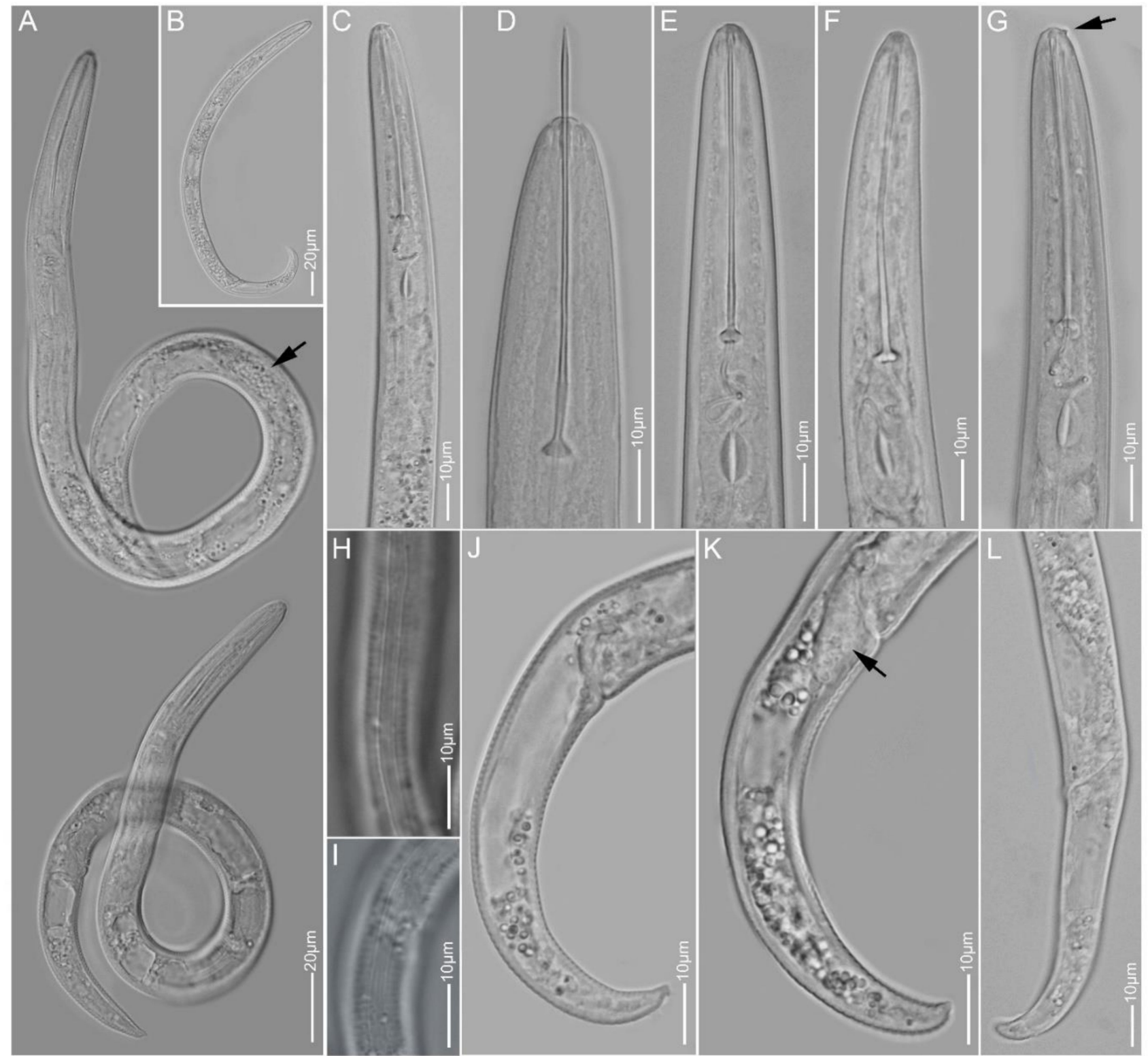

Figure 3. Light microscopy images of Paratylenchus goodeyi females: $(\mathbf{A}, \mathbf{B})$ total body; $(\mathbf{C}-\mathbf{G})$ anterior region; $(\mathbf{H}, \mathbf{I})$ lateral field; (J-L) tail region; arrows pointed to spermatheca in A, protruding submedian lobe in $\mathrm{G}$ and post-vulva sac in $\mathrm{K}$.

\subsubsection{Paratylenchus holdemani}

Females (Sample AR3; Figure 4, Table 3): Heat relaxed specimens C- to J-shape. Lateral field with four lateral lines. Deirids not observed. Cephalic region slightly truncated, submedian lobes not protruded. En face showing four small submedian lobes, four irregular ridges around oral opening, slit-like lateral amphidial openings. Stylet 19-26 $\mu \mathrm{m}$ long, cone $61-77 \%$ of stylet length, knobs $3-4 \mu \mathrm{m}$ across. Pharynx well developed, about one-fourth of body length. Secretory-excretory pore commonly between mid-isthmus and end bulb level. Spermatheca rounded, filled with sperm cells. Vulval flaps prominent. Vulva located at $81-90 \%$ of body length from anterior end. Vagina oblique and reaching to two-third of body width. Tail 20-30 $\mu \mathrm{m}$ long, conoid with regularly finely rounded to sometimes bluntly rounded or digitate terminus. 

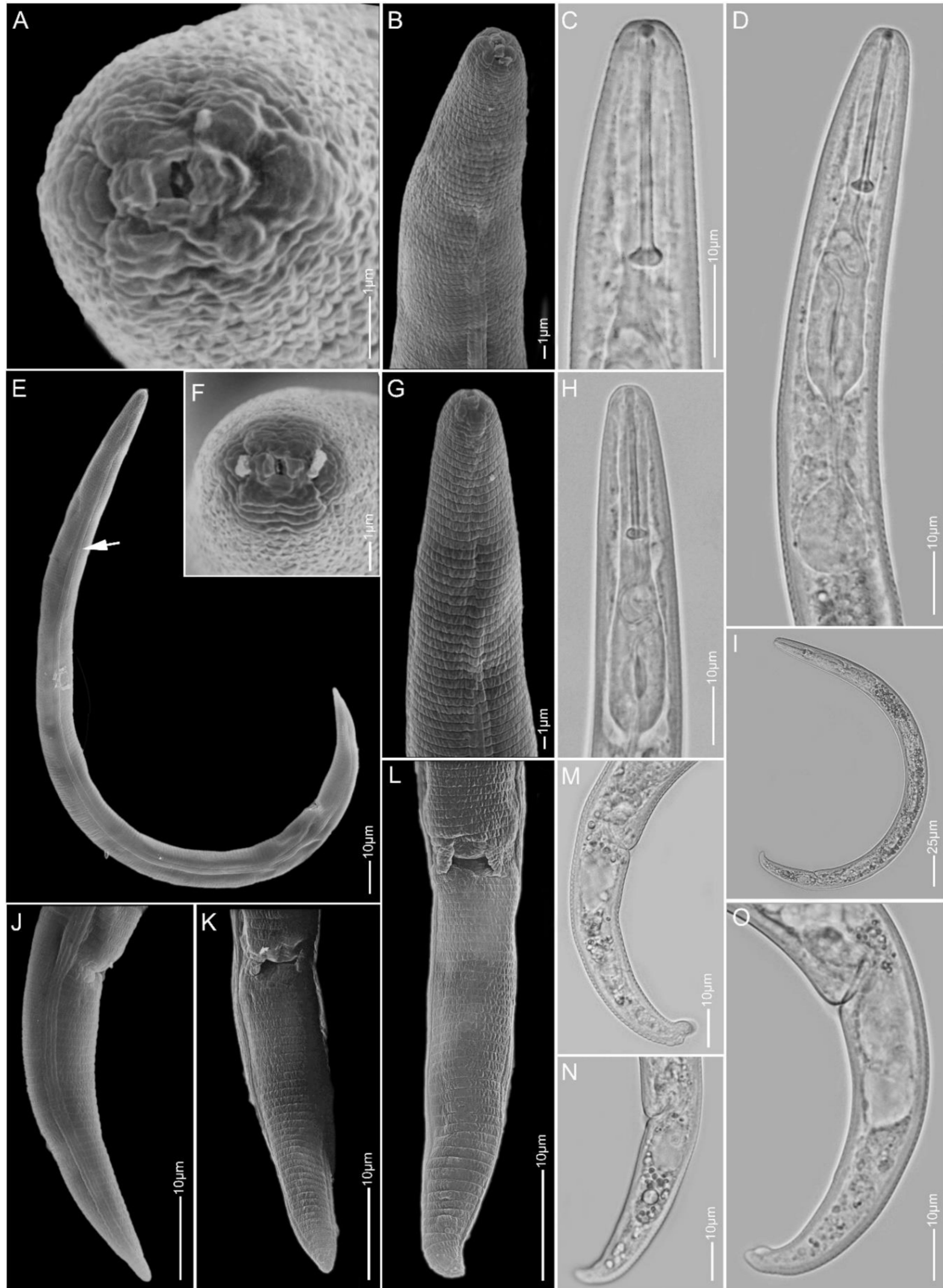

Figure 4. Light and scanning electron microscopy images of Paratylenchus holdemani females: (A,F) face view; (B-D,G,H) anterior region; (E,I) total body; (J-O) tail region; arrow pointed to deirid in (E). 
Males: Two males were obtained from Sample AR3 and one from Sample BE20. Their conspecificity with the females was confirmed by identical D2-D3 of 28S rRNA and COI gene sequences identified from the AR3 and BE20 males, respectively. The males had an average stylet length of approximately $12 \mu \mathrm{m}$ and spicule length of $21 \mu \mathrm{m}$.

Molecular characterisation: Three D2-D3 of 28S, one 18S rRNA and three COI gene sequences were generated from the AR3 females, whereas two D2-D3 of 28S, one ITS, one $18 \mathrm{~S}$ rRNA and four COI gene sequences were generated from the BE20 females. No sequence from either population showed any intraspecific variations. The D2-D3 sequences were found to be identical to P. bukowinensis sequences that originated from Italy [37] and Belgium [47]; however, morphological data for these populations are not available for comparison and both are considered here as representatives of $P$. holdemani.

Remarks: This species has been reported for the first time in Belgium and has only been reported in the Czech Republic in Europe [19]. The morphology and morphometrics of the AR3 population agree well with the original description [58] and with the population from the Czech Republic [19]. Although our D2-D3 sequences were identical to a P. bukowinensis sequence (AY780943), the female morphology of this Belgian population is different from $P$. bukowinensis descriptions.

Most importantly, the average stylet length $(22.5 \mu \mathrm{m})$ of our population is shorter than for many previously reported P. bukowinensis populations. In addition, the cephalic region of $P$. bukowinensis is more rounded than that of P. holdemani. Paratylenchus holdemani is comparable to P. hamatus and Paratylenchus baldaccii Raski, 1975, but is distinguishable from both species by a shorter stylet length of $22.5 \pm 2.0$ (19-26) $\mu \mathrm{m}$ vs. always above $26 \mu \mathrm{m}$. In this study, greater variation in the tail termini was observed in our P. holdemani population compared to the other two species.

\subsubsection{Paratylenchus idalimus}

Females (Samples BE19 and BE20, two nearby localities; Figures 5 and 6, Table 2): Heat relaxed specimens J- or open C-shape. Lateral field with three lateral lines. Deirids not observed. Cephalic region conical-truncate, submedian lobes well developed and protruding. Stylet $84-93 \mu \mathrm{m}$ long of which $83-89 \%$ is cone, prominent stylet guide, knobs about $4 \mu \mathrm{m}$ across. Pharynx well developed, occasionally reaching up to half of body length. Secretory-excretory pore around level of stylet knobs which is above median bulb level. Spermatheca small, rounded, usually filled with sperm cells. Vulval lips slightly protruding. Vulval flaps reduced and small, sometimes easily overlooked. Vulva located at $77-79 \%$ of body length from anterior end. Vagina oblique, often reaching to two-third of body width. Anus obscure. Tail $22-28 \mu \mathrm{m}$ long, conoid with subacute to finely rounded terminus.

Molecular characterisation: Two identical D2-D3 of $28 \mathrm{~S}$ and two identical $18 \mathrm{~S}$ rRNA gene sequences were generated from the BE20 population, whereas one D2-D3 sequence, identical to that of the BE20 sequence, was generated from the BE19 population. These sequences were generated for the first time for this species.

Remarks: Males and swollen females were not found. Only one juvenile was recorded from the BE20 population with a stylet length of $42 \mu \mathrm{m}$. This is the first time the species has been reported in Europe. Female morphology and morphometrics based on seven females from both populations (three from BE19 and four from BE20) agree well with the description of the slender female by Raski [7] in the USA. This species and P. colinus are the only two members of Group 8 of Paratylenchus [23]. It differs from P. colinus in having a longer stylet (84-93 $\mu \mathrm{m}$ vs. 56-72 $\mu \mathrm{m})$, more pronounced protrusion of submedian lobes, slightly posterior position of vulva (77-79\% vs. $69-78 \%$ ) and absence vs. presence of cuticular ornamentation in anterior body. 


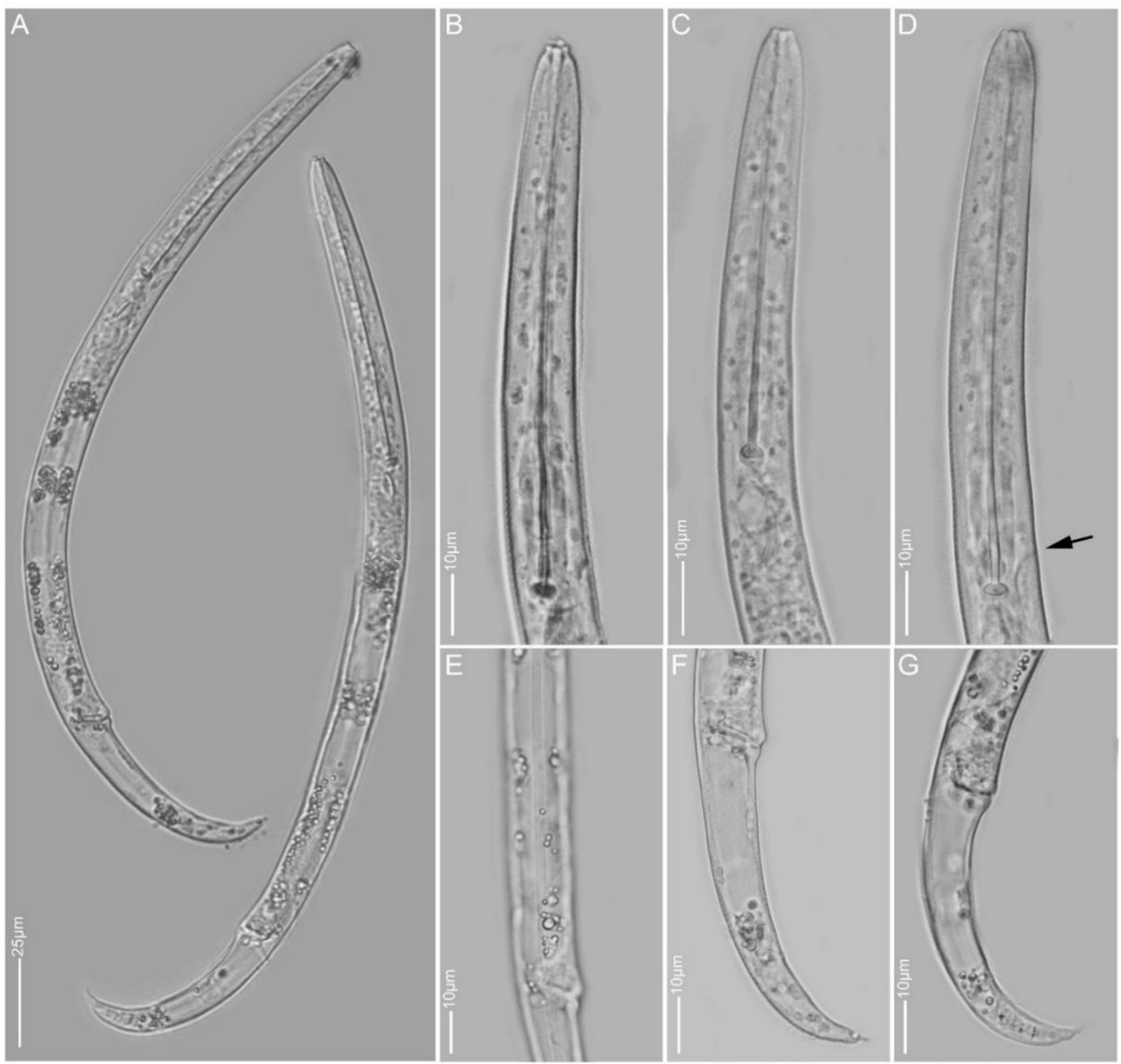

Figure 5. Light microscopy images of Paratylenchus idalimus females from sample BE19: (A) total body; (B-D) anterior region; (E-G) lateral field and tail region; arrow pointed to secretory-excretory pore in D. 

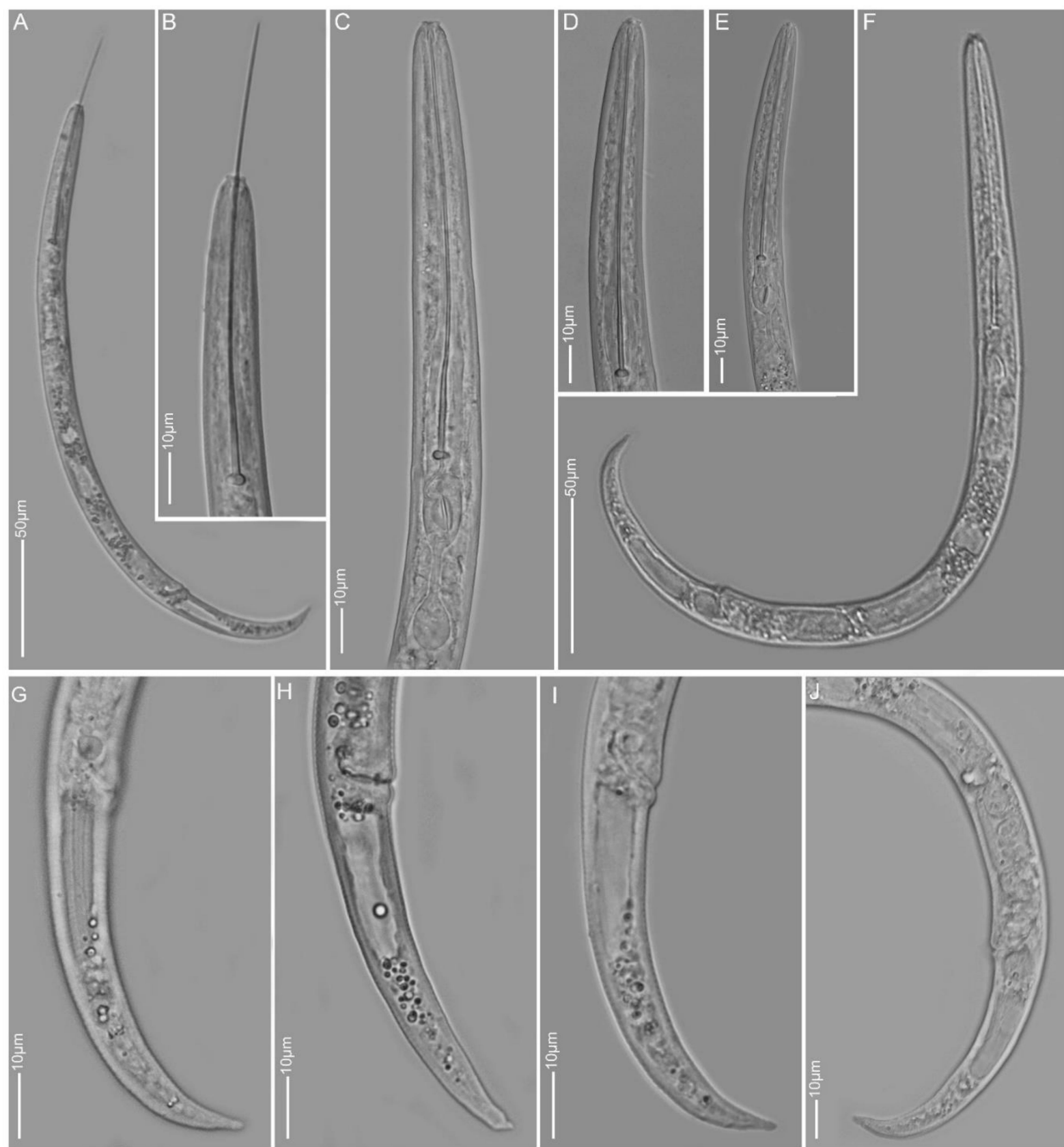

Figure 6. Light microscopy images of Paratylenchus idalimus females from sample BE20: (A,F) total body; (B-E) anterior region; (G-J) tail region.

\subsubsection{Paratylenchus microdorus}

Females (Sample BE9; Figure 7, Table 3): Body small, heat relaxed specimens open C- to 6-shape. Lateral field with four lateral lines. Deirids not observed. Cephalic region conical-truncate, submedian lobes sometimes slightly protruding. En face showing four submedian lobes and slit-like lateral amphidial openings. Stylet 11-15 $\mu \mathrm{m}$ long, cone $45-60 \%$ of stylet length. Pharynx about one-fifth of body length. Secretory-excretory pore between mid-isthmus and end bulb level. Spermatheca rounded, empty or filled with sperm cells. Vulval flaps prominent. Vulva located at $79-83 \%$ of body length from anterior end. Vagina oblique, reaching to half of the body width. Post-vulval uterine sac not seen. Tail 28-36 $\mu \mathrm{m}$ long, conoid and terminus pointed to subacute to sometimes finely rounded. 

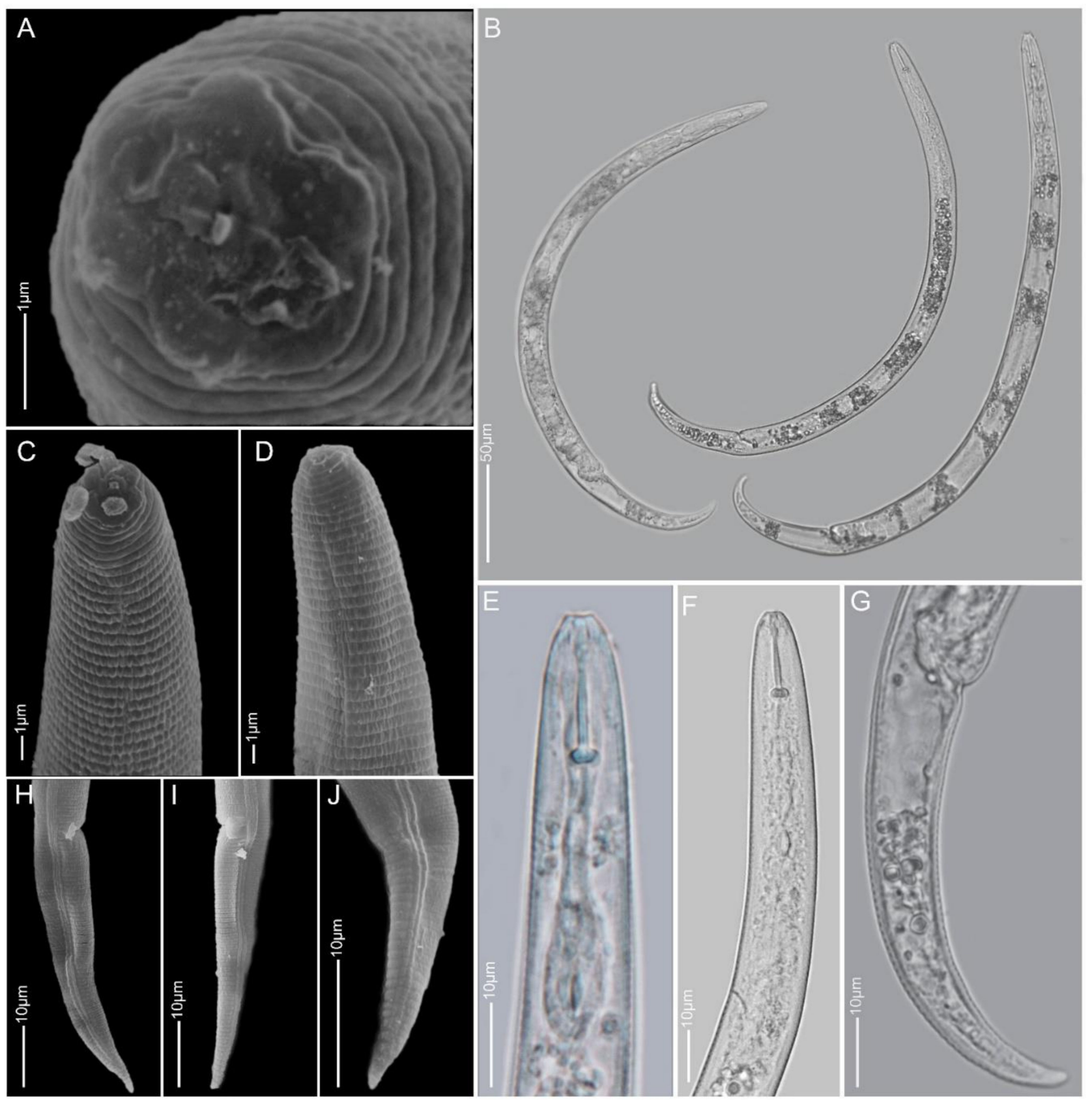

Figure 7. Light and scanning electron microscopy images of Paratylenchus microdorus females: (A) face view; (B) total body; (C-F) anterior region; (G-J) tail region.

Molecular characterisation: Three D2-D3 of 28S, three ITS, four 18S rRNA and three COI gene sequences were generated without intraspecific variability; the ITS and the COI sequences are new for this species. Only $300 \mathrm{bp}$ of the D2-D3 sequences were found to be homologous with four P. microdorus sequences from Germany (MF325254-MF325257; 98\% similarity; 5 bp difference). The $18 \mathrm{~S}$ rRNA sequences are $98-99 \%$ similar with P. microdorus from the Netherlands (AY284632 and AY284633; 8-15 out of 880 bp difference).

Remarks: Males were not found. Female morphologies and morphometrics agree well with the original description [59] and other populations [16,31], except for a slightly shorter stylet length (11-15 $\mu \mathrm{m}$ vs. 13-18 $\mu \mathrm{m})$. Wide variations in the tail termini have been reported for this species [31]. However, for the BE9 population, finely rounded to subacute female tail termini were commonly observed. This species is comparable to Paratylenchus recisus Siddiqi, 1996, Paratylenchus variabilis Raski, 1975 and P. veruculatus, with 
a female stylet length within 11-17 $\mu \mathrm{m}$, four lateral lines, presence of vulval flaps, secretoryexcretory pore at the posterior part of pharynx and vulva located at $78-87 \%$ of body length However, they differ from each another in having conical-truncate heads with sometimes slightly protruded submedian lobes in P. microdorus, broadly rounded to truncated head with central swallow depression in P. recisus, rounded to almost hemispherical head in $P$. variabilis and low and broadly rounded head in P. veruculatus. Only P. microdorus and $P$. veruculatus males have been reported to have weak stylets, while stylets in the males of the other two species are degenerated.

\subsubsection{Paratylenchus nanus}

Females (Sample BE11; Figure 8, Table 4): Heat relaxed specimens open C- to J-shape. Lateral field with four lateral lines. Deirids not observed. Cephalic region conical-rounded, in some specimens with sloping sides to rounded end, submedian lobes not protruding under LM. En face square shaped, revealing four submedian lobes, four distinct ridges around oral opening, lateral ridges slightly larger than dorso-ventral ridges, and two slit-like lateral amphidial openings. Stylet $27-31 \mu \mathrm{m}$ long, cone $67-78 \%$ of stylet length and knobs 3-5 $\mu \mathrm{m}$ across. Pharynx well developed, about one-fourth of body length. Hemizonid just above secretory-excretory pore, about two body annuli long. Secretoryexcretory pore between isthmus and end bulb level. Spermatheca rounded and filled with sperm cells. Vulval flaps present. Vulva located at $82-86 \%$ of body length from anterior end. Vagina oblique, reaching up to half of body width. Tail 19-26 $\mu \mathrm{m}$ long, conoid, often more pronounced curvature on dorsal side ending with sub-acute to finely rounded terminus.

Molecular characterisation: Seven D2-D3 of 28S, four ITS, four 18S rRNA and seven COI gene sequences were generated without any intraspecific sequence variations among four P. nanus populations-AR3, BE1, BE11 and BE18. The D2-D3 and the ITS sequences were, respectively, identical to KF242194, KF242197 and KF242267, KF242268 of P. nanus from Van den Berg et al. [22]

Remarks: Only in the BE11 population was a sufficient number of females recovered to allow morphological and morphometrical data comparisons, which agreed well with the original description [60] and subsequent descriptions of P. nanus $[19,22,58]$. Van den Berg et al. [22] reported two sibling species of P. nanus with different genotypestype A and type B (the latter of which was recently transferred to P. projectus) [61]. This correction suggests that the available 28S (MN720102-MN720103) and COI (MN734387 and MN734388) sequences of $P$. nanus from South Korea [48] were misidentified as they were found to be identical to the P. projectus sequences. Paratylenchus nanus is very similar to P. projectus and P. neoamblycephalus. It differs from P. projectus in having a conical-rounded vs. more trapezoid head shape and sperm-filled vs. empty spermathecae. It is differentiated from P. neoamblycephalus by more rounded vs. oval spermathecae and a conoid tailwith pronounced curvature on the dorsal side ending with a subacute or finely rounded terminus vs. a conoid tail with subacute terminus or almost acute tip. Furthermore, in our study we also observed that the ridges around the oral opening of the freshly killed specimens protruded more in $P$. neoamblycephalus compared to $P$. nanus when observed under LM. 

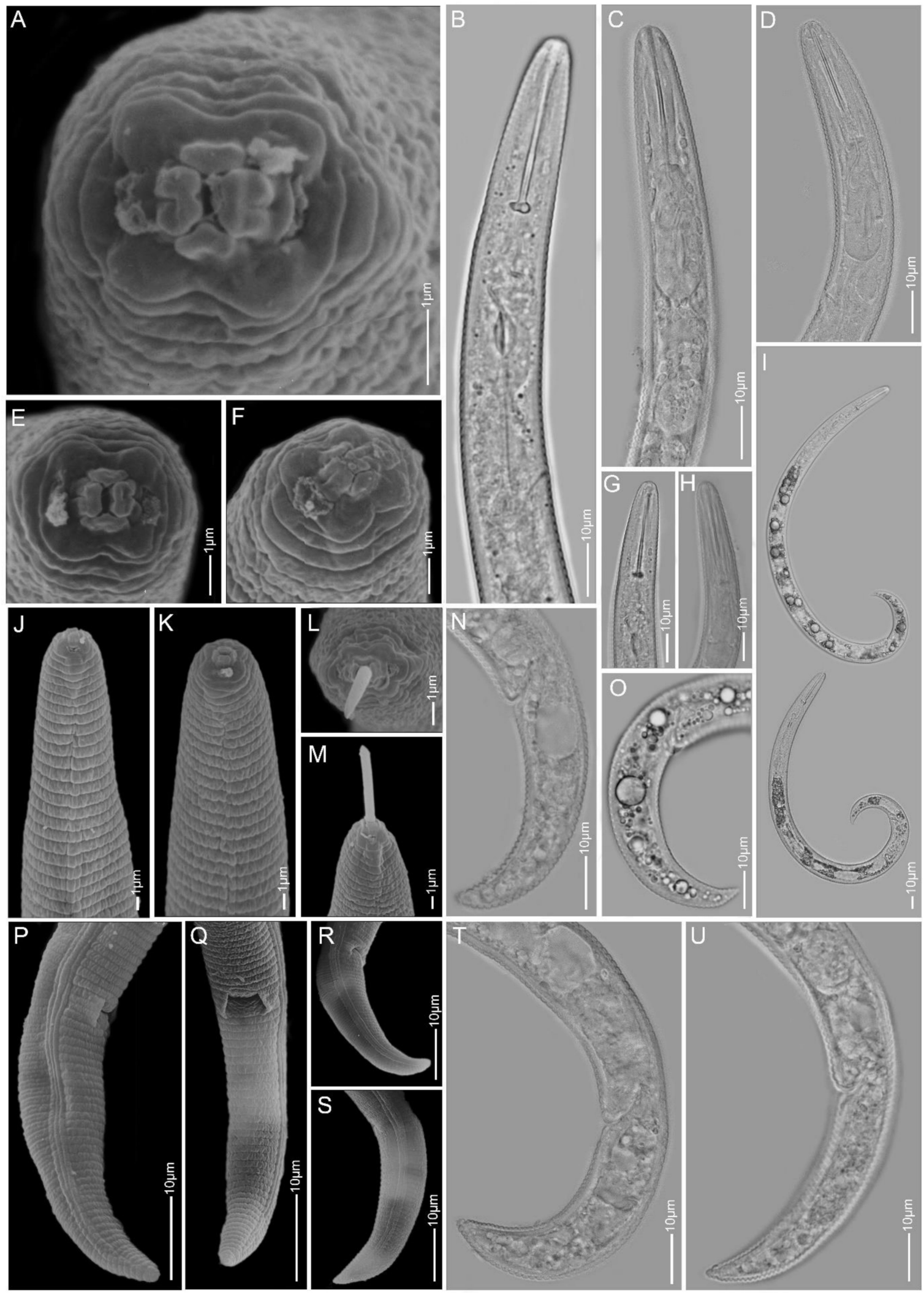

Figure 8. Light and scanning electron microscopy images of Paratylenchus nanus females: (A,E,F,L) face view; (B-D,G,H,J,K,M) anterior region; (I) total body; (N,O,P-U) tail region. 
Table 4. Female morphometrics of Paratylenchus nanus, Paratylenchus neoamblycephalus, Paratylenchus sp.2, Paratylenchus sp.D and Paratylenchus sp.F from fixed specimens mounted in glycerine. All measurements except for ratios and percentages are given in $\mu \mathrm{m}$ and in the form mean \pm stdev (range).

\begin{tabular}{|c|c|c|c|c|c|}
\hline Population & $\begin{array}{l}\text { P. nanus } \\
\text { (BE11) }\end{array}$ & $\begin{array}{l}\text { P. neoamblycephalus } \\
\text { (BE10) }\end{array}$ & $\begin{array}{l}\text { Paratylenchus } \\
\text { sp.2 (BE15) }\end{array}$ & $\begin{array}{l}\text { Paratylenchus } \\
\text { sp.D (BE20) }\end{array}$ & $\begin{array}{l}\text { Paratylenchus } \\
\text { sp.F (BE22) }\end{array}$ \\
\hline $\mathrm{n}$ & 30 & 15 & 16 & 11 & 17 \\
\hline $\mathrm{L}$ & $\begin{array}{l}318 \pm 15.8 \\
(287-352)\end{array}$ & $\begin{array}{l}337 \pm 20.2 \\
(301-367)\end{array}$ & $\begin{array}{l}347 \pm 20.7 \\
(308-389)\end{array}$ & $\begin{array}{l}328 \pm 36.1 \\
(285-387)\end{array}$ & $\begin{array}{l}300 \pm 21.1 \\
(264-339)\end{array}$ \\
\hline a & $\begin{array}{l}18.1 \pm 1.3 \\
(15.6-20.4)\end{array}$ & $\begin{array}{l}18.4 \pm 1.0 \\
(16.8-19.9)\end{array}$ & $\begin{array}{l}23.2 \pm 1.8 \\
(20.1-28.7)\end{array}$ & $\begin{array}{l}21.5 \pm 1.6 \\
(19.1-24.4)\end{array}$ & $\begin{array}{l}20.3 \pm 1.3 \\
(18.4-23.0)\end{array}$ \\
\hline$b$ & $3.9 \pm 0.3(3.5-4.8)$ & $4.3 \pm 0.5(3.4-4.9)$ & $3.9 \pm 0.3(3.5-4.4)$ & $3.6 \pm 0.3(3.3-4.1)$ & $4.0 \pm 0.3(3.7-4.6)$ \\
\hline c & $\begin{array}{l}14.8 \pm 1.3 \\
(12.8-16.8)\end{array}$ & $\begin{array}{l}14.6 \pm 1.5 \\
(12.9-16.6)\end{array}$ & $\begin{array}{l}13.2 \pm 0.7 \\
(12.3-14.4)\end{array}$ & $\begin{array}{l}14.5 \pm 1.4 \\
(12.0-15.8)\end{array}$ & $\begin{array}{l}12.9 \pm 0.8 \\
(11.8-14.0)\end{array}$ \\
\hline$c^{\prime}$ & $2.0 \pm 0.2(1.7-2.6)$ & $2.2 \pm 0.2(1.9-2.4)$ & $3.1 \pm 0.2(2.9-3.5)$ & $2.7 \pm 0.3(2.5-3.2)$ & $2.6 \pm 0.2(2.3-2.9)$ \\
\hline Max. body width & $\begin{array}{l}17.6 \pm 1.2 \\
(15.4-20.5)\end{array}$ & $\begin{array}{l}18.3 \pm 1.4 \\
(16.8-20.7)\end{array}$ & $\begin{array}{l}15.1 \pm 1.3 \\
(13.2-16.8)\end{array}$ & $\begin{array}{l}15.3 \pm 2.2 \\
(13.2-19.9)\end{array}$ & $\begin{array}{l}14.8 \pm 1.1 \\
(13.3-16.6)\end{array}$ \\
\hline Stylet length & $\begin{array}{l}28.8 \pm 1.2 \\
(26.7-31.2)\end{array}$ & $\begin{array}{l}33.4 \pm 0.9 \\
(32.0-34.3)\end{array}$ & $\begin{array}{l}28.4 \pm 1.5 \\
(26.5-31.4)\end{array}$ & $\begin{array}{l}27.5 \pm 1.0 \\
(25.7-28.9)\end{array}$ & $\begin{array}{l}27.6 \pm 1.2 \\
(25.3-29.6)\end{array}$ \\
\hline Cone length & $\begin{array}{l}20.2 \pm 1.4 \\
(17.7-23.4)\end{array}$ & $\begin{array}{l}22.6 \pm 0.9 \\
(21.4-24.5)\end{array}$ & $\begin{array}{l}19.2 \pm 1.0 \\
(17.5-20.8)\end{array}$ & $\begin{array}{l}17.7 \pm 0.8 \\
(17.0-19.2)\end{array}$ & $\begin{array}{l}18.5 \pm 0.9 \\
(17.1-20.3)\end{array}$ \\
\hline Cone\%stylet & $\begin{array}{l}70.1 \pm 3.6 \\
(64.9-78.5)\end{array}$ & $\begin{array}{l}67.8 \pm 2.8 \\
(63.0-72.5)\end{array}$ & $\begin{array}{l}67.5 \pm 2.0 \\
(64.4-71.2)\end{array}$ & $\begin{array}{l}64.3 \pm 2.0 \\
(60.7-67.3)\end{array}$ & $\begin{array}{l}67.1 \pm 1.3 \\
(65.2-69.5)\end{array}$ \\
\hline Knob width & $3.9 \pm 0.4(3.1-4.6)$ & $4.8 \pm 0.2(4.4-5.1)$ & $4.1 \pm 0.3(3.5-4.7)$ & $4.0 \pm 0.3(3.5-4.6)$ & $3.6 \pm 0.3(3.3-4.1)$ \\
\hline Pharynx length & $\begin{array}{c}81.6 \pm 5.4 \\
(65.4-91.1)\end{array}$ & $\begin{array}{l}79.7 \pm 9.1 \\
(65.7-93.8)\end{array}$ & $\begin{array}{l}88.2 \pm 4.1 \\
(78.0-96.7)\end{array}$ & $\begin{array}{l}89.6 \pm 7.3 \\
(76.0-104)\end{array}$ & $\begin{array}{c}74.8 \pm 4.9 \\
(67.7-83.1)\end{array}$ \\
\hline $\begin{array}{l}\text { Ant. end to SE } \\
\text { pore }\end{array}$ & $\begin{array}{l}65.7 \pm 6.0 \\
(54.7-75.0)\end{array}$ & $\begin{array}{l}63.9 \pm 4.7 \\
(52.2-70.0)\end{array}$ & $\begin{array}{l}72.3 \pm 4.5 \\
(64.2-90.0)\end{array}$ & $\begin{array}{l}74.6 \pm 6.8 \\
(66.7-90.2)\end{array}$ & $\begin{array}{l}63.0 \pm 5.4 \\
(51.5-70.6)\end{array}$ \\
\hline SE pore\%L & $\begin{array}{l}20.6 \pm 1.4 \\
(17.0-22.5)\end{array}$ & $\begin{array}{l}19.0 \pm 1.5 \\
(15.8-21.7)\end{array}$ & $\begin{array}{l}20.8 \pm 1.3 \\
(19.4-23.5)\end{array}$ & $\begin{array}{l}23.6 \pm 0.9 \\
(22.1-24.8)\end{array}$ & $\begin{array}{l}21.0 \pm 1.6 \\
(18.6-24.2)\end{array}$ \\
\hline Ant. end to vulva & $\begin{array}{l}270 \pm 18.8 \\
(249-330)\end{array}$ & $\begin{array}{l}276 \pm 15.0 \\
(247-296)\end{array}$ & $\begin{array}{l}286 \pm 17.1 \\
(252-313)\end{array}$ & $\begin{array}{l}270 \pm 27.9 \\
(239-320)\end{array}$ & $\begin{array}{l}217 \pm 17.2 \\
(217-278)\end{array}$ \\
\hline $\mathrm{V} \%$ & $\begin{array}{l}83.8 \pm 1.1 \\
(81.7-85.7)\end{array}$ & $\begin{array}{l}81.9 \pm 0.9 \\
(80.7-83.8)\end{array}$ & $\begin{array}{l}82.2 \pm 0.8 \\
(81.2-83.5)\end{array}$ & $\begin{array}{l}83.7 \pm 1.0 \\
(81.8-85.2)\end{array}$ & $82 \pm 0.7(80.9-83.5)$ \\
\hline $\begin{array}{l}\text { Body width at } \\
\text { anus }\end{array}$ & $\begin{array}{l}10.9 \pm 0.9 \\
(9.3-12.6)\end{array}$ & $\begin{array}{l}10.6 \pm 0.8 \\
(9.4-12.0)\end{array}$ & $8.6 \pm 0.7(7.6-9.8)$ & $8.0 \pm 0.6(7.3-8.7)$ & $9.0 \pm 0.6(8.0-9.9)$ \\
\hline Tail length & $\begin{array}{l}21.7 \pm 1.9 \\
(18.9-25.5)\end{array}$ & $\begin{array}{l}23.2 \pm 2.6 \\
(20.2-27.8)\end{array}$ & $\begin{array}{l}26.1 \pm 2.0 \\
(23.0-28.7)\end{array}$ & $\begin{array}{l}22.0 \pm 3.0 \\
(18.0-25.6)\end{array}$ & $\begin{array}{l}23.1 \pm 2.4 \\
(20.0-26.5)\end{array}$ \\
\hline
\end{tabular}

\subsubsection{Paratylenchus neoamblycephalus}

Females (Sample BE10; Figure 9, Table 4): Heat relaxed specimens open C-shape. Lateral field with four lateral lines. Deirids clearly visible on SEM images. Cephalic region truncated-rounded, submedian lobes sometimes very slightly protruding under LM. En face showing four rounded to oval submedian lobes, four ridges around oral opening, lateral ridges thicker than dorso-ventral ridges and seen as a protruding bi-lobed structure under LM. Stylet 32-34 $\mu \mathrm{m}$ long, cone $63-73 \%$ of stylet length, knobs $4-5 \mu \mathrm{m}$ across. Pharynx about one-fourth of body length. Secretory-excretory pore between mid-isthmus and end bulb level, with swellings sometimes seen at the duct near the opening in freshly killed specimens. Spermatheca oval and filled with sperm cells. Vulval flaps present. Vulva located at $81-84 \%$ of body length from anterior end. Vagina oblique and reaching up to half of body width. Tail 20-28 $\mu \mathrm{m}$ long, conoid and terminating to sub-acute to almost acute tip. 

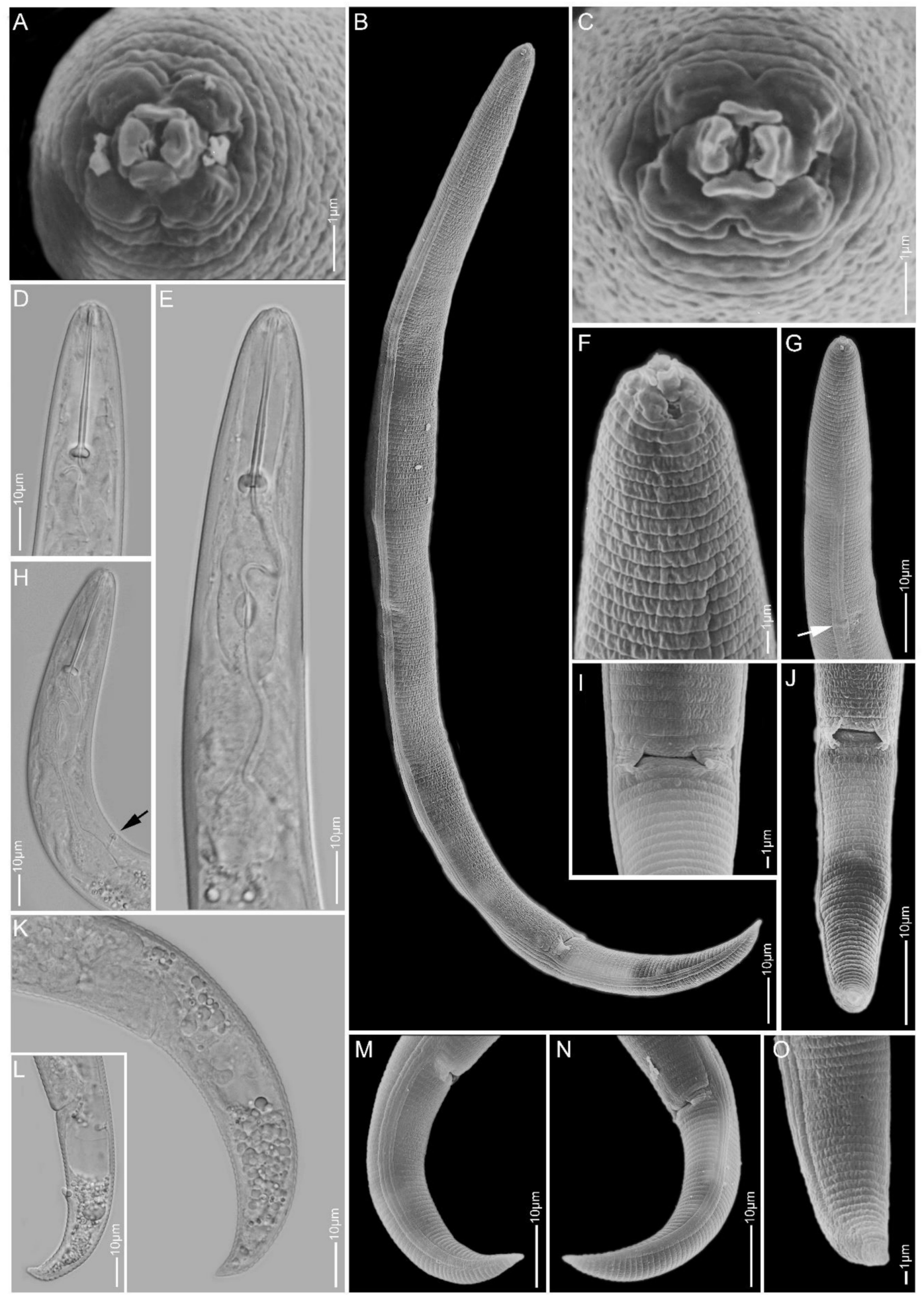

Figure 9. Light and scanning electron microscopy images of Paratylenchus neoamblycephalus females: (A,C) face view; (B) total body; (D-H) anterior region; (I-O) tail region; arrows pointed to secretory-excretory pore in (H) and deirid in (G).

Males: Two males were obtained with very thin stylets in freshly killed specimens, which were not visible after fixation, and spicules of $24 \mu \mathrm{m}$ long. Their conspecificity with the females was confirmed by identical COI and D2-D3 sequences.

Molecular characterisation: Four D2-D3 of 28S, five ITS, six 18S rRNA and eight COI gene sequences were generated without intraspecific sequence variations. The $18 \mathrm{~S}$ and the COI sequences are new for this species. The D2-D3 sequences were found to be identical to 
KF242189 and KF242190 of an unidentified Paratylenchus sp.6 from the USA [22], which is considered here as P. neoamblycephalus. However, the D2-D3 sequences were only $89 \%$ similar (79 out of $710 \mathrm{bp}$ difference) with MG925221 and 92\% similar (43 out of $546 \mathrm{bp}$ difference) with MK506807 of P. neoamblycephalus from the USA and Iran, and named here as type A and type B, respectively. Interestingly, we observed 17 ambiguous nucleotide sites in the American P. neoamblycephalus type B sequence, which was found to be similar to $P$. projectus (previously P. nanus type B; KF242198-KF242201; 98\% similarity; 16-20 out of $690 \mathrm{bp}$ difference) after Van den Berg et al. [22,61]. On the other hand, the Iranian P. neoamblycephalus sequence [24] was similar to P. nanus (KF242194 and KF242197; 95\% similarity; 27 out of 575 bp difference) [22]. Furthermore, our ITS sequences were only $74 \%$ similar (222 out of $865 \mathrm{bp}$ difference) to MK506794 of P. neoamblycephalus type A generated from the same Iranian population.

Remarks: This species is reported for the first time in Belgium. Female morphology and morphometrics agree well with the original description from Germany [18] and to subsequent descriptions from Poland [19]. Paratylenchus neoamblycephalus is very similar to P. nanus and a comparison is provided above.

\subsubsection{Paratylenchus straeleni}

Females (Sample BE15; Figure 10, Table 2): Heat relaxed specimens J- to C-shape. Lateral field with four lateral lines. Deirids clearly visible under SEM. Cephalic region conical-rounded to sometimes slightly truncated, submedian lobes not protruded. Stylet straight to slightly curved, 54-59 $\mu \mathrm{m}$ long, cone $76-83 \%$ of stylet length, knobs 3-5 $\mu \mathrm{m}$ across. Pharynx roughly one-fourth of body length. Secretory-excretory pore between isthmus and end bulb level. Spermatheca rounded to sometimes slightly ovoid and filled with sperm cells. Vulval flaps distinct. Vulva located at $80-84 \%$ of body length from anterior end. Vagina oblique, occasionally reaching to two-third of body width. Tail conical, 31-41 $\mu \mathrm{m}$ long, and terminus sharply pointed to minutely rounded.

Males: Two males were recovered without stylets and with spicule lengths of 20 and $22 \mu \mathrm{m}$, respectively. Their conspecificity with the females was confirmed by identical COI sequences.

Molecular characterisation: One D2-D3 of 28S, two ITS (99\% similarity; 4 out of $830 \mathrm{bp}$ difference), three identical $18 \mathrm{~S}$ rRNA and five identical COI gene sequences were generated from the BE15 population. From another population (BE11), single D2-D3, ITS and 18S sequences and three identical COI sequences were also generated. All the sequences from both populations showed no intraspecific variation, except for the ITS sequences. The 18 S sequences were $99 \%$ similar (3-5 out of 930 bp difference) with $P$. straeleni from the Netherlands (AY284630 and AY284631). The D2-D3 sequences were 97-99\% similar (11-18 out of $700 \mathrm{bp}$ difference) with four $P$. straeleni sequences-i.e., MK506804 from Iran [24], KM875547 from Turkey [42], and KF242235 and KF242236 from the USA [22]. The COI sequences were generated for the first time for this species. Remarkably, the Belgian ITS sequences were only $62 \%$ similar ( $295 \mathrm{bp}$ difference) to the Iranian P. straeleni sequence (MK506791) of Hesar et al. [24].

Remarks: Female morphology and morphometrics agree well with the original description, also from Belgium [62], and subsequent descriptions of globally distributed P. straeleni populations [31]. This species is comparable to $P$. goodeyi as described above. 


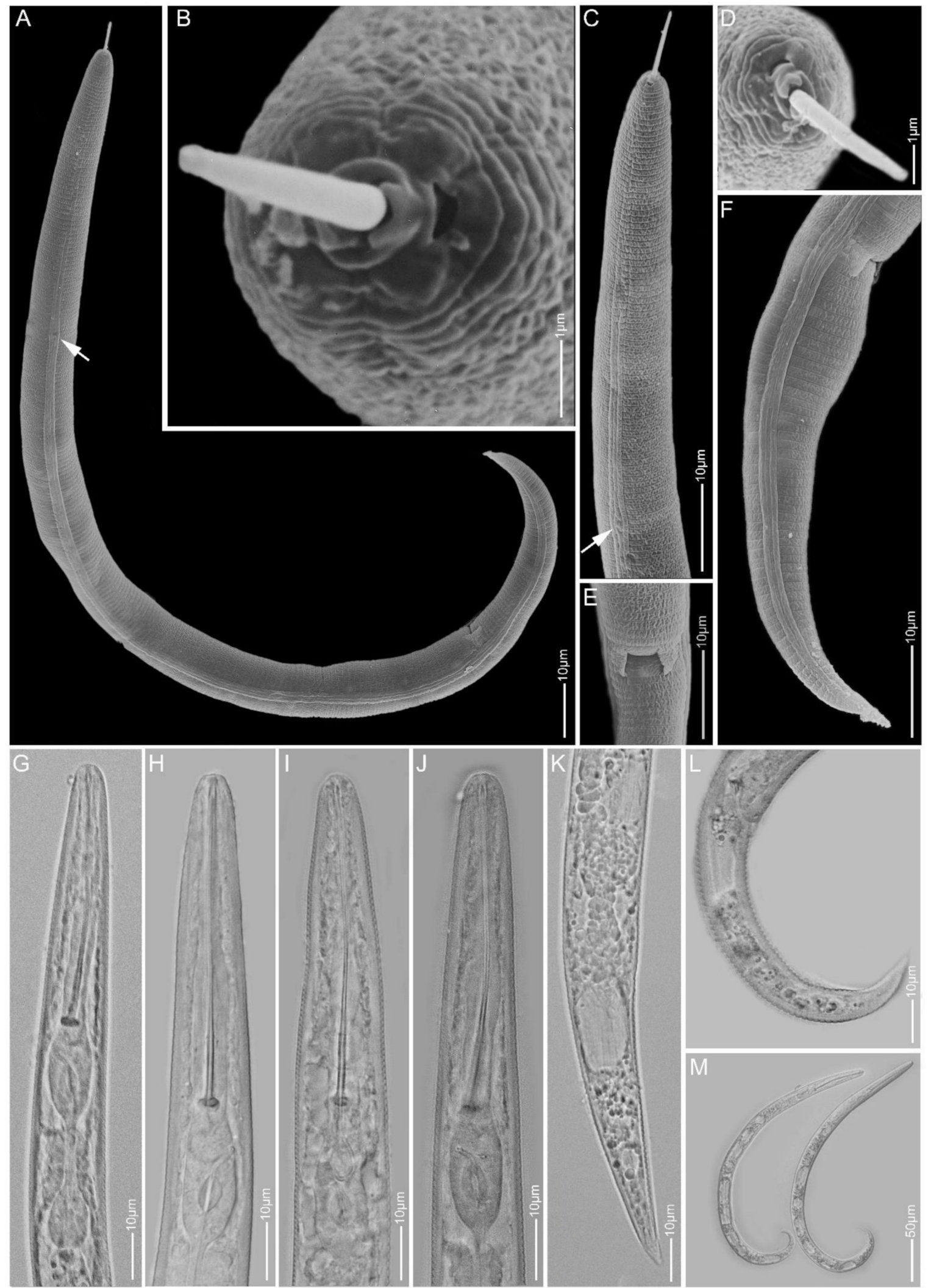

Figure 10. Light and scanning electron microscopy images of Paratylenchus straeleni females: (A) whole body; (B,D) face view; (C,G-J) anterior region; (E) vulva region; $(\mathbf{F}, \mathbf{K}, \mathbf{L})$ tail region; $(\mathbf{M})$ total body; arrows pointed to deirids in $(\mathbf{A}, \mathbf{C})$. 


\subsubsection{Paratylenchus veruculatus}

Females (Sample BE20; Figure 11, Table 3): Heat relaxed specimens open C-shape to slightly ventrally curved. Lateral field with four lateral lines. Deirids not observed. Cephalic region broadly rounded, submedian lobes not protruding. En face rectangular with indistinct submedian lobes, four irregular ridges around oral opening and lateral amphidial openings. Stylet $13-15 \mu \mathrm{m}$ long, cone $60-65 \%$ of stylet length, knobs about $3 \mu \mathrm{m}$ across. Pharynx roughly one-fourth of total body length. Hemizonid two body annuli long, usually visible just above secretory-excretory pore. Secretory-excretory pore between mid-isthmus and end bulb level. Spermatheca rounded and filled with sperm cells, young females with empty spermatheca also seen. Vulval flaps prominent. Vulva located at $84-90 \%$ of body length from anterior end. Vagina oblique and long, reaching up to three-fourth of body width. Tail 14-19 $\mu \mathrm{m}$ long, conoid with often broadly rounded to sometimes finely rounded terminus.
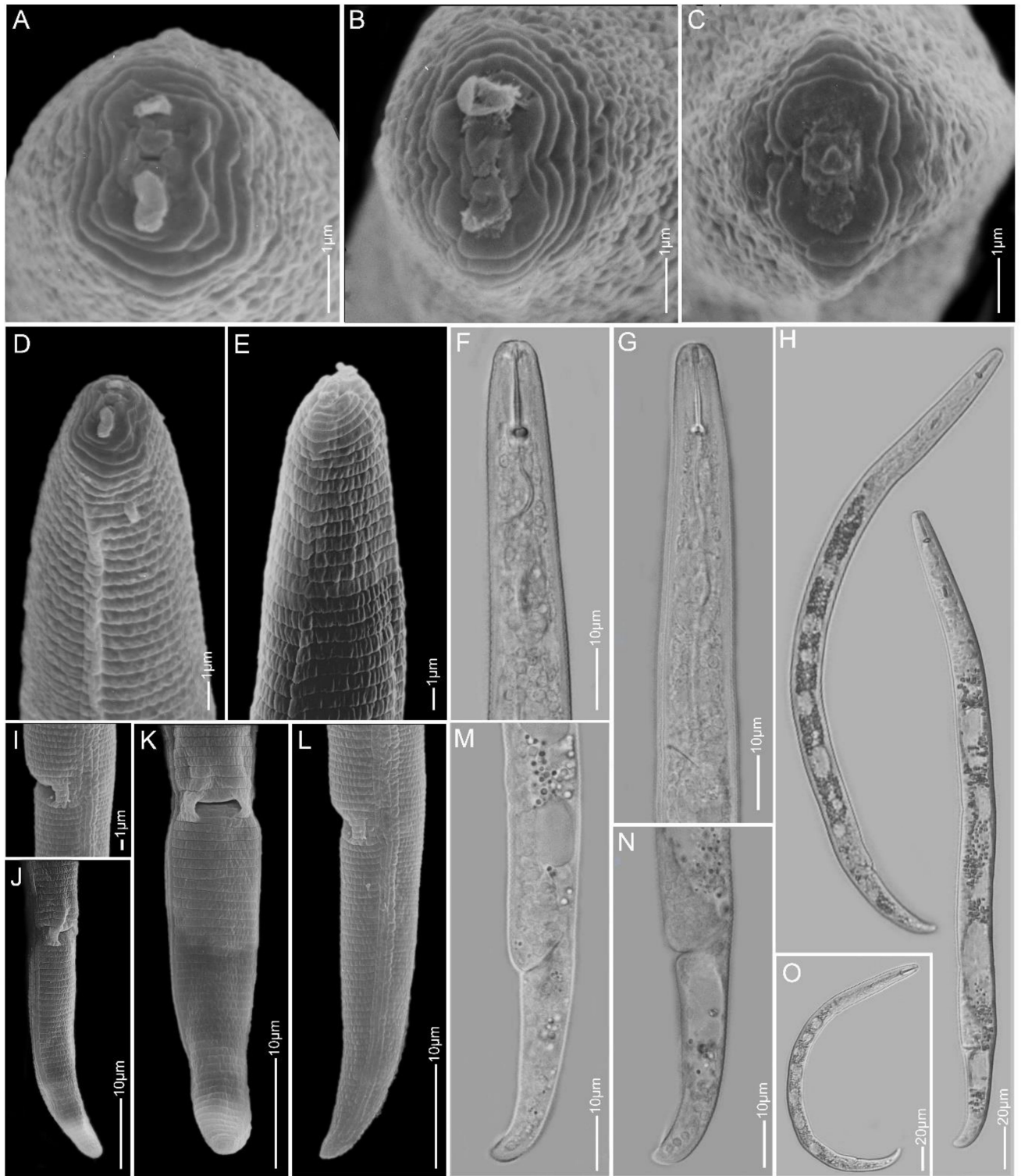

Figure 11. Light and scanning electron microscopy images of Paratylenchus veruculatus females: (A-C) face view; (D-G) anterior region; $(\mathbf{H}, \mathbf{O})$ total body; $(\mathbf{I}-\mathbf{N})$ lateral field and tail region. 
Molecular characterisation: Five D2-D3 of 28S (99\% similarity; 1-2 out of 720 bp difference) and two identical 18S rRNA and seven COI gene (97-100\% similarity; $12-13$ out of $410 \mathrm{bp}$ difference) sequences were generated for the first time for this species.

Remarks: Female morphology and morphometrics agree well with the original description [63] and with other populations [19,31]. This species is comparable to other species with a short stylet such as P. microdorus, $P$. recisus and P. variabilis (see also above).

\subsubsection{Paratylenchus sp.2}

Females (Sample BE15; Figure 12, Table 4): Heat relaxed specimens open C-shape. Lateral field with four lateral lines. Deirids observed under SEM. Cephalic region conicalrounded, sometimes slightly trapezoid, submedian lobes not protruding under LM. En face square-shaped, showing four rounded, poorly separated submedian lobes, four ridges around oral opening, lateral ridges more prominent and larger than dorso-ventral ridges. Stylet $27-31 \mu \mathrm{m}$ long, cone $64-71 \%$ of stylet length, knobs about $4 \mu \mathrm{m}$ across. Pharynx well developed, about one-fourth of body length. Hemizonid just above secretory-excretory pore, about two body annuli long. Secretory-excretory pore between mid-isthmus and end bulb level. Spermatheca rounded to occasionally slightly ovoid, filled with sperm cells. Vulval flaps prominent. Vulva located at $81-84 \%$ of body length from anterior end. Vagina oblique, reaching up to two-third of body width. Tail 23-29 $\mu \mathrm{m}$ long, conoid, slender and terminating with finely rounded tip.

Molecular characterisation: Two identical sequences each of D2-D3 of 28S, ITS, 18S rRNA as well as the COI gene were generated. The D2-D3 and ITS sequences were found to be, respectively, identical to KF242220 and KF242221 and 99\% similar (five out of 750 bp difference) to KF242243 of Paratylenchus sp.2, which was identified as a member of the P. hamatus species complex [22]. The $18 \mathrm{~S}$ and COI sequences were generated for the first time.

Remarks: Males were not found. The female morphology and morphometrics are in agreement with the description of P. hamatus [64]. Based on morphology and D2-D3 and ITS sequences, Van den Berg et al. [22] considered P. hamatus as a species complex containing several species, including $P$. hamatus sensu stricto collected from the type locality, and Paratylenchus sp.1 and Paratylenchus sp.2, collected from other places in California. Paratylenchus sp.1 is identified as representative of P. tenuicaudatus. Paratylenchus sp. 2 is morphologically similar with $P$. hamatus sensu stricto but differs based on D2-D3 and ITS sequences [22], and this species appears to be not only present in the USA (California) but also in Belgium and Kyrgyzstan. 

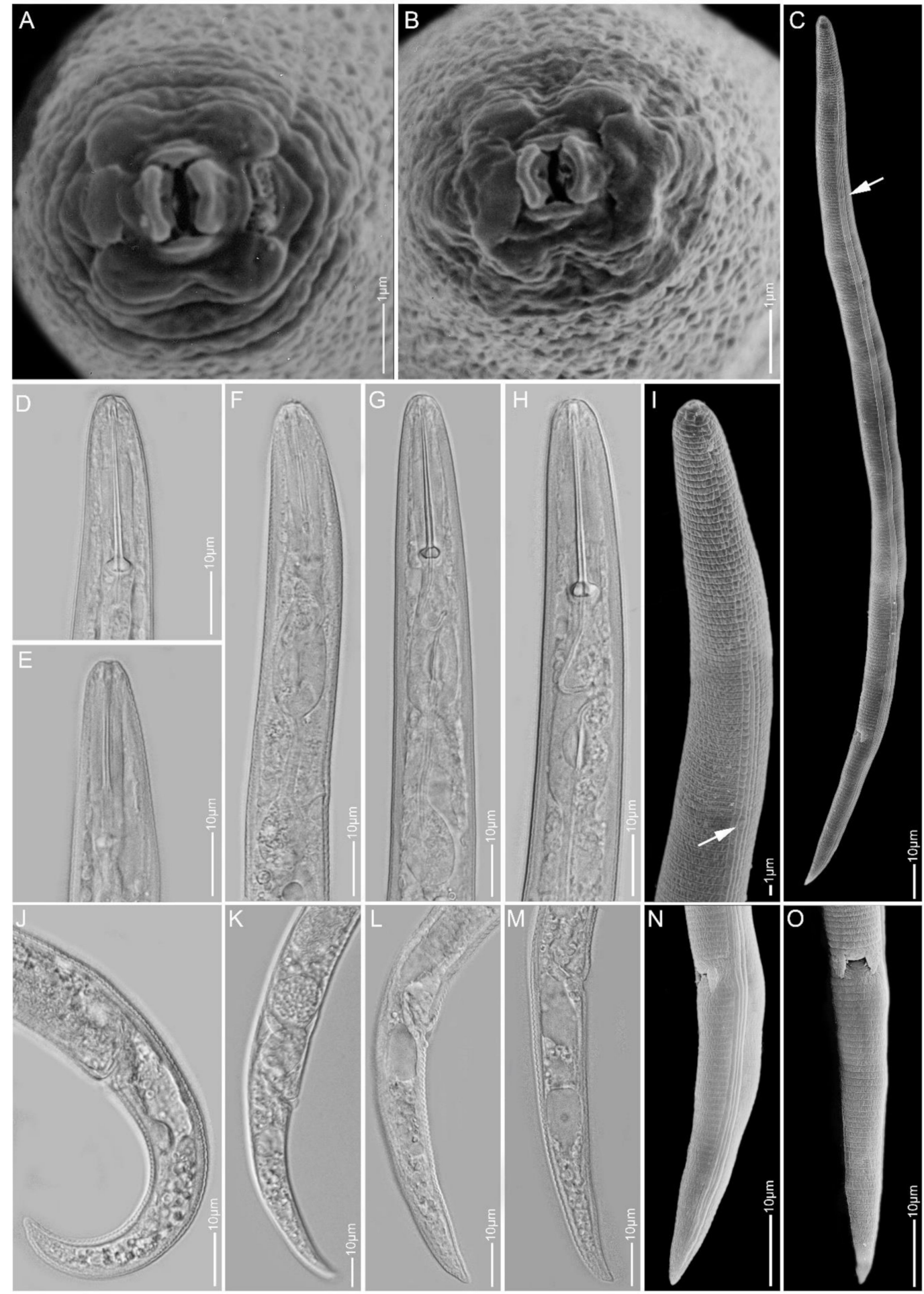

Figure 12. Light and scanning electron microscopy images of Paratylenchus sp.2 females: (A,B) face view; (C) total body; (D-I) anterior region; (J-O) tail region; arrows pointed at deirids in (C,I).

\subsubsection{Paratylenchus sp.BE11}

Females (Sample BE11; $n=3$; Figure 13): Body about $0.3 \mathrm{~mm}$ long with maximum body width of about $15 \mu \mathrm{m}$, heat relaxed specimens open C- to 6-shape. Lateral field with four lateral lines. Deirids not observed. Head broadly rounded, submedian lobes not protruded, cephalic sclerotization strong. Stylet about $15 \mu \mathrm{m}$ long, cone $60 \%$ of stylet length, knobs $3 \mu \mathrm{m}$ across. Pharynx about one-fourth of body length. Secretory-excretory pore at the 
level of pharyngeal end bulb or about $70 \mu \mathrm{m}$ from anterior end. Spermatheca rounded and filled with sperm cells. Vulval flaps small and rounded. Vulva located at $80-82 \%$ of body length from anterior end. Tail 25-32 $\mu \mathrm{m}$, conoid with bluntly rounded tip.

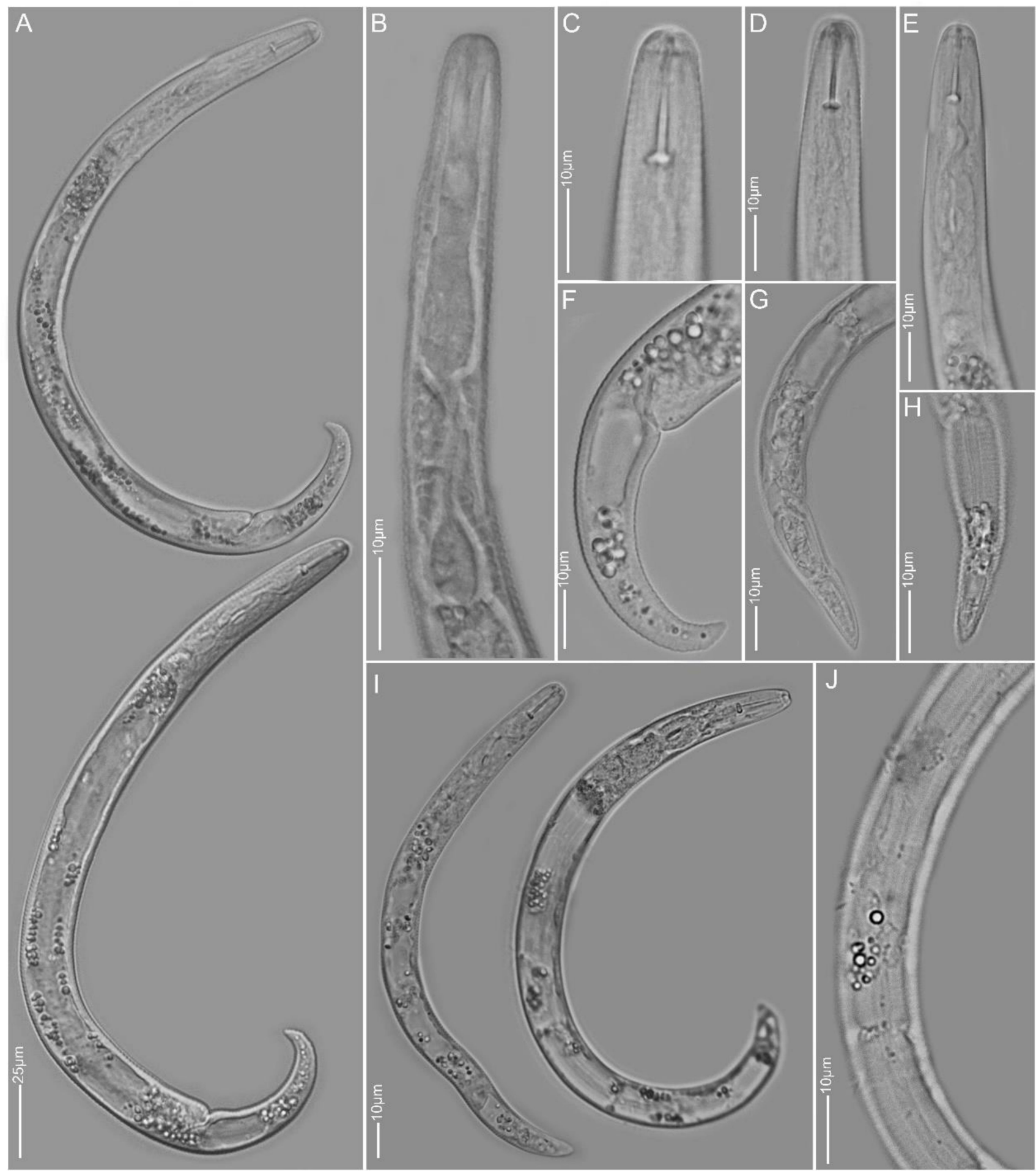

Figure 13. Light microscopy images of Paratylenchus sp.BE11 females: (A) total body; (B-E) anterior region; (F-H) tail region; (I) total body; (J) lateral field.

Molecular characterisation: Three identical D2-D3 of 28S, one ITS rRNA and two identical COI gene sequences were generated. 
Remarks: No males were found. Female description is based on only three freshly killed specimens, while sufficient specimens are needed for a comprehensive species characterisation. The female morphology is close to P. variabilis, P. veruculatus and Paratylenchus vexans Thorne and Malek, 1986. These four species have more or less broadly rounded heads with non-protruding submedian lobes, stylet lengths in the range of 12-18 $\mu \mathrm{m}$, four lateral lines, sperm-filled spermathecae, vulval flaps and conoid tails with more or less rounded termini. However, our population appears to have a stronger cephalic sclerotisation and slightly more anteriorly located vulvae ( $80-82 \%$ vs. $80-87 \%)$ compared to the other three species. This species is a sister to P. microdorus in the D2-D3 tree (96\% similarity; 27 out of $740 \mathrm{bp}$ difference), ITS tree (93\% similarity; 37 out of $530 \mathrm{bp}$ difference) as well as the COI tree (91\% similarity; 36 out of $420 \mathrm{bp}$ difference). It can, however, be readily morphologically distinguished from P. microdorus (see above).

\subsubsection{Paratylenchus sp.D}

Females (Sample BE20; Figure 14, Table 4): Heat relaxed specimens open C-shape. Lateral field with four lateral lines. Cephalic region conical-rounded to sometimes slightly trapezoid, submedian lobes not protruding under LM. Deirids visible under SEM. En face showing four well-separated rounded submedian lobes and four ridges around oral opening. Stylet $26-29 \mu \mathrm{m}$ long, cone $61-67 \%$ of stylet length, knobs about $4 \mu \mathrm{m}$ across. Pharynx about one-fourth of body length. Hemizonid just above secretory-excretory pore, about two body annuli long. Secretory-excretory pore between mid-isthmus and end bulb level. Spermatheca empty. Vulval flaps prominent, commonly rounded. Vulva located at $82-85 \%$ of body length from anterior end. Vagina oblique reaching up to half of body width. Tail 18-26 $\mu \mathrm{m}$ long, conoid with finely rounded to bluntly rounded terminus, sometimes dorsally sinuate.

Molecular characterisation: Seven D2-D3 of 28S (99\% similarity; one out of $730 \mathrm{bp}$ difference), four ITS, five $18 \mathrm{~S}$ rRNA and eleven COI gene sequences were generated without intraspecific sequence variation.

Remarks: Males were not found. The female morphology and morphometrics is close to P. projectus and Paratylenchus neoprojectus Wu and Hawn, 1975. The cephalic region of females were seen with both rounded to trapezoid shape, secretory-excretory pore located between mid-isthmus to end bulb level, empty spermatheca and tail termini which fit both the above two species. However, the molecular data appears to be different from any available sequences including that of $P$. projectus. A comparative study of this species with type specimens of $P$. neoprojectus and its molecular information should further confirm whether or not this species is $P$. neoprojectus. 


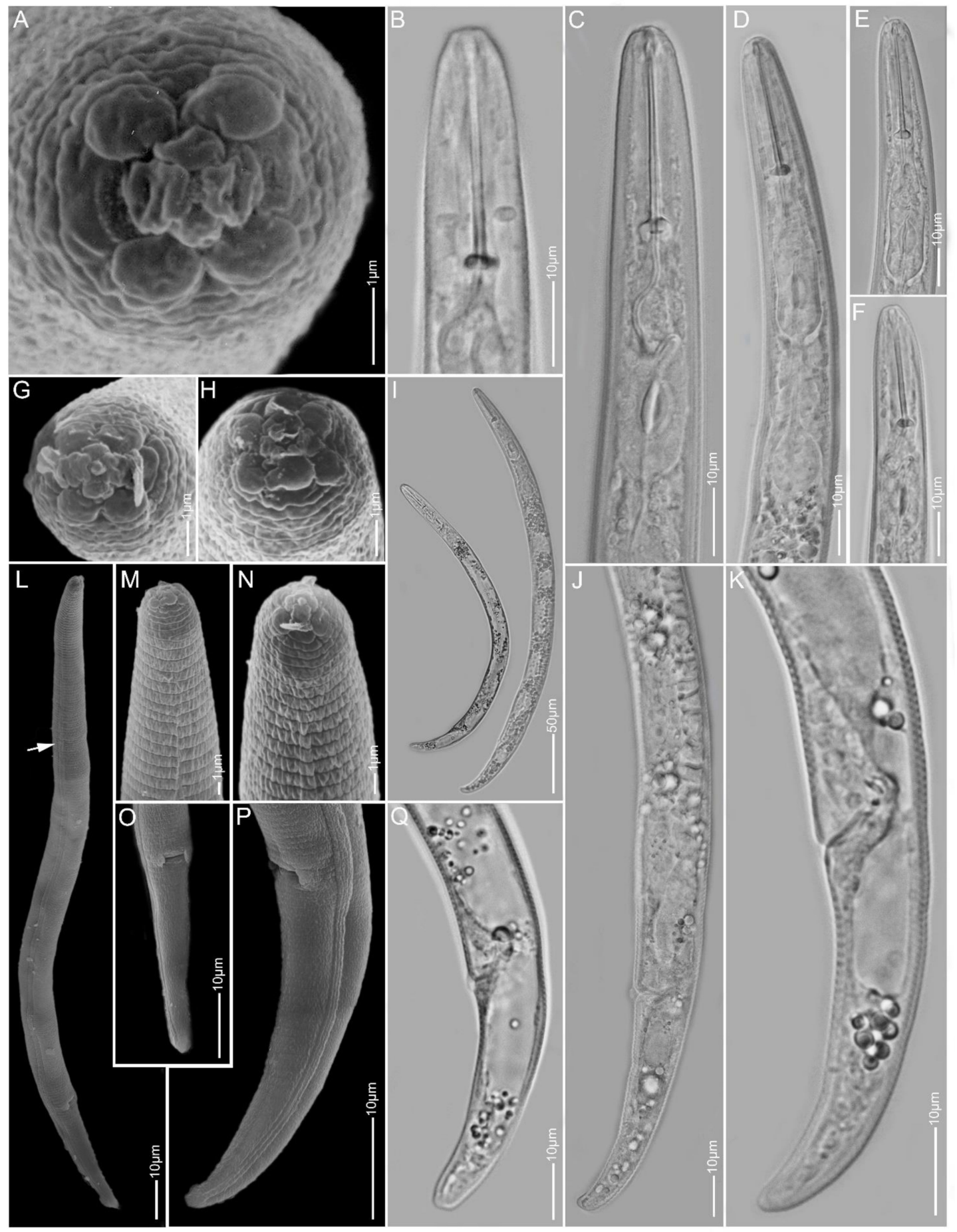

Figure 14. Light and scanning electron microscopy images of Paratylenchus sp.D females; (A,G,H) face view; (B-F,M,N) anterior region; $(\mathbf{I}, \mathbf{L})$ total body; $(\mathbf{J}, \mathbf{K}, \mathbf{O}-\mathbf{Q})$ tail region; arrow pointed to deirid in $\mathrm{L}$.

\subsubsection{Paratylenchus sp.F}

Females (Sample BE22; Figure 15, Table 4): Heat relaxed specimen open C- to 6-shape. Lateral field with four lateral lines. Deirids present. Cephalic region conical-truncate, slightly rounded in few specimens, submedian lobes not protruding under LM. En face square-shaped, showing four rounded submedian lobes, four ridges around oral opening, dorso-ventral ridges much larger than the lateral ridges, slit like amphidial apertures laterally. Stylet $25-30 \mu \mathrm{m}$ long, cone $65-70 \%$ of stylet length, knobs about $4 \mu \mathrm{m}$ across. Pharynx about one-fourth of body length. Hemizonid just above secretory-excretory pore, 
about two body annuli long. Secretory-excretory pore between mid-isthmus and end bulb level. Spermatheca oval to elongated and filled with sperm cells. Vulval flaps rounded to oval and very prominent. Vulva located at $81-84 \%$ of body length from anterior end. Vagina oblique, reaching up to two-third of body width. Tail 20-26 $\mu \mathrm{m}$ long, conoid with regularly bluntly rounded terminus.
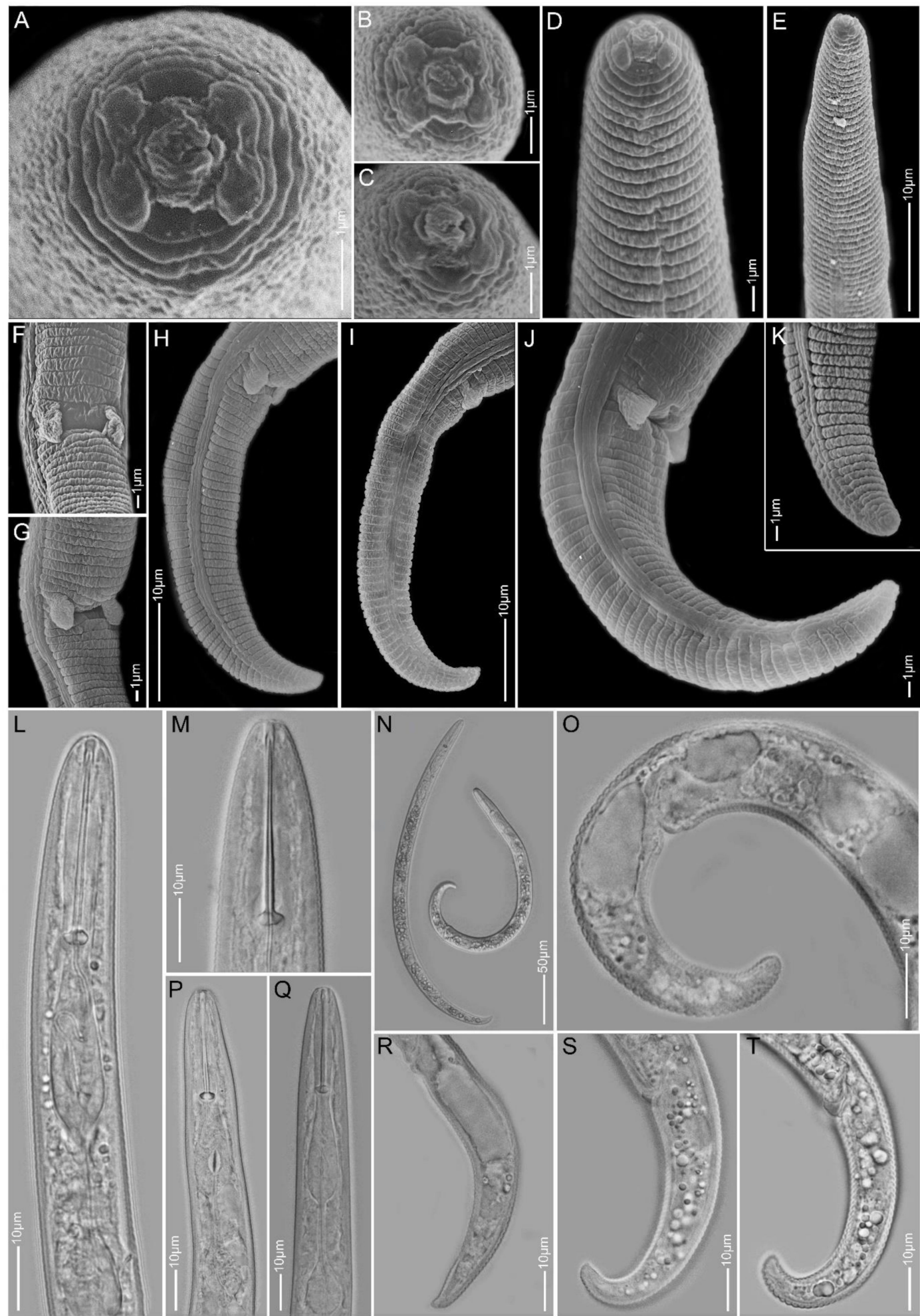

Figure 15. Light and scanning electron microscopy images of Paratylenchus sp.F females: (A-C) face view; (D,E,L,M,P,Q) anterior region; $(\mathbf{F}, \mathbf{G})$ vulva region; $(\mathbf{H}-\mathbf{K}, \mathbf{O}, \mathbf{R}-\mathbf{T})$ tail region; $(\mathbf{N})$ total body. 
Males: Heat relaxed specimen curved slightly ventrally, about the same body length as females but slightly slender. Cephalic region conoid and rounded. Stylet and pharynx degenerated. Secretory-excretory pore at about one-fifth of body length from anterior end. Spicule arcuate ventrally, about $21.5 \mu \mathrm{m}$ in length. Gubernaculum 3-5 $\mu \mathrm{m}$ long. Tail conical with finely rounded tip. Conspecificity of males with females was confirmed by identical D2-D3, 18S and ITS sequences.

Molecular characterisation: Five D2-D3 of 28S, three ITS, four $18 \mathrm{~S}$ rRNA and three COI gene sequences were generated without intraspecific sequence variations. The D2-D3, 18S and COI sequences were found to be identical, respectively, to MN783707, MN783708, MN783668-MN783670 and MN782407-MN782413 of Paratylenchus sp.F [47], while the ITS sequences were generated for the first time.

Remarks: Specimens belong to the same population as Paratylenchus sp.F in Etongwe et al.'s work [47]. Detailed morphological reanalysis revealed very close similarity to $P$. nanus. Nevertheless, the submedian lobes of this species appear to be somewhat more rounded than that of $P$. nanus based on SEM images and the vulval flaps also appear to be more pronounced and rounded compared to that of the latter. However, these characteristics need careful additional observations based on more specimens from both species. All four gene sequences of Paratylenchus sp.F were closest to the sequences of P. elachistus and phylogenetic analysis revealed their highly supported (PP $>90 \%)$ sister relationship. However, this species is morphologically different from P. elachistus en face, with rounded vs. poorly differentiated submedian lobes, stylet lengths of $25-30 \mu \mathrm{m}$ vs. $20-22 \mu \mathrm{m}$ and bluntly rounded vs. spicate to pointed tail termini.

\subsection{Phylogenetic and Species Delimitation Analysis}

The D2-D3 domains of the 28S rRNA gene alignment (744 bp long) included 128 sequences of 31 Paratylenchus species and three outgroup species. Forty-nine new sequences were included in this analysis. The Bayesian $50 \%$ majority rule consensus tree inferred from the analysis of the D2-D3 alignment contained three highly supported major clades and a weakly supported one (Figure $16, \mathrm{PP}<70 \%$ ). The molecular species delimitation based on the generalized mixed-yule coalescent (GMYC) and Poisson tree process (bPTP) methods revealed 66 and 63 putative species, respectively, a result that is largely congruent with former species delineations. However, P. projectus, P. straeleni, P. minor and P. shenzhenensis were further divided into 6, 5 (four according to bPTP), 2 and 2 separate lineages, respectively.

The ITS rRNA gene alignment (995 bp long) included 99 sequences of 37 Paratylenchus species and three outgroup species. Thirty-six new sequences were included in this analysis. The Bayesian $50 \%$ majority rule consensus tree inferred from the analysis of the ITS alignment contained four highly supported major clades (Figure 17). Results of molecular species delimitation showed a high discrepancy between the models used-i.e., 48 putative species based on GMYC vs. 56 species based on bPTP. Additionally, molecular species delimitation based on the GMYC and bPTP methods did not correspond to species demarcation based on morphology and clade support; for example, virtually all individual sequences of $P$. chongqingensis and $P$. shenzhenensis were delineated as separate species. 


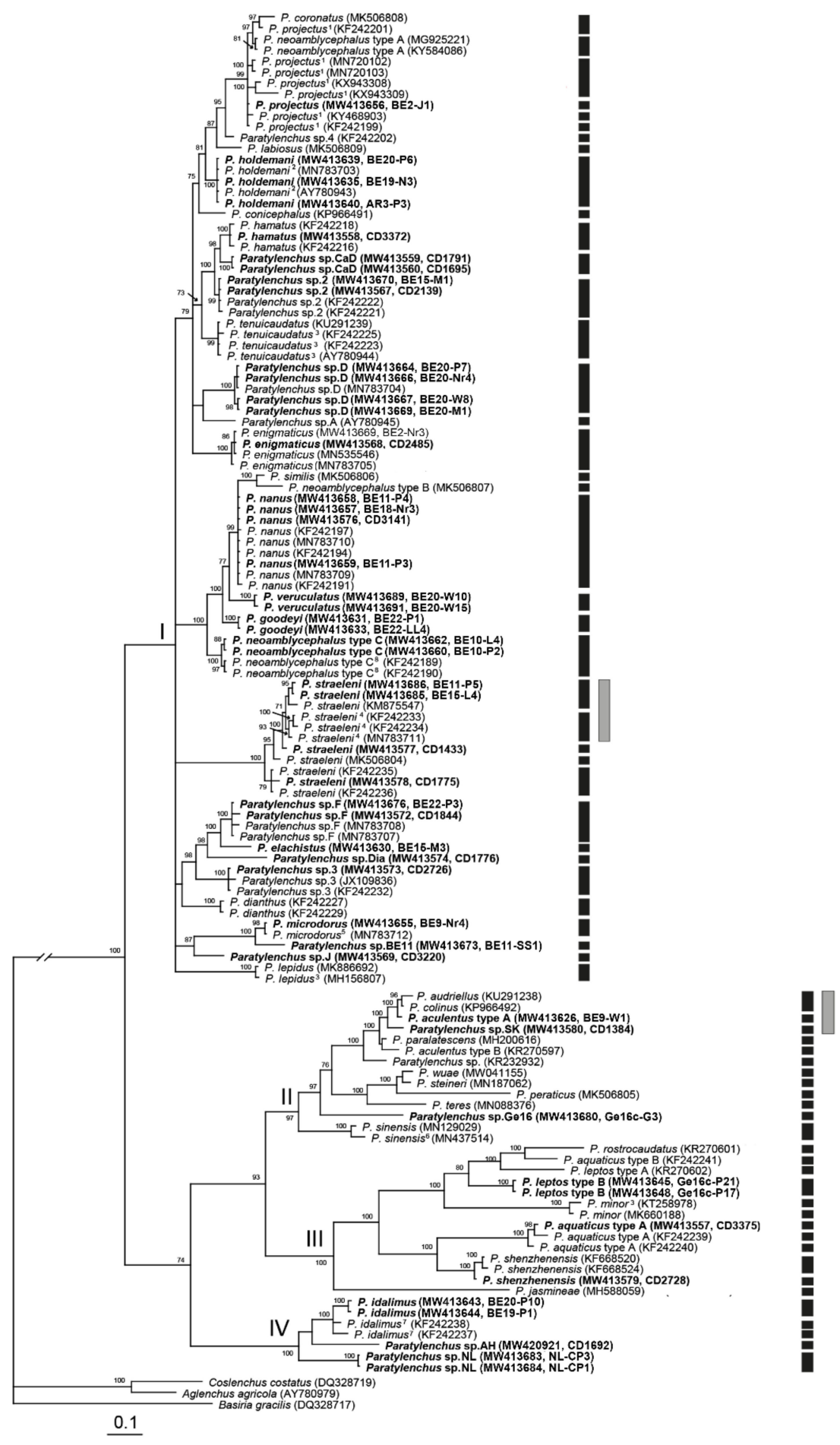

Figure 16. Phylogenetic relationships within populations and species of Paratylenchus, as inferred from Bayesian analysis using the D2-D3 of 28S rRNA gene sequence dataset with the GTR + I + G model. Posterior probability of more than $70 \%$ is given for the appropriate clades. Newly obtained sequences are indicated in bold. ${ }^{1}=$ originally identified as P. nanus, ${ }^{2}=$ originally identified as P. bukowinensis, ${ }^{3}=$ originally identified as Paratylenchus sp. ${ }^{4}=$ originally identified as Paratylenchus sp. $8,{ }^{5}=$ originally identified as Paratylenchus sp.E, ${ }^{6}=$ originally identified as Gracilacus sp. ${ }^{7}=$ originally identified as Paratylenchus sp.5 and ${ }^{8}=$ originally identified as Paratylenchus sp.6. Black and grey bars represent species boundaries estimated by generalized mixed-yule coalescent (GMYC) and Poisson tree process (bPTP) methods, respectively (only differences with GMYC provided). 


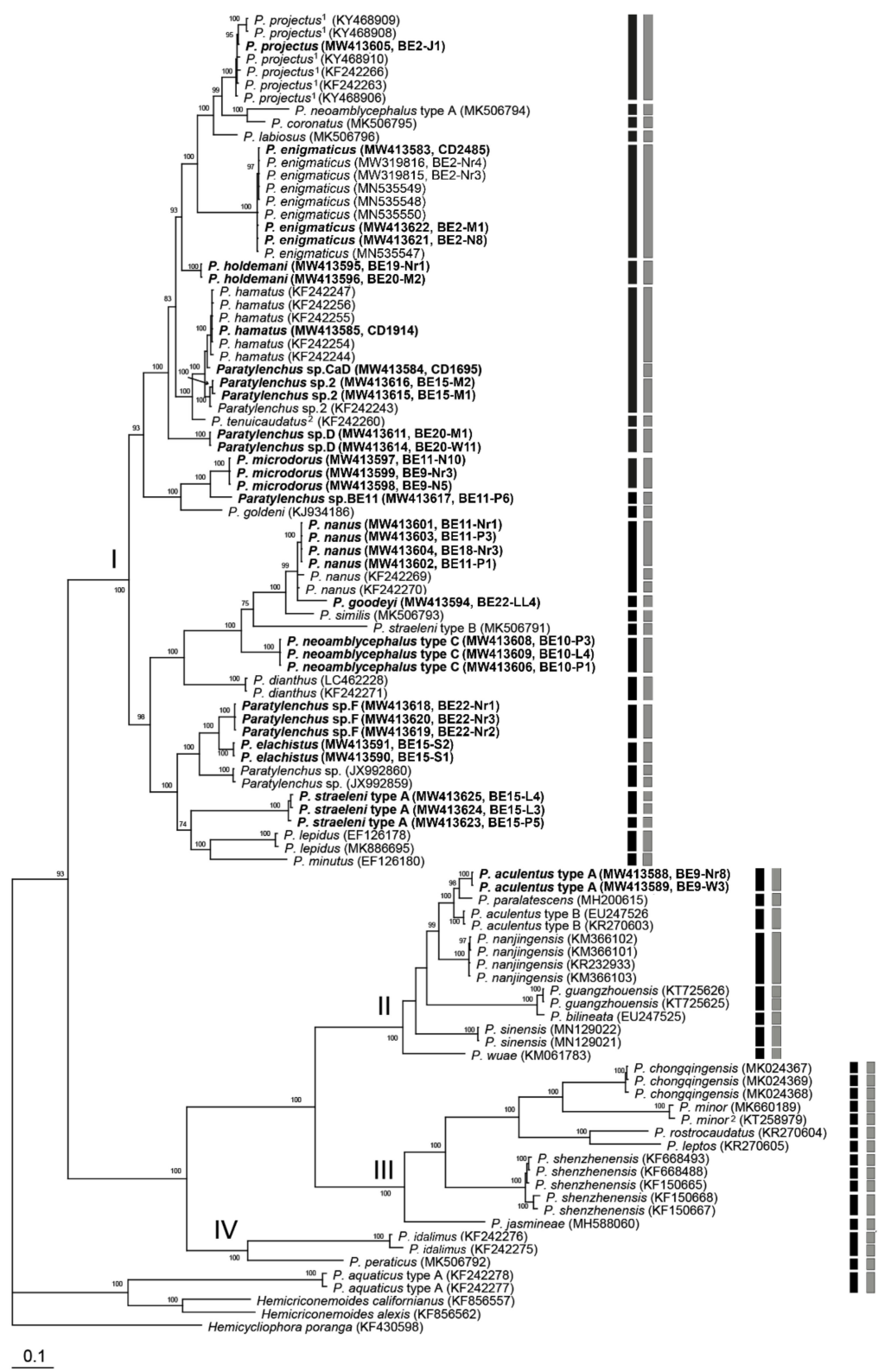

Figure 17. Phylogenetic relationships within populations and species of Paratylenchus as inferred from Bayesian analysis using the ITS rRNA gene sequence dataset with the GTR + I + G model. Posterior probability more than $70 \%$ is given for appropriate clades. Newly obtained sequences are indicated in bold. ${ }^{1}=$ originally identified as P. nanus and ${ }^{2}=$ originally identified as Paratylenchus sp. Black and grey bars represent species boundaries estimated by GMYC and bPTP methods, respectively.

The 18S rRNA gene alignment (899 bp long) included 88 sequences of 31 Paratylenchus species and two outgroup species. Fifty-four new sequences were obtained for this study. The Bayesian 50\% majority rule consensus tree inferred from the analysis of the partial $18 \mathrm{~S}$ sequence alignment contained four highly supported major clades (Figure 18). Molecular species delimitation failed to delimit well established species-for example $P$. goodeyi, $P$. veruculatus, $P$. nanus and $P$. neoamblycephalus were identified as belonging to the same species. Furthermore, both models provided highly varied results (14 putative species according to GMYC vs. 26 according to bPTP), reducing the confidence in said results. 


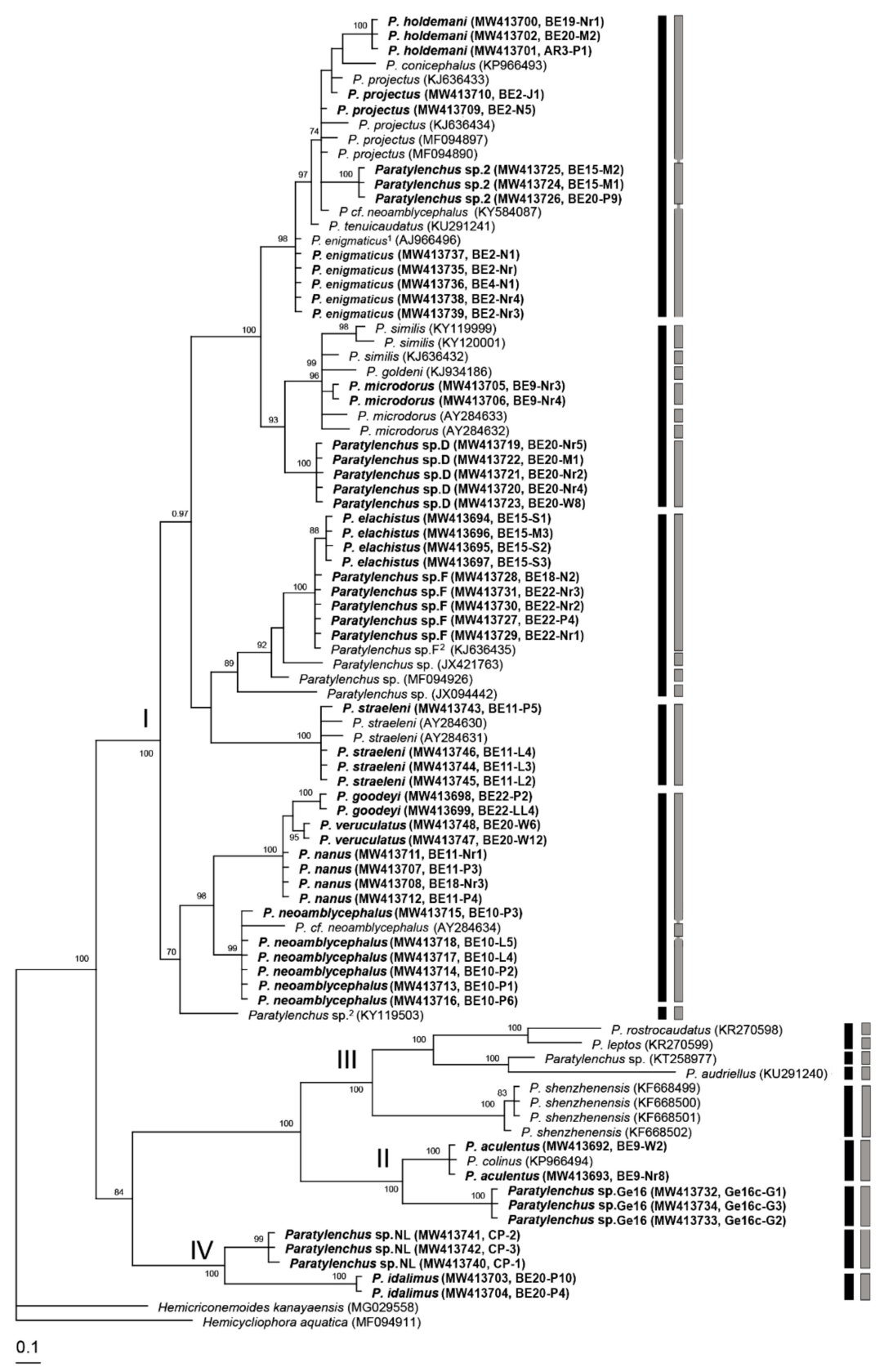

Figure 18. Phylogenetic relationships within populations and species of Paratylenchus, as inferred from Bayesian analysis using the 18S rRNA gene sequence dataset with the GTR + I + G model. Posterior probability more than $70 \%$ is given for appropriate clades. Newly obtained sequences are indicated in bold. ${ }^{1}=$ originally identified as $P$. dianthus and ${ }^{2}=$ originally identified as P. nanus. Black and grey bars represent species boundaries estimated by GMYC and bPTP methods, respectively.

The COI gene alignment (745 bp long) included 130 sequences of 31 Paratylenchus species and three outgroup species. Seventy-one new sequences were included in this analysis. The Bayesian 50\% majority rule consensus tree inferred from the analysis of the COI sequence alignment contained four moderate (Figure 19, PP $=70-90 \%$ ) or highly supported major clades. Both employed species delineation methods, GMYC and bPTP, provided exactly the same 54 putative species delineations. These results were largely consistent with those obtained using other methods. However, P. enigmaticus, P. microdorus and $P$. veruculatus were subdivided into different species despite these sequences originating from the same population and their corresponding D2-D3 sequences being similar. 
Paratylenchus straeleni was appointed as a species complex with nine putative species. Statistical parsimony networks showing the phylogenetic relationships between different isolates of P. straeleni and P. enigmaticus based on COI sequences are given in Figure 19B,C. The maximum variation of sequences for $P$. straeleni was found to be $9.1 \%$.

Taking both morphological and molecular evidence together, we have been able to reassign a total of 49 Paratylenchus sequences, including 18 D2-D3 of 28S, 3 ITS, 3 18S rRNA and 25 COI gene sequences, to their appropriate species (Table 5). However, we cannot exclude that in future, the identification of Paratylenchus species made in this study may be improved in light of new datasets.

Table 5. List of some existing unidentified or incorrectly classified Paratylenchus sequences on the GenBank reassigned to corrected species. In total, 18 D2-D3 of 28S, 3 ITS, 3 18S rRNA and 25 COI gene sequences have been reassigned.

\begin{tabular}{|c|c|c|c|c|c|}
\hline Gene & $\begin{array}{c}\text { GenBank Accession } \\
\text { No. }\end{array}$ & Linked Species & Country of Origin & Reference & $\begin{array}{c}\text { Reassigned Species } \\
\text { Name }\end{array}$ \\
\hline D2-D3 & MN437514 & Gracilacus sp. & Myanmar & Du, Y. (Unpublished) & P. sinensis \\
\hline D2-D3 & AY780943 & P. bukowinensis & Italy & Subbotin et al. [37] & P. holdemani \\
\hline D2-D3 & MN088372 & P. bukowinensis & Iran & Mirbabaei et al. [46] & P. holdemani \\
\hline D2-D3 & MN783703 & P. bukowinensis & Belgium & Etongwe et al. [47] & P. holdemani \\
\hline D2-D3 & AY780944 & Paratylenchus sp. & Italy & Subbotin et al. [37] & P. tenuicaudatus \\
\hline D2-D3 & MH156807 & Paratylenchus sp. & China & Fan et al. (Unpublished) & P. lepidus \\
\hline D2-D3 & KF242223 & Paratylenchus sp.1 & USA & Van den Berg et al. [22] & P. tenuicaudatus \\
\hline D2-D3 & KF242224 & Paratylenchus sp.1 & USA & Van den Berg et al. [22] & P. tenuicaudatus \\
\hline D2-D3 & KF242225 & Paratylenchus sp.1 & USA & Van den Berg et al. [22] & P. tenuicaudatus \\
\hline D2-D3 & KF242237 & Paratylenchus sp.5 & USA & Van den Berg et al. [22] & P. idalimus \\
\hline D2-D3 & KF242238 & Paratylenchus sp.5 & USA & Van den Berg et al. [22] & P. idalimus \\
\hline D2-D3 & KT258978 & Paratylenchus sp. & China & Liu et al. (Unpublished) & P. minor \\
\hline D2-D3 & KF242189 & Paratylenchus sp.6 & USA & Van den Berg et al. [22] & P. neoamblycephalus \\
\hline D2-D3 & KF242190 & Paratylenchus sp.6 & USA & Van den Berg et al. [22] & P. neoamblycephalus \\
\hline D2-D3 & KF242233 & Paratylenchus sp.8 & USA & Van den Berg et al. [22] & P. straeleni \\
\hline D2-D3 & KF242234 & Paratylenchus sp.8 & USA & Van den Berg et al. [22] & P. straeleni \\
\hline D2-D3 & MN783711 & Paratylenchus sp.8 & Belgium & Etongwe et al. [47] & P. straeleni \\
\hline D2-D3 & MN783712 & Paratylenchus sp.E & Belgium & Etongwe et al. [47] & P. microdorus \\
\hline ITS & KT258979 & Paratylenchus sp. & China & Liu et al. (Unpublished) & P. minor \\
\hline ITS & KF242260 & Paratylenchus sp.1 & USA & Van den Berg et al. [22] & P. tenuicaudatus \\
\hline ITS & KF242259 & Paratylenchus sp.1 & USA & Van den Berg et al. [22] & P. tenuicaudatus \\
\hline $18 \mathrm{~S}$ & AJ966496 & P. dianthus & Belgium & Meldal et al. [65] & P.enigmaticus \\
\hline $18 \mathrm{~S}$ & KJ636435 & P. nanus & The Netherlands & $\begin{array}{l}\text { Van Megen et al. } \\
\text { (Unpublished) }\end{array}$ & Paratylenchus sp.F \\
\hline $18 \mathrm{~S}$ & KY119503 & P. nanus & Ireland & Ortiz et al. [66] & Paratylenchus sp. \\
\hline $\mathrm{COI}$ & MF770960 & Gracilacus sp. & USA & $\begin{array}{l}\text { Munawar et al. } \\
\text { (Unpublished) }\end{array}$ & P. straeleni \\
\hline COI & MN710983 & Gracilacus sp. & USA & Powers et al. [49] & P. straeleni \\
\hline $\mathrm{COI}$ & MN710984 & Gracilacus sp. & USA & Powers et al. [49] & P. straeleni \\
\hline $\mathrm{COI}$ & MN711354 & Paratylenchus sp. & USA & Powers et al. [49] & P. straeleni \\
\hline COI & MN711355 & Paratylenchus sp. & USA & Powers et al. [49] & P. hamatus \\
\hline $\mathrm{COI}$ & MN711356 & Paratylenchus sp. & USA & Powers et al. [49] & P. hamatus \\
\hline $\mathrm{COI}$ & MN711357 & Paratylenchus sp. & USA & Powers et al. [49] & P. hamatus \\
\hline $\mathrm{COI}$ & MN711358 & Paratylenchus sp. & Canada & Powers et al. [49] & P. straeleni \\
\hline $\mathrm{COI}$ & MN711359 & Paratylenchus sp. & Canada & Powers et al. [49] & P. straeleni \\
\hline $\mathrm{COI}$ & MN711360 & Paratylenchus sp. & Canada & Powers et al. [49] & P. straeleni \\
\hline $\mathrm{COI}$ & MN711363 & Paratylenchus sp. & USA & Powers et al. [49] & P. straeleni \\
\hline $\mathrm{COI}$ & MN711367 & Paratylenchus sp. & Ireland & Powers et al. [49] & P. straeleni \\
\hline $\mathrm{COI}$ & MN711368 & Paratylenchus sp. & Ireland & Powers et al. [49] & P. straeleni \\
\hline $\mathrm{COI}$ & MN711369 & Paratylenchus sp. & Ireland & Powers et al. [49] & P. straeleni \\
\hline $\mathrm{COI}$ & MN711374 & Paratylenchus sp. & USA & Powers et al. [49] & P. straeleni \\
\hline $\mathrm{COI}$ & MN711375 & Paratylenchus sp. & Canada & Powers et al. [49] & P. straeleni \\
\hline $\mathrm{COI}$ & MN711376 & Paratylenchus sp. & Canada & Powers et al. [49] & P. straeleni \\
\hline $\mathrm{COI}$ & MN711378 & Paratylenchus sp. & Poland & Powers et al. [49] & P. holdemani \\
\hline $\mathrm{COI}$ & MN711380 & Paratylenchus sp. & Canada & Powers et al. [49] & P. enigmaticus \\
\hline $\mathrm{COI}$ & MN711364 & Paratylenchus sp. & Ireland & Powers et al. [49] & P. nanus \\
\hline $\mathrm{COI}$ & MN711365 & Paratylenchus sp. & Ireland & Powers et al. [49] & P. nanus \\
\hline $\mathrm{COI}$ & MN782401 & Paratylenchus sp.8 & Belgium & Etongwe et al. [47] & P. straeleni \\
\hline $\mathrm{COI}$ & MN782404 & Paratylenchus sp.B & Belgium & Etongwe et al. [47] & P. holdemani \\
\hline $\mathrm{COI}$ & MN782405 & Paratylenchus sp.B & Belgium & Etongwe et al. [47] & Paratylenchus sp.D \\
\hline $\mathrm{COI}$ & MN782406 & Paratylenchus sp.E & Belgium & Etongwe et al. [47] & P. microdorus \\
\hline
\end{tabular}




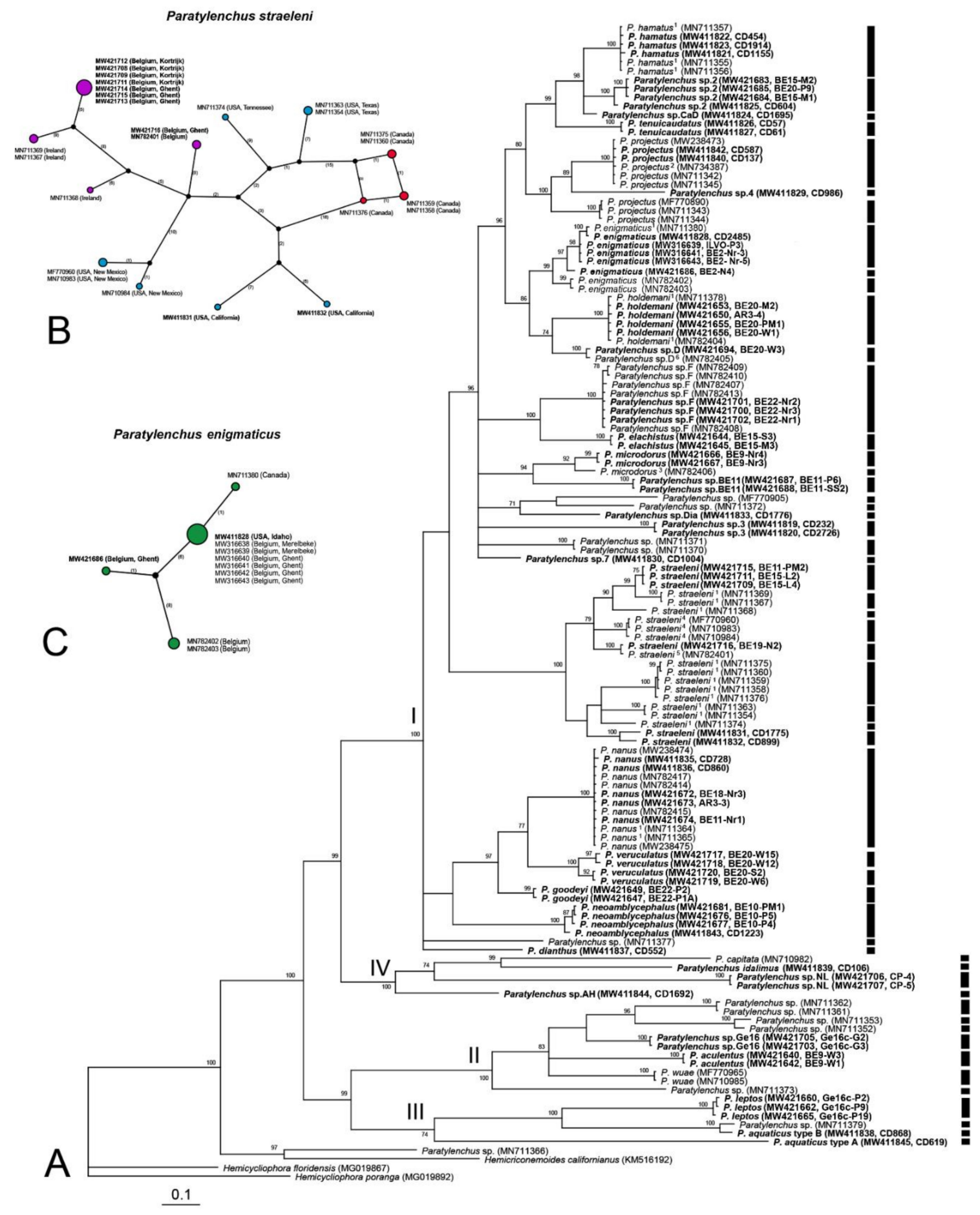

Figure 19. (A). Phylogenetic relationships within populations and species of Paratylenchus, as inferred from Bayesian analysis using the COI gene sequence dataset with the GTR + I + G model. Posterior probability more than $70 \%$ is given for appropriate clades. Newly obtained sequences are indicated in bold. ${ }^{1}=$ originally identified as Paratylenchus sp., ${ }^{2}=$ originally identified as $P$. nanus, ${ }^{3}=$ identified as Paratylenchus sp.E, ${ }^{4}=$ originally identified as Gracilacus sp., ${ }^{5}=$ originally identified as Paratylenchus sp.8, ${ }^{6}=$ originally identified as Paratylenchus sp.B; (B). Statistical parsimony network showing the phylogenetic relationships between COI haplotypes for P. straeleni; (C). Statistical parsimony network showing the phylogenetic relationships between COI haplotypes for P. enigmaticus. Pies (circles) represent the sequences with the same haplotype and their size is proportional to the number of these sequences in the samples. Numbers of nucleotide differences between the sequences are indicated on lines connecting the pies. Small black circles represent missing haplotypes. Bars represent species boundaries estimated by both GMYC and bPTP methods (identical results). 


\section{Discussion}

The genus Paratylenchus sensu lato, with 124 valid species, is an important plantparasitic group consisting of several commonly occurring and economically important species such as $P$. bukowinensis, $P$. dianthus, P. hamatus, $P$. nanus, $P$. neoamblycephalus and $P$. projectus, which are difficult to separate solely based on morphology $[14,23,25,31-36]$. Female morphological traits are the most commonly used features for the identification of Paratylenchus populations, with the relative lengths of stylet cones and the positions of the secretory-excretory pores and vulvae as the most informative traits [31,67], while several ratios such as a, c and c' show high intraspecific variation. Given the limited species-specific female traits, some characteristics of males and juveniles-such as the presence or absence of stylet and male spicule length - may also be used to supplement the available data. However, care must be taken-for example, the occasional observance of a thin stylet in freshly killed juveniles or males that was invisible once the specimens were fixed highlights the importance of reporting this characteristic from both freshly killed and fixed specimens. Further complicating Paratylenchus taxonomy is the presence of mixture of species within one locality and sample [31] —an observation which calls for precaution concerning the conspecificity of several life stages. Indeed, the presence of multiple species in a soil sample was amply illustrated in our study. Seventy five percent of our investigated soil samples contained multiple species, with up to five different Paratylenchus species present in the same sample. This is, to the best of our knowledge, one of the highest numbers of species of one plant-parasitic nematode genus present in a single soil sample. More suitable morphological characters such as ridges around the oral opening or distinct to fused submedian lobes in face view also appear to be usefully informative but were only clearly revealed in our study with supporting evidence from SEM; additionally, the small vulval flaps in P. aculentus confirmed in this study have often been overlooked in previous studies under LM. Scanning electron microscopy is known to be important in nematode taxonomy [68-70], and this is especially true for the genus Paratylenchus as demonstrated in this study.

Nevertheless, even if all existing morphological tools are carefully employed, it remains impossible for all Paratylenchus species to be morphologically delineated, owing to the existence of cryptic species such as P. aquaticus [22]. The extensive use of new molecular data in the current study has demonstrated a remarkable molecular diversity in Paratylenchus, with several additional cryptic species being potentially present. The most obvious example is $P$. straeleni, which comprises 9,5 and 4 putative species according to COI- (both GMYC and bPTP), D2-D3 (GMYC) and D2-D3 (bPTP)-based molecular species delimitation methods, respectively. It is noteworthy that the P. straeleni COI sequences have clearly clustered according to geographical location, as revealed by the COI haplotype network. The problems of morphologically delineating the Paratylenchus species have been further demonstrated in our study by the difficulties experienced in distinguishing between Paratylenchus sp.2, Paratylenchus sp.D and Paratylenchus sp.F, which were found to be very similar to $P$. hamatus, $P$. projectus / P. neoprojectus and $P$. nanus, respectively. A formal description with an appropriate diagnosis can only be developed for these putative new species following detailed observations of additional specimens and a thorough comparison with type materials of the known species.

Taken together, it is abundantly clear that molecular data are essential in advancing Paratylenchus taxonomy. Unfortunately, the several sequences published for Paratylenchus have serious limitations. One such issue is that the majority of the available D2-D3, 18S and ITS rRNA sequences have either not been linked to morphological data or have been associated with poor morphological data, thereby rendering them unreliable for use in identification purposes. For example, sequences of P. aculentus, P. leptos, P. microdorus, $P$. neoamblycephalus, etc., are not currently linked to reliable and clear morphological data and any subsequent identification based on these sequences may, therefore, lead to the deposition of further sequences under incorrect names [51]. An additional problem identified with the currently available $18 \mathrm{~S}$ sequences, which are often relatively short (700-800 bp), is 
that several Paratylenchus species were detected with almost identical sequences. It is clear that to render these conserved sequences useful, complete or nearly complete lengths of the $18 \mathrm{~S}$ rRNA gene (1600-1800 bp) will be required to allow species delimitation [71-74].

In the present study, we have also applied DNA-based species delimitation approaches to infer putative species boundaries on a given phylogenetic input, based on two different models $[75,76]$ and four gene fragments (D2-D3 of 28S, ITS, $18 \mathrm{~S}$ rRNA and COI). These coalescence-based species delimitation methods are rapidly gaining popularity in studies on closely related species that are difficult to distinguish based on phenotypic features, and have been applied to various eukaryotic groups [77,78]. However, despite plant-parasitic nematodes being a morphologically minimalistic group par excellence, such methods have been rarely applied to this group; nevertheless, they appear to be largely congruent with traditional methods [79-81]. Conversely, we have observed a remarkable discrepancy among the genes used, showing a poor link between DNA species delimitation and other methods, including a discrepancy between the employed models. The ITS and 18S rRNA genes gave, respectively, a likely overestimation and underestimation of the number of putative species, while for COI and D2-D3 of 28S rRNA genes, we observed, to a certain extent, an agreement with traditional methods, albeit with a likely overestimation of the number of species in several cases. This was not unexpected, as it has been exemplified by several studies that methods of species delimitation based on the coalescent model tend to overestimate phylogenetic lineages $[52,77,82]$. Both approaches (bPTP and GMYC) are similar in the fact that they identify significant changes in the pace of branching events on the tree. However, GMYC uses time to identify branching rate transition points, whereas, in contrast, bPTP directly uses the number of substitutions. Based on real and simulated data, both methods yield, in general, similar results $[76,83]$. This is the case for our COI-based output (identical results) and the D2-D3-based output (two differences, bPTP being more conserved). If differences have been observed, bPTP usually yields a more conservative delimitation than GMYC $[76,80,83]$. This is contrary to our unexpected ITS and $18 \mathrm{~S}$ results and reduces the trust in the latter. Counterintuitively, the mutation rate of a chosen marker does not have a direct influence on its effectiveness to detect species. Mitochondrial markers reveal clearer discontinuities between interspecific divergence and intraspecific variation because of their faster coalescence within species lineages compared with nuclear loci, not necessarily because of their higher mutation rates $[84,85]$. The discrepancy between ITS and other delimitation methods in this study agrees with previous observations pointing to an unclear transition between species-level and population-level genetic distance for ITS [78]. Furthermore, it has been indicated that species delimitation based on single gene trees has serious limitations due to gene tree-species tree incongruence-confusions caused by processes including incomplete lineage sorting, trans-species polymorphism, hybridisation and introgression [78]. Multilocus approaches provide a posteriori double-check for contamination, sequencing errors or mitochondria-specific pitfalls [86] — for example, the high COI gene sequence variations within $P$. enigmaticus, $P$. microdorus and $P$. veruculatus observed in this study, despite these sequences originating from the same population. Although both nuclear and mitochondrial sequences were provided consistently from the same morphologically vouchered individuals, this study was restricted to the use of only single-locus data since only a limited number of other Paratylenchus individuals (and plant-parasitic nematodes in general) are linked to the same two genes. A further rigorous acquisition of both D2-D3 of 28S and COI gene sequences, which appear most promising for species delimitation in plant-parasitic nematodes (see [74]), will allow for more substantiated coalescence-based, multilocus species delimitation in plant-parasitic nematodes. Nevertheless, based on all obtained evidence, our findings support the proposition of Puillandre et al. [87], Padial et al. [88] and Qing et al. [80], that DNA-based species delimitation methods are important tools for the exploration of species delineation in diverse groups, but that identification of any new putative species will require further corroboration by an integrative taxonomic approach. 


\section{Materials and Methods}

\subsection{Nematode Populations}

Nematode samples used in this study were collected from various localities (Table 1). Bulk soil samples of about $500 \mathrm{~mL}$ from 15-20 cm depths were collected from twelve locations in Belgium using a shovel. They were subsequently stored at $4{ }^{\circ} \mathrm{C}$ until nematode extraction. Nematodes were extracted from soil using a modified Baermann's method [89] or a rapid centrifugal flotation method [90]. Nematode extracts were observed under a stereo microscope. Paratylenchus populations were picked out in an embryo glass dish and stored in tap water at $4{ }^{\circ} \mathrm{C}$ for further analysis.

\subsection{Morphological Study}

Morphological study of nematodes was carried out using both heat relaxed and fixed specimens mounted on temporary and permanent slides, respectively. For preparation of a temporary mount of a nematode, a Cryo-Pro label (VWR International) was cut into two halves and stuck at the centre of a glass slide creating a small parallel gap between them. A single nematode was then transferred in a drop of distilled water to the glass slide in the centre of the gap. The nematode was then heat relaxed by passing over a flame a few times and covered with a glass coverslip. The specimen was then examined, photographed and measured using an Olympus BX51 DIC Microscope (Olympus Optical, Tokyo, Japan), equipped with an Olympus C5060Wz camera [91]. After recording morphological data, the specimen was recovered from the slide by adding a few drops of water from one end of the gap and collecting the nematode that was flushed out on the other end of the gap. The recovered specimens were subsequently used to extract genomic DNA as described in the next section.

A small nematode suspension of the remaining nematodes was heated in an embryo glass dish with a few drops of Trump's fixative (( $2 \%$ paraformaldehyde, $2.5 \%$ glutaraldehyde in $0.1 \mathrm{M}$ Sorenson buffer (sodium phosphate buffer at $\mathrm{pH}=7.5$ )) in a microwave (700 Watts) for 3-4 sec and leaving it at room temperature for $1 \mathrm{~h}$ and at $4{ }^{\circ} \mathrm{C}$ for $24 \mathrm{~h}$ and followed by gradually transferring to anhydrous glycerine, as described in Singh et al. [92]. The fixed specimens were then mounted in glycerine on glass slides and were studied as above using the camera-equipped microscope. Species identification was carried out both at Nematology Research Unit of Ghent University and National Plant Protection Organization, Wageningen, the Netherlands.

For scanning electron microscopy, specimens fixed in Trump's fixative were washed in $0.1 \mathrm{M}$ phosphate buffer $(\mathrm{pH}=7.5)$ and dehydrated in a graded series of ethanol solutions, critical point-dried with liquid $\mathrm{CO}_{2}$, mounted on stubs with carbon tabs (double conductive tapes), coated with gold of $25 \mathrm{~nm}$, and photographed with a JSM-840 EM (JEOL) at $12 \mathrm{kV}$ [92].

\subsection{Extraction of DNA, PCR and Sequencing}

Genomic DNA was extracted from individual heat relaxed nematode specimen, which had been morphologically vouchered. The cuticle of the specimen was punctured using a fine entomological pin mounted on a thin bamboo stick, which was also used as nematode picking tool and the nematode was subsequently transferred to a PCR tube with $20 \mu \mathrm{L}$ of worm lysis buffer $\left(50 \mathrm{mM} \mathrm{KCl}, 10 \mathrm{mM}\right.$ Tris at $\mathrm{pH}=8.3,2.5 \mathrm{mM} \mathrm{MgCl}_{2}, 0.45 \% \mathrm{NP} 40$ (Tergitol Sigma), $0.45 \%$ Tween 20 ) and incubated at $-20{ }^{\circ} \mathrm{C}$ (at least $10 \mathrm{~min}$ ). This was followed by adding $1 \mu \mathrm{L}$ proteinase $\mathrm{K}(1.2 \mathrm{mg} / \mathrm{mL})$, incubation at $65^{\circ} \mathrm{C}(1 \mathrm{~h})$ and $95^{\circ} \mathrm{C}(10 \mathrm{~min})$ and ending by centrifuging the mixture at 14,000 rpm for $1 \mathrm{~min}$ [92]. Genomic DNA from a single nematode was used to amplify four DNA fragments-D2-D3 of 28S, partial ITS and partial $18 \mathrm{~S}$ rRNA gene and partial COI gene of mtDNA. PCR and sequencing were completed in two laboratories: Nematology Research Unit, Gent University, Belgium and Nematology lab, Plant Pest Diagnostic Center, CDFA, Sacramento, California, USA. For PCR amplifications of the D2-D3 of 28S, ITS and 18S rRNA gene sequences, the primer pairs D2A: 5'-ACA AGT ACC GTG AGG GAA AGT TG-3' / D3B: 5'-TCC TCG GAA GGA 
ACC AGC TAC TA-3' [93], Vrain2F: 5'-CTT TGT ACA CAC CGC CCG TCG CT-3' / Vrain2R: 5'-TTT CAC TCG CCG TTA CTA AGG GAA TC-3' [94] or TW81: $5^{\prime}$-GTT TCC GTA GGT GAA CCT GC-3' / AB28: $5^{\prime}$-ATA TGC TTA AGT TCA GCG GGT-3' [95], and SSU18A: 5'-AAA GAT TAA GCC ATG CAT G-3' /SSU26R: 5'-CAT TCT TGG CAA ATG CTT TCG$3^{\prime}$ [96] were used, respectively, with thermal profiles described by Singh et al. [97] and Tahna Maafi et al. [98]. Partial COI gene was amplified using the primer pairs JB3: $5^{\prime}$-TTT TTT GGG CAT CCT GAG GTT TAT-3' /JB4.5: 5'-TTT TTT GGG CAT CCT GAG GTT TAT-3' according to Bowles et al. [99] or COI-F5: 5'-AAT WTW GGT GTT GGA ACT TCT TGA AC-3'/COI-R9: 5'-CTT AAA ACA TAA TGR AAA TGW GCW ACW ACA TAA TAA GTA TC- $3^{\prime}$ according to Powers et al. [100]. The PCR products were purified [101] and sent to Macrogen [102] and Genewiz [103] for sequencing. New sequences were assembled using Geneious Prime 2020.0.5 and deposited to the GenBank under the accession numbers given in Table 1.

\subsection{Phylogenetic and Species Delimitation Analysis}

The new sequences for each gene (D2-D3 of 28S, ITS, 18S rRNA and COI) were aligned using Clustal $X \quad 1.83$ [104] with their corresponding published gene sequences $[22,24,32-39,42-50]$. Outgroup taxa for each dataset were chosen based on previously published data [105]. Sequence datasets were analysed with Bayesian inference (BI) using MrBayes 3.1.2 [106] under the GTR + I + G model. BI analysis was initiated with a random starting tree and was run with four chains for $1.0 \times 10^{6}$ generations for $18 \mathrm{~S}$ and ITS rRNA gene alignments, $5.0 \times 10^{6}$ generations for D2-D3 of 28S rRNA gene alignment and $9.0 \times 10^{6}$ generations for COI gene alignment. The Markov chains were sampled at intervals of 100 generations. Two runs were performed for each analysis. The log-likelihood values of the sample points stabilised after approximately 1,000 generations. After discarding burn-in samples and evaluating convergence, the remaining samples were retained for further analysis. The topologies were used to generate a $50 \%$ majority rule consensus tree. Posterior probabilities (PPs) are given on appropriate clades. Sequence analyses of alignments were performed with PAUP* $4 \mathrm{~b} 10$ [107]. Pairwise divergences between taxa were computed as absolute distance values and as percentage mean distance values based on whole alignment with adjustments for missing data.

The COI gene alignments for $P$. straeleni and P. enigmaticus were used to construct phylogenetic network estimation using statistical parsimony, as implemented in POPART software [108].

Species delimitation of Paratylenchus in this study was undertaken using an integrated approach that considered morphological and morphometric evaluations combined with molecular-based phylogenetic inference (tree-based methods) and coalescent-based molecular species-delimitation methods. Putative species boundaries on a given phylogenetic input tree were inferred using a Bayesian implementation of the Poisson tree processes (bPTP) method [76] and using the generalized mixed-yule coalescent (GMYC) method [75]; see Qing et al. [80] for more details. Ultrametric trees were constructed using BEAST v1.10.4 [109] based on D2-D3, ITS, 18S and COI sequences, respectively. Default prior distributions were used and analyses were run for $1 \times 10^{7}$ generations, saving trees every $1 \times 10^{3}$ generations. The final trees were produced after removing 2,000 samples $(20 \%)$ as burn-ins, and the maximum clade credibility tree was calculated using TreeAnnotator 1.10.4 [109]. Finally, for the bPTP method, an unrooted Bayesian 50\% majority-rule consensus tree, containing only ingroups and unique haplotypes, was uploaded on the online server [110] and $1 \times 10^{5}$ Markov chain Monte Carlo (MCMC) generations were performed. The same tree was also uploaded on the GMYC web server [111] using the single threshold method.

\section{Conclusions}

An integrative approach by linking DNA sequences and morphological characters represents the best way to move nematode taxonomy forward. Creating this link involves 
the rigorous generation of multiple DNA sequences from individual morphologically vouchered nematode specimens, which, in the current study, resulted in the first molecular characterisations for five species, the first $\mathrm{COI}$ sequences for eight species and, most importantly, the reassignments of 18 D2-D3 of 28S, 3 ITS, 318 rRNA and 25 COI gene sequences, which had been unidentified or misidentified.

This study showed that Paratylenchus is a case in point, representing an incredibly diverse yet morphologically minimalistic plant-parasitic genus. Our recommendations for future protocol in Paratylenchus taxonomy, which are also valid for integrative nematode taxonomy, are: (1) to include SEM in new descriptions or re-descriptions; (2) to use juvenile and male traits after their conspecificity is irrefutably proven using molecular data; (3) to unequivocally link elaborate morphological data with both nuclear D2-D3 of 28S rRNA and mitochondrial COI gene sequences; (4) to employ caution when performing molecular identification using partial $18 \mathrm{~S}$ rRNA gene fragments only; (5) to make use of the promising molecular species delineation methods to establish species boundaries, but base this on multilocus data and merely use it as one of the elements of integrative taxonomy.

Author Contributions: Conceptualisation, P.R.S., W.B. and S.A.S.; methodology, P.R.S., M.C., W.B. and S.A.S.; software, P.R.S., W.B. and S.A.S.; validation, P.R.S., G.K., W.B. and S.A.S.; formal analysis, P.R.S., G.K., W.B. and S.A.S.; investigation, P.R.S., G.K., W.B. and S.A.S.; resources, P.R.S., G.K., M.C., W.B. and S.A.S.; data curation, P.R.S., G.K. and S.A.S.; writing-original draft preparation, P.R.S.; writing - review and editing, W.B., S.A.S. and G.K.; visualisation, P.R.S. and M.C.; supervision, W.B. and G.K.; project administration, W.B.; funding acquisition, W.B. and S.A.S. All authors have read and agreed to the published version of the manuscript.

Funding: This research was funded by Ghent University, Belgium, UGent BOF01D05918 and the Plant Pest Diagnostic Center, CDFA, USA.

Institutional Review Board Statement: Not applicable.

Informed Consent Statement: Not applicable.

Data Availability Statement: The datasets generated during and/or analyzed during the currentstudy are available from the corresponding author on reasonable request.

Acknowledgments: The authors thank E. Van den Berg, A. Westphal, J. Zogfar, C.M. Etongwe and A.W. Aseffa for providing nematode specimens and soil samples.

Conflicts of Interest: The authors declare no conflict of interest.

\section{References}

1. Micoletzky, H. Die freilebenden Erd-Nematoden; Archiv für Naturgeschichte: Berlin, Germany, 1922.

2. Siddiqi, M.R. Tylenchida: Parasites of Plants and Insects, 2nd ed.; CABI Publishing: Wallingford, UK, 2000.

3. Manzanilla-López, R.H.; Marbán-Mendoza, N. Practical plant nematology; BBA: Colegio de Postgraduados, Mexico, 2012.

4. Fourie, H.; Spaull, V.W.; Jones, R.K.; Daneel, M.S.; De Waele, D. Nematology in South Africa: A View from the 21st Century; Springer: Cham, Switzerland, 2017.

5. Subbotin, S.A.; Chitambar, J.J. Plant Parasitic Nematodes in Sustainable Agriculture of North America; Springer: Cham, Switzerland, 2018

6. Tarjan, A.C. A review of the genus Paratylenchus Micoletzky, 1922 (Paratylenchinae: Nematoda) with a description of two new species. Ann. N. Y. Acad. Sci. 1960, 84, 329-390. [CrossRef]

7. Raski, D.J. Paratylenchidae n. fam. with descriptions of five new species of Gracilacus n. g. and an emendation of Cacopaurus Thorne, 1943, Paratylenchus Micoletzky, 1922 and Criconematidae Thorne, 1943. Proc. Helm. Soc. Wash. 1962, $29,189-207$.

8. Thorne, G.; Malek, R.B. Nematodes of the Northern Great Plains part I. Tylenchida [Nemata Secernentra]; South Dakota State University, Brookings: Sioux Falls, SD, USA, 1968.

9. Raski, D.J.; Luc, M. A reappraisal of Tylenchina (Nemata). 10: The superfamily Criconematoidea Taylor, 1936. Rev. Nématol. 1987, 10, 409-444.

10. Maggenti, A.R.; Luc, M.; Raski, D.J.; Fortuner, R.; Geraert, E. A reappraisal of Tylenchina (Nemata). 11. List of generic and supra-generic taxa, with their junior synonyms. Rev. Nématol. 1988, 11, 177-188.

11. Raski, D.J. Tylenchulidae of agricultural soils. In Manual of Agricultural Nematology; Nickle, W.R., Ed.; Marcel Dekker Inc.: New York, NY, USA, 1991; pp. 761-794.

12. Esser, R.P. A diagnostic compendium to species included in Paratylenchinae Thorne, 1949 and Tylenchocriconematinae Raski \& Siddiqi, 1975 (Nematoda: Criconematoidea). Nematologica 1992, 38, 146-163. 
13. Andrássy, I. Free-living nematodes of Hungary, II (Nematoda, Errantia); Hungarian Natural History Museum: Budapest, Hungary, 2007.

14. Yu, Q.; Ye, W.; Powers, T. Morphological and Molecular Characterization of Gracilacus wuae n. sp. (Nematoda: Criconematoidea) Associated with Cow Parsnip (Heracleum maximum) in Ontario, Canada. J. Nematol. 2016, 48, 203-213. [CrossRef]

15. Siddiqi, M.R. Tylenchida: Parasites of Plants and Insects; Commonwealth Agricultural Bureaux: Slough, UK, 1986.

16. Brzeski, M.W.; Szczygiel, A. Studies On the Nematodes of the Genus Paratylenchus Micoletzky (Nematoda: Paratylenchinae) in Poland. Nematologica 1963, 9, 613-625. [CrossRef]

17. Siddiqi, M.R.; Goodey, J.B. The status of the genera and subfamilies of the Criconematidae (Nematoda); with a comment on the position of Fergusobia. Nematologica 1964, 9, 363-377. [CrossRef]

18. Geraert, E. The genus Paratylenchus. Nematologica 1965, 11, 301-334. [CrossRef]

19. Brzeski, M.W. Nematodes of Tylenchina in Poland and temperate Europe; Muzeum i Instytut Zoologii, Polska Akademia Nauk: Warszawa, Poland, 1998.

20. Nguyen, C.N.; Baldwin, J.G.; Choi, Y.E. New records of Paratylenchus Micoletzky, 1922 (Nematoda: Paratylenchinae) from Viet Nam with description of Paratylenchus laocaiensis sp. n. J. Nematode Morphol. Syst. 2004, 7, 51-75.

21. Decraemer, W.; Hunt, D.J. Structure and classification. In Plant nematology; Perry, R.N., Moens, M., Eds.; CABI Publishing: Wallingford, UK, 2006; pp. 3-32.

22. Van den Berg, E.; Tiedt, L.R.; Subbotin, S.A. Morphological and molecular characterisation of several Paratylenchus Micoletzky, 1922 (Tylenchida: Paratylenchidae) species from South Africa and USA, together with some taxonomic notes. Nematology 2014, 16, 323-358. [CrossRef]

23. Ghaderi, R.; Kashi, L.; Karegar, A. Contribution to the study of the genus Paratylenchus Micoletzky, 1922 sensu lato (Nematoda: Tylenchulidae). Zootaxa 2014, 3841, 151-187. [CrossRef]

24. Hesar, A.M.; Karegar, A.; Ghaderi, R. Phylogenetic relationships of Cacopaurus pestis Thorne, 1943 within representatives of the Tylenchulidae Skarbilovich, 1947 as inferred from ITS and D2-D3 expansion segments of 28S-rRNA sequences. Nematology 2019, 21, 971-994. [CrossRef]

25. Maria, M.; Miao, W.; Castillo, P.; Zheng, J. A new pin nematode, Paratylenchus sinensis n. sp. (Nematoda: Paratylenchinae) in the rhizosphere of white mulberry from Zhejiang Province, China. Eur. J. Plant Pathol. 2020, 156, 1023-1039. [CrossRef]

26. Raski, D.J. Paratylenchoides gen. n. and two new species (Nematoda: Paratylenchidae). Proc. Helm. Soc. Wash. 1973, 40, $230-233$.

27. Ganguly, S.; Khan, E. Gracilpaurus corbetti gen. n., sp. n. (Nematoda: Tylenchida: Paratylenchidae) from India with a key to the species of this genus. Pak. J. Nematol. 1990, 8, 65-71.

28. Thorne, G. Cacopaurus pestis, n. g., n. spec. (Nematoda: Criconematinae), a destructive parasite of the walnut, Juglans regia Linn. Proc. Helm. Soc. Wash. 1943, 10, 78-83.

29. Goodey, T. Soil and Freshwater Nematodes, 2nd ed.; Goodey, J.B., Ed.; Methuen: London, UK, 1963; p. 544.

30. Ebsary, B.A. Catalog of the Order Tylenchida (Nematoda); Agriculture Canada, Research Branch: Ottawa, ON, Canada, 1991.

31. Ghaderi, R.; Geraert, E.; Karegar, A. The Tylenchulidae of the world: Identification of the family Tylenchulidae (Nematoda: Tylenchida); Academia Press: Ghent, Belgium, 2016.

32. Wang, K.; Li, Y.; Xie, H.; Xu, C.L.; Wu, W.J. Morphology and molecular analysis of Paratylenchus guangzhouensis n. sp. (Nematoda: Paratylenchinae) from the soil associated with Bambusa multiplex in China. Eur. J. Plant Pathol. 2016, 145, 255-264. [CrossRef]

33. Wang, K.; Xie, H.; Li, Y.; Wu, W.J.; Xu, C.L. Morphology and molecular analysis of Paratylenchus nanjingensis n. sp. (Nematoda: Paratylenchinae) from the rhizosphere soil of Pinus massoniana in China. J. Helminthol. 2016, 90, 166-173. [CrossRef]

34. Maria, M.; Cai, R.; Ye, W.; Powers, T.O.; Zheng, J. Description of Gracilacus paralatescens n. sp. (Nematoda: Paratylenchinae) found from the rhizosphere of Bamboo in Zhejiang, China. J. Nematol. 2018, 50, 611-622. [CrossRef]

35. Li, Y.; Wang, K.; Xie, H.; Xu, C.L. Morphology and molecular analysis of Paratylenchus chongqingensis n. sp. (Nematoda: Paratylenchinae) from soil associated with Ophiopogon japonicus in China. Eur. J. Plant Pathol. 2019, 154, 597-605. [CrossRef]

36. Phani, V.; Somvanshi, V.S.; Rao, U.; Khan, M.R. Paratylenchus jasmineae sp. n. (Nematoda: Paratylenchinae) from rhizosphere of Jasminum sambac in India. Nematology 2019, 21, 469-478. [CrossRef]

37. Subbotin, S.A.; Vovlas, N.; Crozzoli, R.; Sturhan, D.; Lamberti, F.; Moens, M.; Baldwin, J.G. Phylogeny of Criconematina Siddiqi, 1980 (Nematoda: Tylenchida) based on morphology and D2-D3 expansion segments of the 28SrRNA gene sequences with application of a secondary structure model. Nematology 2005, 7, 927-944.

38. Chen, D.Y.; Ni, H.F.; Tsay, T.T.; Yen, J.H. Identification of Gracilacus bilineata and G. aculenta (Nematoda: Criconematoidea, Tylenchulidae) among bamboo plantations in Taiwan. Plant Pathol. Bull. 2008, 17, 209-219.

39. Chen, D.Y.; Ni, H.F.; Yen, J.H.; Tsay, T.T. Identification of a new recorded pin nematode Paratylenchus minutus (Nematoda: Criconematoidea, Tylenchulidae) in Taiwan. Plant Pathol. Bull. 2009, 18, 167-174.

40. van Megen, H.; van den Elsen, S.; Holterman, M.; Karssen, G.; Mooyman, P.; Bongers, T.; Holovachov, O.; Bakker, L.; Helder, J. A phylogenetic tree of nematodes based on about 1200 full-length small subunit ribosomal DNA sequences. Nematology 2009, 11, 927-950. [CrossRef]

41. López, M.A.C.; Robbins, R.T.; Szalanski, A.L. Taxonomic and molecular identification of Hemicaloosia, Hemicycliophora, Gracilacus and Paratylenchus species (Nematoda: Criconematidae). J. Nematol. 2013, 45, 145-171.

42. Akyazi, F.; Felek, A.F.; Čermák, V.; Čudejková, M.; Foit, J.; Yildiz, S.; Háněl, L. Description of Paratylenchus (Gracilacus) straeleni (De Coninck, 1931) Oostenbrink, 1960 (Nematoda: Criconematoidea, Tylenchulidae) from hazelnut in Turkey and its comparison with other world populations. Helminthologia 2015, 52, 270-279. [CrossRef] 
43. Esmaeili, M.; Heydari, R.; Castillo, P.; Bidhendi, M.Z.; Palomares-Rius, J.E. Molecular characterisation of two known species of Paratylenchus Micoletzky, 1922 from Iran with notes on the validity of Paratylenchus audriellus Brown, 1959. Nematology 2016, 18, 591-604. [CrossRef]

44. Zhuo, K.; Liu, X.; Tao, Y.; Wang, H.; Lin, B.; Liao, J. Morphological and molecular characterisation of three species of Paratylenchus Micoletzky, 1922 (Tylenchida: Paratylenchidae) from China, with a first description of the male P. rostrocaudatus. Nematology 2018, 20, 837-850. [CrossRef]

45. Maria, M.; Miao, W.; Ye, W.; Zheng, J. Updated description of Paratylenchus lepidus Raski 1975 and P. minor Sharma, Sharma and Khan, 1986 by integrating molecular and ultra-structural observations. J. Nematol. 2019, 51, 1-13. [CrossRef]

46. Mirbabaei, H.; Eskandari, A.; Ghaderi, R. On the synonymy of Trophotylenchulus asoensis and T. okamotoi with T. arenarius, and intra-generic structure of Paratylenchus (Nematoda: Tylenchulidae). J. Nematol. 2019, 51, 1-22. [CrossRef]

47. Etongwe, C.M.; Singh, P.R.; Bert, W.; Subbotin, S.A. Molecular characterisation of some plant-parasitic nematodes (Nematoda: Tylenchida) from Belgium. Russ. J. Nematol. 2020, 28, 1-28.

48. Mwamula, A.O.; Kabir, M.F.; Lee, G.; Choi, I.H.; Kim, Y.H.; Bae, E.J.; Lee, D.W. Morphological characterisation and molecular phylogeny of several species of Criconematina (Nematoda: Tylenchida) associated with turfgrass in Korea, as inferred from ribosomal and mitochondrial DNA. Nematology 2020, 22, 939-956. [CrossRef]

49. Powers, T.; Harris, T.S.; Higgins, R.S.; Mullin, P.G.; Powers, K.S. Nematode biodiversity assessments need vouchered databases: A BOLD reference library for plant-parasitic nematodes in the superfamily Criconematoidea. Genome 2020, 5, 1-10. [CrossRef] [PubMed]

50. Wang, W.H.; Chen, P.J. First Report of a Pin Nematode (Paratylenchus dianthus) on Chrysanthemum in Taiwan. Plant Dis. 2020, 104, 995. [CrossRef]

51. Janssen, T.; Karssen, G.; Couvreur, M.; Waeyenberge, L.; Bert, W. The pitfalls of molecular species identification: A case study within the genus Pratylenchus (Nematoda: Pratylenchidae). Nematology 2017, 19, 1179-1199. [CrossRef]

52. Palomares-Rius, J.E.; Cantalapiedra-Navarrete, C.; Castillo, P. Cryptic species in plant-parasitic nematodes. Nematology 2014, 16, 1105-1118. [CrossRef]

53. Bert, W.; Geraert, E. Nematode species of the order Tylenchida, new to the Belgian nematofauna with additional morphological data. Belg. J. Zool. 2000, 130, 47-57.

54. Brzeski, M.W. Paratylenchinae: Morphology of some known species and descriptions of Gracilacus bilineata sp. n. and G. vera sp. n. (Nematoda: Tylenchulidae). Nematologica 1995, 41, 535-565. [CrossRef]

55. Lišková, M.; Sasanelli, N.; D'Addabbo, T. Some notes on the occurrence of plant parasitic nematodes on fruit trees in Slovakia. Plant Prot. Sci. 2007, 43, 26-32. [CrossRef]

56. Steiner, G. Plant nematodes the grower should know. Proc. Soil Sci. Soc. 1949, 2, 37-39.

57. Oostenbrink, M. A note on Paratylenchus in the Netherlands with the description of P. goodeyi n. sp. (Nematoda, Criconematidae). Tijdschr. Plantenziekten 1953, 59, 207-216. [CrossRef]

58. Raski, D.J. Revision of the genus Paratylenchus Micoletzky, 1922 and descriptions of new species. Part II of three parts. J. Nematol. 1975, 7, 274-295. [PubMed]

59. Andrássy, I. Neue und wenig bekannte nematoden aus Jugoslawien. Ann. Hist. Nat. Musei Natl. Hung. 1959, 51, $259-275$.

60. Cobb, N.A. Notes on Paratylenchus, a genus of nemas. J. Wash. Acad. Sci. 1923, 13, $254-257$.

61. Subbotin, S.A.; Yan, G.; Kantor, M.; Handoo, Z. On the molecular identity of Paratylenchus nanus Cobb, 1923 (Nematoda: Tylenchida). J. Nematol. 2020, accepted.

62. De Coninck, L. Sur trois espèces nouvelles de nématodes libres trouvés en Belgique. Bul. Mus. Roy. D'hist. Nat. Belg. 1931, 7, 1-5.

63. Wu, L.Y. Paratylenchus veruculatus n. sp. (Criconematidae: Nematoda) from Scotland. Can. J. Zool. 1962, 40, 773-775. [CrossRef]

64. Thorne, G.; Allen, M.W. Paratylenchus hamatus n. sp, and Xiphinema index n. sp., two nematodes associated with fig roots, with a note on Paratylenchus anceps Cobb. Proc. Helm. Soc. Wash. 1950, 17, $27-35$.

65. Meldal, B.H.; Debenham, N.J.; De Ley, P.; De Ley, I.T.; Vanfleteren, J.R.; Vierstraete, A.R.; Bert, W.; Borgonie, G.; Moens, T.; Tyler, P.A.; et al. An improved molecular phylogeny of the Nematoda with special emphasis on marine taxa. Mol. Phylogenetics Evol. 2007, 42, 622-636. [CrossRef]

66. Ortiz, V.; Phelan, S.; Mullins, E. A temporal assessment of nematode community structure and diversity in the rhizosphere of cisgenic Phytophthora infestans-resistant potatoes. BMC Ecol. 2016, 16, 1-23. [CrossRef] [PubMed]

67. Háněl, L.; Brzeski, M. Paratylenchinae: Evaluation of diagnostic morpho-biometrical characters of females in the genus Paratylenchus Micoletzky, 1922 (Nematoda: Tylenchulidae). Nematology 2000, 2, 253-261. [CrossRef]

68. Hirschmann, H. Scanning electron microscopy as a tool in nematode taxonomy. In Concepts in Nematode Systematics; Stone, A.R., Platt, H.M., Kahlil, L.F., Eds.; Academic Press: New York, NY, USA, 1983; pp. 95-111.

69. Wergin, W.P. Scanning electron microscopic techniques and applications for use in nematology. In Plant Parasitic Nematodes; Zuckerman, B.M., Rhode, R.A., Eds.; Academic Press: New York, NY, USA, 1981; Volume 3, pp. 175-204.

70. Eisenback, J.D. Techniques for preparing nematodes for scanning electron microscopy. In An Advanced Treatise on Meloidogyne; Barker, K.R., Carter, C.C., Sasser, N.S., Eds.; Raleigh, State University Graphics: Raleigh, NC, USA, 1985; Volume 2, pp. 79-105.

71. Nassonova, E.; Smirnov, A.; Fahrni, J.; Pawlowski, J. Barcoding amoebae: Comparison of SSU, ITS and COI genes as tools for molecular identification of naked lobose amoebae. Protist 2010, 161, 102-115. [CrossRef] 
72. Morise, H.; Miyazaki, E.; Yoshimitsu, S.; Eki, T. Profiling nematode communities in unmanaged flowerbed and agricultural field soils in Japan by DNA barcode sequencing. PLoS ONE 2012, 7, 1-10. [CrossRef] [PubMed]

73. Armenteros, M.; Rojas-Corzo, A.; Ruiz-Abierno, A.; Derycke, S.; Backeljau, T.; Decraemer, W. Systematics and DNA barcoding of free-living marine nematodes with emphasis on tropical desmodorids using nuclear SSU rDNA and mitochondrial COI sequences. Nematology 2014, 16, 979-989. [CrossRef]

74. Kiewnick, S.; Holterman, M.; van den Elsen, S.; van Megen, H.; Frey, J.E.; Helder, J. Comparison of two short DNA barcoding loci (COI and COII) and two longer ribosomal DNA genes (SSU \& LSU rRNA) for specimen identification among quarantine root-knot nematodes (Meloidogyne spp.) and their close relatives. Eur. J. Plant Pathol. 2014, 140, 97-110.

75. Pons, J.; Barraclough, T.G.; Gomez-Zurita, J.; Cardoso, A.; Duran, D.P.; Hazell, S.; Kamoun, S.; Sumlin, W.D.; Vogler, A.P. Sequence-based species delimitation for the DNA taxonomy of undescribed insects. Syst. Biol. 2006, 55, 595-609. [CrossRef]

76. Zhang, J.; Kapli, P.; Pavlidis, P.; Stamatakis, A. A general species delimitation method with applications to phylogenetic placements. Bioinformatics 2013, 29, 2869-2876. [CrossRef] [PubMed]

77. Carstens, B.C.; Pelletier, T.A.; Reid, N.M.; Satler, J.D. How to fail at species delimitation. Mol. Ecol. 2013, 22, 4369-4383. [CrossRef]

78. Leliaert, F.; Verbruggen, H.; Vanormelingen, P.; Steen, F.; López-Bautista, J.M.; Zuccarello, G.C.; De Clerck, O. DNA-based species delimitation in algae. Eur. J. Phycol. 2014, 49, 179-196. [CrossRef]

79. Janssen, T.; Karssen, G.; Orlando, V.; Subbotin, S.; Bert, W. Molecular characterization and species delimiting of plant parasitic nematode of the genus Pratylenchus from the Penetrans group (Nematoda: Pratylenchidae). Mol. Phylogenet. Evol. 2017, 117, 30-48. [CrossRef]

80. Qing, X.; Bert, W.; Gamliel, A.; Bucki, P.; Duvrinin, S.; Alon, T.; Miyara, S.B. Phylogeography and molecular species delimitation of Pratylenchus capsici n. sp., a new root-lesion nematode in Israel on pepper (Capsicum annuum). Phytopathology 2018, 109, 847-858. [CrossRef]

81. Xu, X.; Qing, X.; Xie, J.L.; Yang, F.; Peng, Y.L.; Ji, H.L. Population structure and species delimitation of rice white tip nematode, Aphelenchoides besseyi (Nematoda: Aphelenchoididae), in China. Plant Pathol. 2020, 69, 159-167. [CrossRef]

82. Padial, J.M.; De la Riva, I. A paradigm shift in our view of species drives current trends in biological classification. Biol. Rev. 2020, 23, 1-21.

83. Prévot, V.; Jordaens, K.; Sonet, G.; Backeljau, T. Exploring Species Level Taxonomy and Species Delimitation Methods in the Facultatively Self-Fertilizing Land Snail Genus Rumina (Gastropoda: Pulmonata). PLoS ONE 2013, 8, e60736.

84. Zink, R.M.; Barrowclough, G.F. Mitochondrial DNA under siege in avian phylogeography. Mol. Ecol. 2008, 17, $2107-2121$. [CrossRef]

85. Palumbi, S.R.; Cipriano, F.; Hare, M.P. Predicting nuclear gene coalescence from mitochondrial data: The three-times rule. Evolution 2001, 55, 859-868. [CrossRef]

86. Jörger, K.M.; Norenburg, J.L.; Wilson, N.G.; Schrödl, M. Barcoding against a paradox? Combined molecular species delineations reveal multiple cryptic lineages in elusive meiofaunal sea slugs. BMC Evol. Biol. 2012, 12, 1-18. [CrossRef] [PubMed]

87. Puillandre, N.; Modica, M.V.; Zhang, Y.; Sirovich, L.; Boisselier, M.C.; Cruaud, C.; Holford, M.; Samadi, S. Large-scale species delimitation method for hyperdiverse groups. Mol. Ecol. 2012, 21, 2671-2691. [CrossRef]

88. Padial, J.M.; Miralles, A.; De la Riva, I.; Vences, M. The integrative future of taxonomy. Front. Zool. 2010, 7, 1-16. [CrossRef]

89. Whitehead, A.G.; Hemming, J.R. A comparison of some quantitative methods of extracting small vermiform nematodes from soil. Ann. Appl. Biol. 1965, 55, 25-38. [CrossRef]

90. Jenkins, W.R.B. A rapid centrifugal-flotation technique for separating nematodes from soil. Plant Dis. Rep. 1964, 48, 692.

91. Available online: https:/ / www.youtube.com/watch?v=qWITH1yB1U0\&t=2s (accessed on 15 February 2021).

92. Singh, P.R.; Nyiragatare, A.; Janssen, T.; Couvreur, M.; Decraemer, W.; Bert, W. Morphological and molecular characterisation of Pratylenchus rwandae n. sp. (Tylenchida: Pratylenchidae) associated with maize in Rwanda. Nematology 2018, 20, 781-794. [CrossRef]

93. Nunn, G.B. Nematode Molecular Evolution. An Investigation of Evolutionary Patterns among Nematodes Based Upon DNA Sequences. Ph.D. Thesis, University of Nottingham, Nottingham, UK, 1992.

94. Vrain, T.C.; Wakarchuk, D.A.; Levesque, A.C.; Hamilton, R.I. Intraspecific rDNA restriction fragment length polymorphism in the Xiphinema americanum group. Fundam. Appl. Nematol. 1992, 15, 563-573.

95. Curran, J.; Driver, F.; Ballard, J.W.O.; Milner, R.J. Phylogeny of Metarhizium: Analysis of ribosomal DNA sequence data. Mycol. Res. 1994, 98, 547-552. [CrossRef]

96. Mayer, W.E.; Herrmann, M.; Sommer, R.J. Phylogeny of the nematode genus Pristionchus and implications for biodiversity, biogeography and the evolution of hermaphroditism. BMC Evol. Biol. 2007, 7, 1-13. [CrossRef] [PubMed]

97. Singh, P.R.; Couvreur, M.; Decraemer, W.; Bert, W. Survey of slug-parasitic nematodes in East and West Flanders, Belgium and description of Angiostoma gandavensis n. sp. (Nematoda: Angiostomidae) from arionid slugs. J. Helminthol. 2019, 94, 1-11. [CrossRef]

98. Tanha Maafi, Z.; Subbotin, S.A.; Moens, M. Molecular identification of cyst-forming nematodes (Heteroderidae) from Iran and a phylogeny based on the ITS sequences of rDNA. Nematology 2003, 5, 99-111. [CrossRef]

99. Bowles, J.; Blair, D.; McManus, D.P. Genetic variants within the genus Echinococcus identified by mitochondrial DNA sequencing. Mol. Biochem. Parasitol. 1992, 54, 165-173. [CrossRef] 
100. Powers, T.O.; Bernard, E.C.; Harris, T.; Higgins, R.; Olson, M.; Lodema, M.; Mullin, P.; Sutton, L.; Powers, K.S. COI haplotype groups in Mesocriconema (Nematoda: Criconematidae) and their morphospecies associations. Zootaxa 2014, 3827, 101-146. [CrossRef]

101. Singh, P.R.; Karssen, G.; Couvreur, M.; Bert, W. Morphological and molecular characterization of Heterodera dunensis n. sp.(Nematoda: Heteroderidae) from Gran Canaria, Canary Islands. J. Nematol. 2020, 52, 1-14.

102. Available online: https://dna.macrogen.com (accessed on 30 December 2020).

103. Available online: https://www.genewiz.com (accessed on 15 December 2020).

104. Thompson, J.D.; Gibson, T.J.; Plewniak, F.; Jeanmougin, F.; Higgins, D.G. The CLUSTAL_X windows interface: Flexible strategies for multiple sequence alignment aided by quality analysis tools. Nucleic Acids Res. 1997, 25, 4876-4882. [CrossRef]

105. Subbotin, S.A.; Sturhan, D.; Adams, B.J.; Powers, T.O.; Mullin, P.G.; Chizhov, V.N.; Vovlas, N.; Baldwin, J.G. Molecular phylogeny of the order tylenchida: Analysis of nuclear ribosomal RNA genes. J. Nematol. 2006, 38, 296.

106. Huelsenbeck, J.P.; Ronquist, F. MRBAYES: Bayesian inference of phylogenetic trees. Bioinformatics 2001, 17, 754-755. [CrossRef] [PubMed]

107. Swofford, D.L.; Sullivan, J. Phylogeny inference based on parsimony and other methods using PAUP* Practice. In The Phylogenetic Handbook, a Practical Approach to DNA and Protein Phylogeny; Salemi, M., Vandamme, A.M., Eds.; Cambridge University Press: Cambridge, UK, 2003; pp. 182-206.

108. Bandelt, H.J.; Forster, P.; Röhl, A. Median-joining networks for inferring intraspecific phylogenies. Mol. Biol. Evol. 1999, 16, 37-48. [CrossRef]

109. Drummond, A.J.; Suchard, M.A.; Xie, D.; Rambaut, A. Bayesian phylogenetics with BEAUti and the BEAST 1.7. Mol. Biol. Evol. 2012, 29, 1969-1973. [CrossRef] [PubMed]

110. Available online: https://species.h-its.org (accessed on 10 December 2020).

111. Available online: https://species.h-its.org/gmyc (accessed on 10 December 2020). 